University of Louisville

ThinkIR: The University of Louisville's Institutional Repository

Electronic Theses and Dissertations

6-1997

\title{
Calibration methodology and multiple tip structure by scanning tunneling microscopy.
}

Michael Stephen Gossman

University of Louisville

Follow this and additional works at: https://ir.library.louisville.edu/etd

Part of the Physics Commons

\section{Recommended Citation}

Gossman, Michael Stephen, "Calibration methodology and multiple tip structure by scanning tunneling microscopy." (1997). Electronic Theses and Dissertations. Paper 2738.

https://doi.org/10.18297/etd/2738

This Master's Thesis is brought to you for free and open access by ThinkIR: The University of Louisville's Institutional Repository. It has been accepted for inclusion in Electronic Theses and Dissertations by an authorized administrator of ThinkIR: The University of Louisville's Institutional Repository. This title appears here courtesy of the author, who has retained all other copyrights. For more information, please contact thinkir@louisville.edu. 


\title{
CALIBRATION METHODOLOGY AND MULTIPLE TIP STRUCTURE BY SCANNING TUNNELING MICROSCOPY
}

A Study Of Calibration Techniques And Multiple Tip Structure Based On Topographic Images Of Carbon From A Scanning Tunneling Microscope

By

Michael Stephen Gossman

B.S., University of Louisville, 1995

\begin{abstract}
A Thesis
Submitted to the Faculty of the Graduate School of the University of Louisville in Partial Fulfillment of the Requirements

for the Degree of
\end{abstract}

Master of Science

Department of Physics

University of Louisville

Louisville, Kentucky

June 1997 


\section{CALIBRATION METHODOLOGY AND MULTIPLE TIP STRUCTURE BY SCANNING TUNNELING MICROSCOPY}

A Study Of Calibration Techniques And Multiple Tip Structure Based On Topographic Images Of Carbon From A Scanning Tunneling Microscope

By

Michael Stephen Gossman

B.S., University of Louisville, 1995

A Thesis Approved on

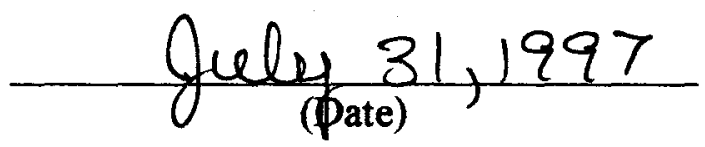

by the following Reading Committee:

(Thesis Director) 


\begin{abstract}
The scanning tunneling microscope has been in commercial use just over a decade. Due to its Quantum Mechanical nature, experiments in this area have been widely publicized to the scientific community. The apparatus is steadily growing easier to use, though fundamental problems are still present.

In the first chapter, an address is given on the modern theory of tunneling to rationalize the conclusions Physicists made long ago about this phenomenon. The expanded theory generalizes the mathematical motivation for attempting such unique experiments and serves as a lead-in for the major components of the apparatus. Many texts do not quantize what the purpose is for each unit in the STM system, nor do they illustrate the process of preparation for probe shaping. It is the objective of the second and third chapters to detail this information.
\end{abstract}

With as much as researchers know about the STM, there still remains an abundance of imaging problems which can develop. A study of graphite will be given in the fourth chapter dealing with one such issue - incorrect topograph scaling. Calibration techniques are provided to effectively determine the scaling needed for images using the horizontal and verticle lattice constants. Steps which appear as layering transitions are used for vertical calibration while B-atom spacing is used for horizontal calibration.

Due to the conductive nature of the probe, particulate fragments are more likely to attach to the apex of the probe than to uncharged material in the system. Thus, since the probe's shape is the most important item to control in STM experiments, atomic resolutions of topographs are often in danger. One of many consequences of having two 
atoms on the apex of the probe is a duplication of sample structures on the topographic image. In the fifth chapter, it is proposed that one could determine the structure of a double tip if an imperfection existed on the sample surface for reference. The imperfection used for this study is a hole created by prior tip contact with the graphite sample. The original and duplicate images of the cavity are used to determine this double tip structure. 


\section{ACKNOWLEDGEMENTS}

The author greatfully acknowledges Dr. P. J. Ouseph for his assistance and educational guidance in the experiments undertaken. He has provided creative insight for planning and unertaking these experiments. In the eyes of this student, P. J.'s wisdom and abilities have transpired his appearance to be that of an educational mentor.

The author also wishes to express his thanks to Dr. David W. Taylor from the Indiana University Southeast Biology Department for the use of photographic equipment. Pictures of the tungsten wires produced by the powerful Olympus microscope and camera have brought forth observable understanding for probe shaping. 


\section{TABLE OF CONTENTS}

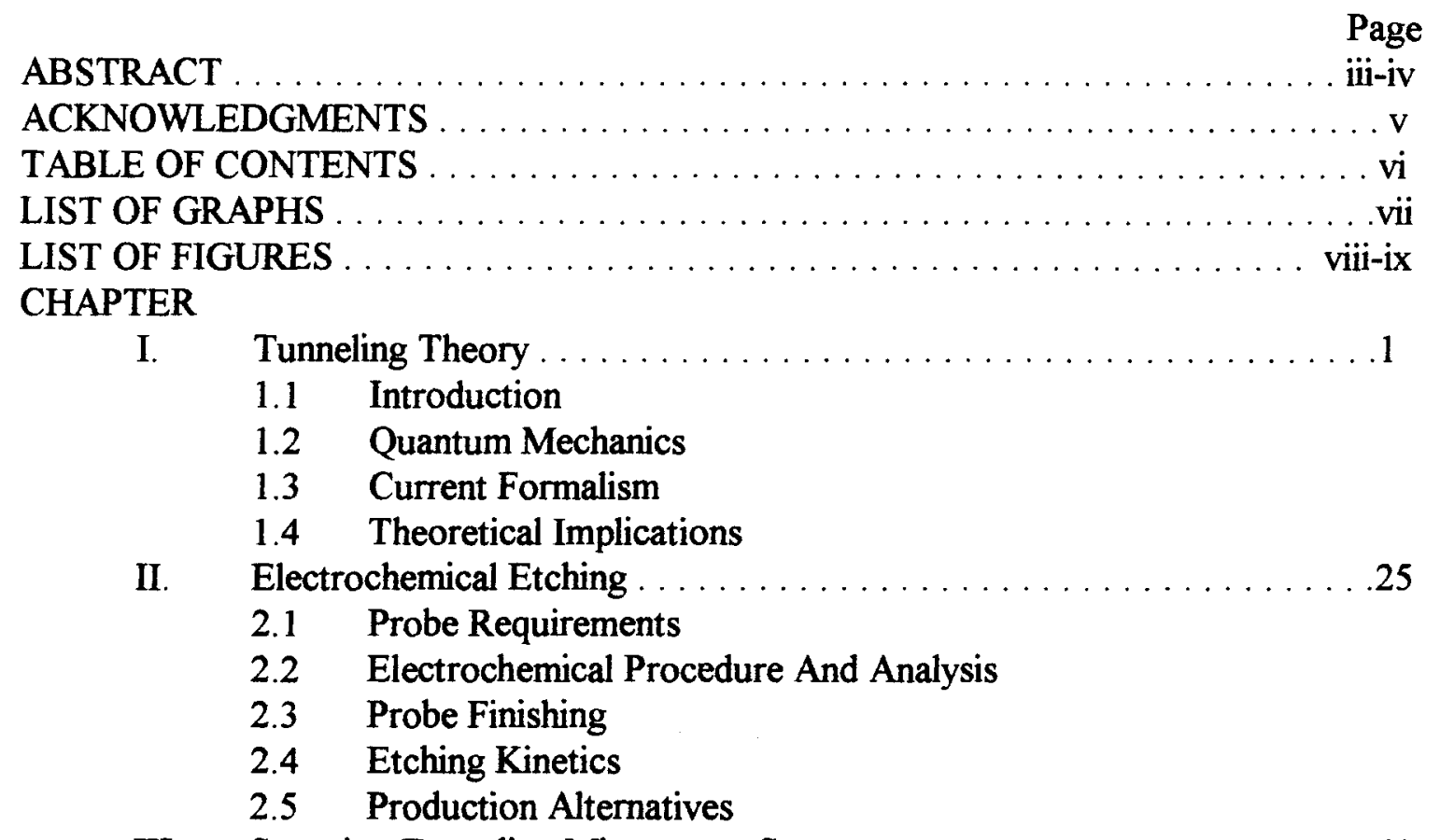

III. Scanning Tunneling Microscope System . . . . . . . . . . . . . . 41

3.1 Microscope Apparatus

3.2 Sample Lift Mechanism

3.3 Comparator And Driver Card

3.4 Function Generator

3.5 Pre-Amplifier

3.6 Oscilloscope

3.7 Piezoelectric Tube

3.8 Model 100 STM

3.9 Software

IV. Calibration Methodology : Experiment I . . . . . . . . . . . . . . .59

4.1 Sample Preparation

4.2 Calibration Hypothesis

4.3 Calibration Review

4.4 Calibration Experiment

V. Multiple Tip Structure : Experiment II . . . . . . . . . . . . . . . . . 79

5.1 Structure Hypothesis

5.2 Structure Review

5.3 Structure Experiment

VI. Results And Discussion . . . . . . . . . . . . . . . . . . . . . . 101

6.1 Analysis Conclusions

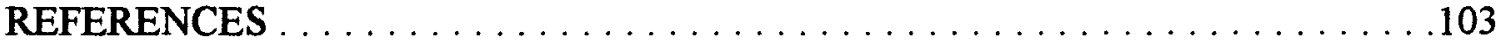

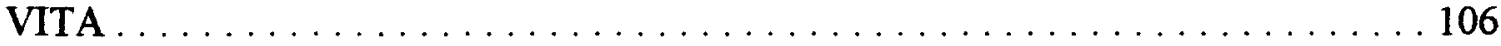




\section{LIST OF GRAPHS}

Page

GRAPH 1. Wet-length versus time plot $\ldots \ldots \ldots \ldots \ldots \ldots \ldots \ldots \ldots \ldots$

GRAPH 2. Wet-length versus current plot $\ldots \ldots \ldots \ldots \ldots \ldots \ldots \ldots \ldots$

GRAPH 3. Correction factor versus step height plot $\ldots \ldots \ldots \ldots \ldots$ 


\section{LIST OF FIGURES}

Page

FIGURE 1. Theoretical electron tunneling particle path and wave path $\ldots \ldots \ldots . .3$

FIGURE $2 . \quad$ Fermi energy state diagram $\ldots \ldots \ldots \ldots \ldots \ldots$

FIGURE 3. Electrochemical etching kinetics $\ldots \ldots \ldots \ldots \ldots \ldots \ldots \ldots \ldots$

FIGURE 4. Oxidation-reduction appararus connections $\ldots \ldots \ldots \ldots$

FIGURE 5. An etched tungsten tip beside a $0.5 \mathrm{~mm}$ scaled ruler $\ldots \ldots \ldots \ldots . \ldots 34$

FIGURE 6. An etched tungsten tip beside an unetched wire $\ldots \ldots \ldots \ldots$

FIGURE 7. Scanning tunneling tip apparatus $\ldots \ldots \ldots \ldots \ldots \ldots \ldots \ldots \ldots$

FIGURE 8. Scanning tunneling sample apparatus. . . . . . . . . . . 44

FIGURE 9. Scanning tunneling microscope system $\ldots \ldots \ldots \ldots \ldots \ldots \ldots$

FIGURE 10. Piezoelectric lead-zirconate-titanate ceramic $\ldots \ldots \ldots \ldots \ldots \ldots \ldots$

FIGURE 11. 3D carbon-graphite structure diagram $\ldots \ldots \ldots \ldots \ldots \ldots \ldots$

FIGURE 12. Uncalibrated cross section in the $\mathbf{x}-\mathbf{y}$ plane $\ldots \ldots \ldots \ldots \ldots \ldots$

FIGURE 13. Calibrated cross section in the $\mathbf{x}-\mathbf{y}$ plane $\ldots \ldots \ldots \ldots \ldots$



FIGURE 15. Uncalibrated $2 \mathrm{D}$ triple step I cross section $\ldots \ldots \ldots \ldots \ldots \ldots$

FIGURE 16. Uncalibrated $2 \mathrm{D}$ triple step II cross section $\ldots \ldots \ldots \ldots \ldots \ldots$

FIGURE 17. Uncalibrated $3 D$ triple step $1 \ldots \ldots \ldots \ldots \ldots \ldots$

FIGURE 18. 2D $1313 \AA$ wide crash hole sight first seen $\ldots \ldots \ldots \ldots \ldots$. . . 87

FIGURE 19. 3D $1313 \AA$ wide crash hole sight first seen $\ldots \ldots \ldots \ldots \ldots$

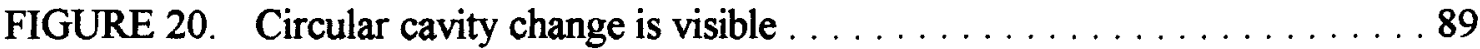




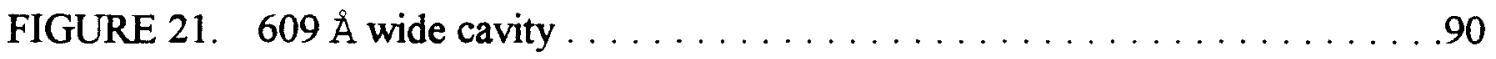

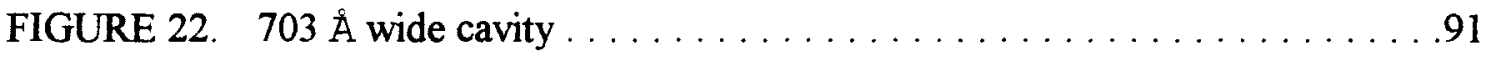

FIGURE 23. $656 \AA$ wide initial hole with a $469 \AA$ wide shadow . . . . . . . . 92

FIGURE 24. $563 \AA$ wide initial hole with a $591 \AA$ wide shadow . . . . . . . . . 94

FIGURE 25. Shadow hole has transformed almost completely . . . . . . . . . 96

FIGURE 26. Transformation depiction for the double-tip $\ldots \ldots \ldots \ldots \ldots \ldots$ 


\section{CHAPTER I \\ TUNNELING THEORY}

\subsection{Introduction}

The first use of a scanning tunneling microscope (STM) was celebrated in 1982, by its developers Gerd Binnig and Heinrich Rohrer of the IBM Corporation. It was evident to the 1986 Nobel Prize committee of the Royal Sweedish Academy of Sciences that the achievement would advance science and the two Zurich Laboratory researchers were awarded the most prestigious Physics award of that year. Binnig and Rohrer were not the only scientists to receive the honors in 1986. Ernst Ruska of Berlin, Germany also received the Nobel Prize in Physics for his earlier efforts in producing the first ever electron microscope. These events accelerated the further study and systemization of the STM to produce superior atomic scale images. The use of the scanning tunneling microscope has brought tremendous success in surface analysis for fifteen years. ${ }^{1}$

Modern adaptable software helps in producing excellent views of two and three dimensional topographs of atoms in their structural environment. Real time concepts allow one to understand the nature of electronic properties of atoms as well as certain reactions they have with neighboring atoms. Using a STM to generate images of atomic structures have also been used to investigate how biological molecules are built.

At present, one of the most interesting new applications for the use of a scanning tunneling microscope is in molecular physics. Fascinating STM images of DNA confirm its helicity. ${ }^{2}$ These reproducible results have enhanced the current knowledge regarding 
genetic structure. The biological images provide insight on segment grouping as well as protein and enzyme locations.

Recurring surface studies are also being done on the conducting surfaces of silicon $(\mathrm{Si})$, carbon $(\mathrm{C})$, gold $(\mathrm{Au})$, platinum $(\mathrm{Pt})$, and germanium $(\mathrm{Ge})$ to name a few. Since the introduction of the STM, atomic symmetry, steps on crystal surfaces, and dislocations have been widely investigated.

\subsection{Quantum Mechanics}

Tunneling is a phenomenon known to be theoretically possible in the view of Quantum Mechanics. An electron is said to have a probability of penetrating a potential barrier even if the barrier potential has a magnitude greater than the electron's incident energy. Classical Mechanics does not permit such an occurrence. The old theory implies that the potential energy from the barrier opposes the kinetic energy of the particle such that it reflects from the barrier's position totally. FIGURE 1 shows a typical square barrier for this problem.

If a barrier exists with a potential height $V_{o}$, and an electron with incident kinetic energy $E<V_{o}$ is directed upon it, the electron cannot penetrate the barrier and thus should not be present beyond the barrier. Classically, such an event would indicate that the particle is allowed a negative kinetic energy. Experimentally with a STM, this phenomenon has occurred.

Quantum Mechanics proposes that there exists a duality to the behavior of the electron in that it behaves not only as a particle but also as a wave. The theoretical wave 

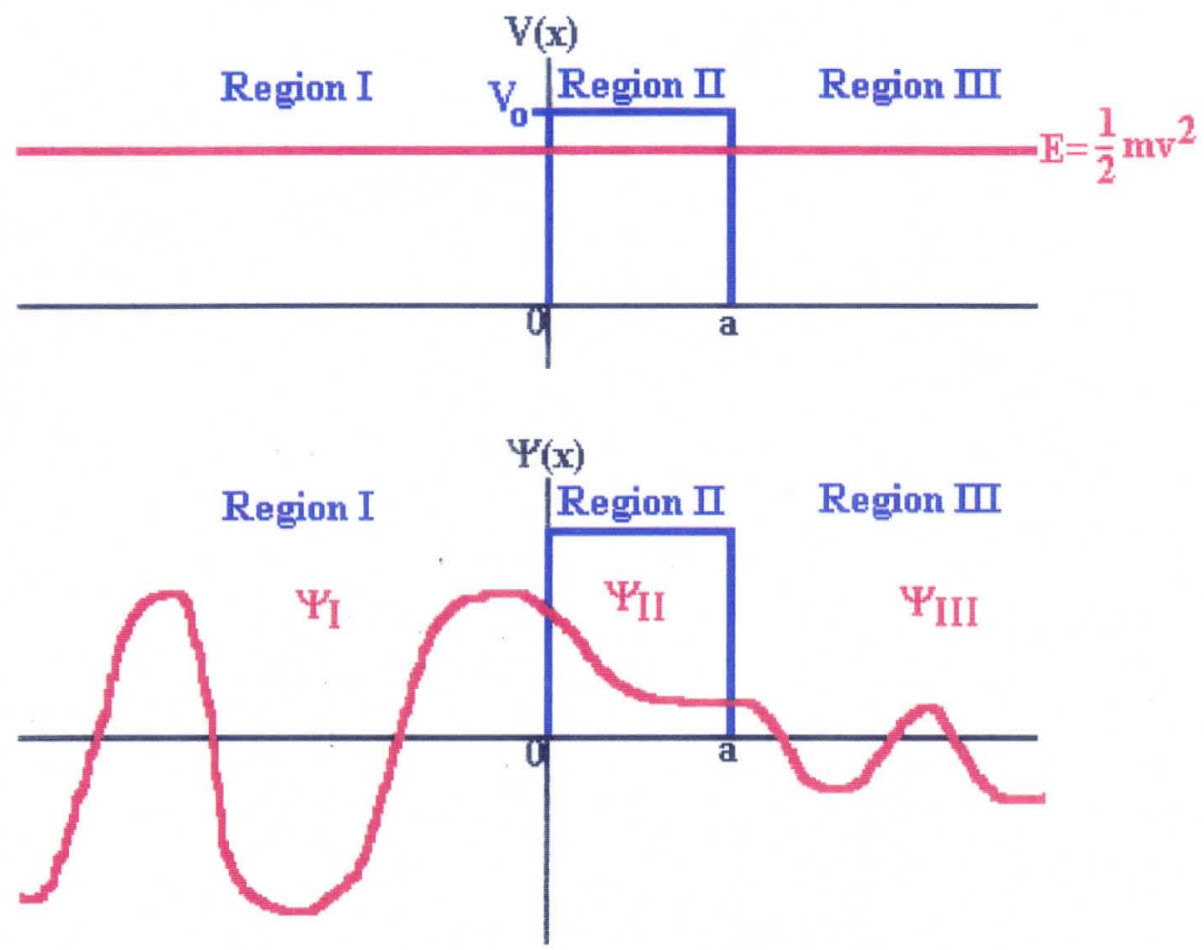

FIGURE 1. Theoretical electron tunneling particle path and wave path 
path is also shown in FIGURE 1. This novel modern theory can be easily understood by utilizing aspects of wave motion and equations which the electron wave must obey.

An eigenfunction associated with the free electron must be a solution to the TimeIndependent Schrödinger equation. Furthermore, it must satisfy the equation for all of the different regions of position involved. Using the general form of the wave equation, three regions give three eigenfunctions that satisfy this criteria.

$$
\text { Time-Independent Schrödinger Equation : } \quad \frac{-h^{2} d^{2} \Psi(x)}{8 \pi^{2} m d^{2} x}+V(x) \Psi(x)=E \Psi(x)
$$

$$
\begin{aligned}
& \text { Region I : } \quad \mathrm{x}<0 \quad \mathrm{k}_{\mathrm{I}}^{2}=\underline{8 \pi^{2} \mathrm{mE}} \\
& \frac{-h^{2} d^{2} \Psi_{I}}{8 \pi^{2} m d^{2} x}=E \Psi_{I} \quad \Psi_{I}=A \exp \left(i_{I} x\right)+B \exp \left(-\mathrm{ik}_{\mathrm{I}} \mathrm{x}\right)
\end{aligned}
$$

$$
\begin{aligned}
& \text { Region II : } \quad 0<x<a \quad \quad k_{\Pi}^{2}=\underline{8 \pi^{2} m\left(V_{0}-E\right)} \\
& \frac{-h^{2}}{8 \pi^{2}{ }^{2} \Psi^{2} \Psi_{I}}+V_{0} \Psi_{I I}=E \Psi_{I I} \quad \Psi_{I I}=C \exp \left(-k_{I I} x\right)+D \exp \left(k_{I I} x\right)
\end{aligned}
$$

$$
\begin{aligned}
& \text { Region III : } \quad a<x \quad k_{I}^{2}=\frac{8 \pi^{2}}{h^{2}} \frac{m E}{} \\
& \frac{-h^{2}}{8 \pi^{2} \mathrm{~d}^{2} \Psi^{2} \mathrm{x}}=E \Psi_{\mathrm{II}} \quad \quad \Psi_{\mathrm{II}}=\operatorname{Eexp}\left(\mathrm{ik}_{\mathrm{I}} \mathrm{x}\right)
\end{aligned}
$$

There are two terms in each of the three eigenfunctions except for the outgoing wave in region III. This is a characteristic of the direction that the electron propagates. A positive exponential indicates that the electron is moving in the $+\mathbf{x}$ direction. A negative exponential indicates that the electron is moving in the $-\mathbf{x}$ direction. These are the 
transmittance and reflectance terms true for the electron if it is in region $I$. After the electron has tunneled through the barrier, there is no potential to change its momentum. Therefore, there can be no reflective term of the form $F \exp \left(-i k_{1} x\right)$ for $\Psi_{I I I}$. In region II the exponentials differ by a factor of $(-1)^{1 / 2}$. This is true if the value of $V_{o}$ is less than $E$, since it would infer that the value of $k_{l l}$ be imaginary.

Along with the fact that the eigenfunctions must be solutions to the wave equation, the solutions must also be well-behaved. If the solutions are continuous and smooth across the boundary of the barrier, then each $\Psi(x)$ for the free particle must obey certain continuity equations. These relations are easily seen.

$$
\begin{aligned}
& \text { Continuity Equations : } \quad \Psi_{\mathrm{I}}(\mathrm{x}=0)=\Psi_{\mathrm{II}}(\mathrm{x}=0) \quad \frac{\mathrm{d} \Psi_{\mathrm{I}}(\mathrm{x}=0)}{\mathrm{dx}}=\frac{\mathrm{d} \Psi_{\mathrm{II}}(\mathrm{x}=0)}{\mathrm{dx}} \\
& \Psi_{\text {II }}(x=a)=\Psi_{\text {III }}(x=a) \quad \frac{d \Psi_{I}(x=a)}{d x}=\frac{d \Psi_{m}}{d x}(x=a)
\end{aligned}
$$

Substituting the correct wave functions into the continuity equations yield the following results:

$$
\begin{aligned}
& A \exp \left(i k_{I} x\right)+B \exp \left(-i k_{I} x\right)=C \exp \left(-k_{I} x\right)+D \exp \left(k_{I} x\right) \\
& A \exp \left(i k_{I} 0\right)+B \exp \left(-i k_{I} 0\right)=C \exp \left(-k_{I} 0\right)+D \exp \left(k_{I} 0\right) \\
& A+B=C+D \\
& A i k_{I}\left(i k_{I} x\right)-B i k_{I} \exp \left(-i k_{I} x\right)=-C k_{I} \exp \left(-k_{I} x\right)+D k_{I} \exp \left(k_{I} x\right) \\
& A i k_{I}\left(i k_{I} 0\right)-B i k_{I} \exp \left(-i k_{I} 0\right)=-C k_{I} \exp \left(-k_{I} 0\right)+D k_{I} \exp \left(k_{I} 0\right)
\end{aligned}
$$




$$
\begin{aligned}
& \mathrm{Aik}_{\mathrm{I}}-\mathrm{Bik}_{\mathrm{I}}=-\mathrm{Ck}_{\text {II }}+\mathrm{Dk}_{\text {II }} \\
& A-B=-\frac{C k_{I I}}{i k_{I}}+\frac{D k_{I I}}{i k_{I}} \\
& \mathrm{~A}-\mathrm{B}=\underset{\mathrm{k}_{\mathrm{I}}}{\mathrm{Ci} \underline{\mathrm{k}}_{\mathrm{II}}}-\underset{\mathrm{k}_{\mathbf{I}}}{\mathrm{Dik}} \\
& C \exp \left(-k_{\mathbb{I}} \mathrm{x}\right)+\operatorname{Dexp}\left(k_{\mathbb{I}} \mathrm{X}\right)=\operatorname{Eexp}\left(\mathrm{ik}_{\mathbf{I}} \mathrm{x}\right) \\
& C \exp \left(-k_{\mathbb{I}} a\right)+\operatorname{Dexp}\left(k_{\mathbb{I}} a\right)=\operatorname{Eexp}\left(i_{\mathbb{I}} a\right) \\
& -C k_{\mathbb{I}} \exp \left(-k_{\mathbb{I}} \mathrm{x}\right)+D k_{\mathbb{I}} \exp \left(k_{\mathbb{I}} \mathrm{x}\right)=E i k_{\mathbb{I}} \exp \left(i k_{\mathbb{I}} \mathrm{x}\right) \\
& -C \exp \left(-k_{\Pi} a\right)+D \exp \left(k_{\Pi} a\right)=\underset{k_{\mathbb{I I}}}{E i_{\mathbf{I}}} \exp \left(i k_{\boldsymbol{I}} a\right)
\end{aligned}
$$

The constants represent probability densities of the wave relative to each region the electron propagates. The wave amplitude constant $A$ represents the square root of the density of the electron moving from left to right and incident on the barrier in region $I$. The square root of the density of the wave moving from left to right, away from the barrier in region $I I I$, is given by the amplitude constant $E$. If one were to get the conjugate magnitude of these two densities and take their ratio, the result would be a probability flux of barrier transmission. For simplicity, the transmission coefficient is denoted $T$, where $T=E^{*} E / A^{*} A$. Using the four equations previously found, it is now possible to formulate an acceptable approximation to the character of the tunneling phenomenon. The form $E / A$ can be found by eliminating the variables $B, C$, and $D$ between the four equations. By solving equation (3) for $D \exp \left(k_{I} a\right)$ and substituting it into equation (4), one may achieve a new equation with $C$ and $E$ variables. 


$$
\begin{aligned}
& \operatorname{Cexp}\left(-k_{11} a\right)+D \exp \left(k_{\text {II }} a\right)=\operatorname{Eexp}\left(i k_{1} a\right) \\
& \operatorname{Dexp}\left(k_{\mathbb{I}} a\right)=\operatorname{Eexp}\left(i k_{I} a\right)-C \exp \left(-k_{\mathbb{I}} a\right) \\
& -C \exp \left(-k_{\Pi} a\right)+D \exp \left(k_{\Pi} a\right)=\frac{E i k_{I}}{k_{n}} \exp \left(i k_{I} a\right) \\
& -\exp \left(-k_{\text {II }} a\right)+\left[\operatorname{Eexp}\left(i k_{1} a\right)-\operatorname{Cexp}\left(-k_{\text {II }} a\right)\right] \exp \left(k_{I I} x\right)=\underset{k_{I}}{E i_{1}} \exp \left(i k_{I} a\right) \\
& -2 C \exp \left(-k_{\Pi} a\right)=E\left(\underline{i k}_{I}-1\right) \exp \left(i k_{I} a\right) \\
& k_{\text {II }} \\
& \mathrm{C}=\underline{\mathrm{E}}\left(\underline{\mathrm{ik}}_{\mathrm{I}}-1\right) \exp \left(\mathrm{ik}_{\mathrm{I}} \mathrm{a}\right) \exp \left(\mathrm{k}_{\mathrm{I}} \mathrm{a}\right) \\
& -2 k_{\text {II }} \\
& \mathrm{C}=\underline{\mathrm{E}}\left(1-\underline{\mathbf{i k}_{\mathrm{I}}}\right) \exp \left(\mathrm{ik}_{\mathrm{I}} \mathrm{a}\right) \exp \left(\mathrm{k}_{\mathrm{I}} \mathrm{a}\right) \\
& 2 \quad \mathrm{k}_{\mathrm{II}}
\end{aligned}
$$

Solving equation (1) for $B$ and substituting it into equation (2) yields an equation which relates only the $D$ and $E$ variables.

$$
\begin{aligned}
& A+B=C+D \\
& B=C+D-A \\
& A-B=\underline{i k_{\Pi} C}-\frac{i k_{\Pi} D}{k_{I}} \\
& A-[C+D-A]=\frac{i k_{I} C}{k_{I}}-\frac{i k_{\Pi} D}{k_{I}} \\
& 2 A=C+D+\frac{i k_{\Pi} C}{k_{I}}-\frac{i k_{\Pi} D}{k_{I}} \\
& A=\frac{C}{2}\left(1+\frac{i k_{\Pi}}{k_{I}}+\frac{D}{2}\left(1-\frac{i k_{I}}{k_{I}}\right.\right.
\end{aligned}
$$


The second term with variable $D$ is considerably less than the first term with variable $A$.

Within the length of the barrier, one could approximate equation (6) withholding only the $C$ term.

$$
\mathrm{A} \cong \frac{\mathrm{C}}{2}\left(1+\underline{\underline{\mathrm{ik}}} \underline{\mathrm{k}_{\mathrm{I}}}\right)
$$

By substituting equation (5) into equation (7), the relationship between the needed densities is evident.

$$
\begin{aligned}
& C \cong \underline{E}\left(1-\underline{i k}_{I}\right) \exp \left(i k_{I} a\right) \exp \left(k_{\Pi} a\right) \\
& 2 \quad \mathrm{k}_{\text {II }} \\
& \mathrm{A} \cong \underline{\mathrm{C}}\left(1+\underline{\mathrm{ik}_{\mathrm{I}}}\right) \\
& A \cong \frac{1}{2}\left[\underline{E}\left(1-\underline{\mathrm{ik}_{\mathrm{I}}}\right) \exp \left(\mathrm{ik}_{\mathrm{I}} \mathrm{a}\right) \exp \left(\mathrm{k}_{\mathrm{I}} \mathrm{a}\right)\right]\left(1+\underline{\mathrm{k}_{\mathrm{II}}}\right) \\
& A \cong \underset{4}{E}\left(1-\underline{i k_{I}}\right)\left(1+\underline{i k_{\mathbb{I}}}\right) \exp \left(i_{k_{I}} a\right) \exp \left(k_{\text {I }} a\right)
\end{aligned}
$$

Multiplying both sides of the equation by its complex conjugate allows for the transmission coefficient to be readily obtained.

$$
\begin{aligned}
& A^{*} A \cong \underline{E} \underline{E}^{*}\left(1-\underline{i k_{I}}\right)\left(1+\underline{i k_{I}}\right)\left(1+\underline{i k_{\Pi}}\right)\left(1-\underline{i k_{\Pi}}\right) \exp \left(i k_{I_{I}} a\right) \exp \left(-i k_{I} a\right) \exp \left(k_{\mathbb{I}} a\right) \exp \left(k_{\mathbb{I}} a\right) \\
& \begin{array}{llllll}
4 & 4 & \mathrm{k}_{\mathrm{II}} & \mathrm{k}_{\mathrm{II}} & \mathrm{k}_{\mathrm{I}} & \mathrm{k}_{\mathrm{I}}
\end{array}
\end{aligned}
$$




$$
\begin{aligned}
& A^{*} A \cong \frac{E^{*} E}{16}\left(1+\frac{i_{k_{I}}^{2}}{k_{I I}^{2}}\right)\left(1+\frac{i k_{I}^{2}}{k_{I}^{2}}\right) \exp \left(2 k_{I} a\right) \\
& \frac{E^{*} E}{A^{*} A} \cong\left[\frac{1}{16}\left(1+\frac{i k_{I}^{2}}{k_{\mathrm{II}}^{2}}\right)\left(1+\frac{i k_{\Pi}^{2}}{k_{I}^{2}}\right) \exp \left(2 k_{\mathbb{I}} a\right)\right]^{-1} \\
& T \cong \frac{16 \exp \left(-2 \mathrm{k}_{\mathrm{I}} \mathrm{a}\right)}{\left(1+\frac{\underline{\mathrm{ik}}_{\mathrm{I}}^{2}}{\mathrm{k}_{\mathrm{I}}}{ }^{2}\right)\left(1+\frac{\underline{\mathrm{k}}_{\mathrm{I}}^{2}}{\mathrm{k}_{\mathrm{I}}^{2}}\right)}
\end{aligned}
$$

The notation can be made more simple by substituting the expressions for $k_{l}$ and $k_{I I}$ into the previous equation.

$$
\begin{aligned}
& \mathrm{T} \cong \underline{16} \quad \exp \left(-2\left[8 \pi^{2} \underline{\mathrm{mE}}\right]^{1 / 2} \underline{\mathrm{a} / \mathrm{h}}\right) \\
& {\left[1+\frac{\left.8 \pi^{2} \mathrm{mE} / \mathrm{h}^{2}\right]}{8 \pi^{2} \mathrm{~m}\left(\mathrm{~V}_{\mathrm{o}}-\mathrm{E}\right) / \mathrm{h}^{2}}\left[1+\frac{8 \pi^{2}}{8 \pi^{2} \mathrm{mE}\left(\mathrm{V}_{\mathrm{o}}-\mathrm{E}\right)}\right]\right.} \\
& \mathrm{T} \cong 16 \exp \left(-2\left[8 \pi^{2} \underline{\mathrm{mE}}\right]^{1 / 2} \underline{\mathrm{a} / \mathrm{h}}\right) \\
& {\left[1+\frac{E}{\left(V_{o}-E\right)}\left[1+\left(\underline{V}_{0}-E\right)\right]\right.} \\
& T \cong 16 E\left(\frac{V_{0}-E}{V_{0}^{2}}\right) \exp \left(-2\left[8 \pi^{2} \mathrm{mE}\right]^{1 / 2} \mathrm{a} / \mathrm{h}\right)
\end{aligned}
$$

As a result, the probability of the electron passing through the barrier is not equal to zero, so tunneling through it is permissible in lieu of Quantum Mechanics. The character of the tunneling effect is exemplified by the exponential decay. The argument of the exponential is merely a function of the incident kinetic energy of the electron and the width of the potential barrier. 


$$
\mathrm{T} \propto \exp \left(-\frac{4 \pi}{h}[2 \mathrm{mE}]^{1 / 2} \mathrm{a}\right)
$$

A rough approximation of the reflection coefficient $R$ can be found by observing that the total probability of the electron reflecting or transmitting is equal to one. That is to say, $R+T=1$. This is necessary to obtain an exact solution. Since the approximation was introduced, the reflection term summed together with the transmission term found similarly would not yield an exact value of unity.

The potential barrier was not allowed to change as the electron tunneled through. The height of the potential barrier was kept constant at $V_{o}$. A square potential barrier is too general to justify a good base theory for tunneling. If the form of the exponential terms in $\Psi_{l l}$ is changed where $V_{o}$ goes to $V(x)$, then the eigenfunction would not be as generalized. The value of $k_{I I}$ then changes to a form with a position dependent potential.

$$
\mathrm{k}_{\mathrm{II}}=\frac{2 \pi[2 \mathrm{~m}(\mathrm{~V}(\mathrm{x})-\mathrm{E})]^{1 / 2}}{\mathrm{~h}}
$$

Wentzel, Kramers, and Brillouin have given an approximation known as WKB which assumes that the electronic wavefunction will not alter its wavelength much if two criteria are satisfied. First, the potential change in the barrier as the electron passes must be so small that it is negligible. Second, the magnitude of incident energy must be comparable to the potential barrier's height. If the length of the wave does not change widely with respect to position, then the partial derivative magnitude of the wavelength 
with respect to position should be much less than one. ${ }^{3}$ It would then follow that the criteria from which the WKB approximation was drawn will be easily satisfied.

$$
\begin{aligned}
& \left.\left|\frac{\partial \lambda}{\partial x}\right|=\frac{\partial}{\partial x}\left|\underline{k_{I I}}\right|=\frac{\partial}{\partial x} \frac{2 \pi h}{\mid\left\{2 \pi[2 m(V(x)-E)]^{1 / 2}\right.}\right\}\left|=\frac{h}{[2 m(V(x)-E)] \partial x} \frac{\partial}{\partial x}\right|[2 m(V(x)-E)]^{1 / 2} \mid
\end{aligned}
$$

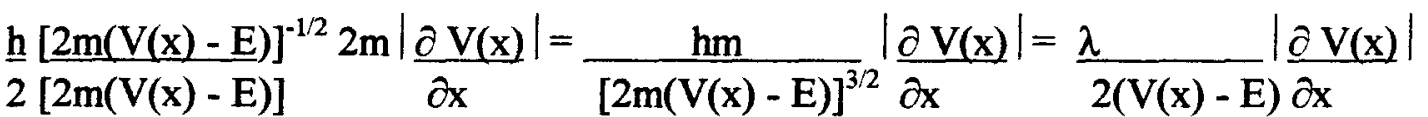

$$
\begin{aligned}
& \underline{\lambda}|\underline{\partial \mathrm{V}(\mathrm{x})}|<1 \\
& 2(V(x)-E) \partial x
\end{aligned}
$$

If the barrier length changed at any point, WKB theory speculates that the general form of the exponential argument would be known, but the form may be that of an expansion. That is to say, there may be finite corrections for the barrier pending the change in the potential. If so, the barrier length is said to be complex where the length is a complex expansion of powers of Planck's constant. ${ }^{3,4}$

$$
\begin{aligned}
& \mathrm{a} \Rightarrow \mathrm{a}_{0}(\mathrm{x})+\mathrm{ha} \mathrm{a}_{1}(\mathrm{x}) / 2 \pi+\sum_{j=2}^{j} \mathrm{~h}^{\mathrm{j}} \mathrm{a}_{\mathrm{j}}(\mathrm{x}) /(2 \pi)^{\mathrm{j}} \\
& a_{0}=h k_{\mathbb{I}} \mathrm{x} / 2 \pi \\
& a_{1}=h^{2} k_{\mathbb{I}} / 4 \pi^{2} \\
& a_{j}=h^{j+1} k_{\mathbb{I}} /(2 \pi)^{j+1}
\end{aligned}
$$

With a nearly constant potential, WKB theorists look only at the first term of the expansion. Considering the effects on only the eigenfunction which the potential has 
affected, where $\Psi_{I I}=\exp \left(-k_{I I} x\right)+\exp \left(k_{I I} x\right) \Rightarrow \exp (i 2 \pi a / h)$, it is easy to arrive at an integral form for the argument of the exponentially reducing transmission probability. The integral can be obtained by solving the differential Schrödinger equation.

$$
\begin{aligned}
& \frac{-h^{2} d^{2} \Psi(x)}{8 \pi^{2} m d^{2} x}+V(x) \Psi(x)=E \Psi(x) \\
& \frac{-h^{2}}{8 \pi^{2} m \underline{d}^{2} \Psi_{I}}+(V(x)-E) \Psi_{I I}=0 \\
& \frac{-h^{2} d^{2} \exp \left(\frac{i 2 \pi a}{h}\right)}{8 \pi^{2} m d^{2} x}+(V(x)-E) \exp \left(\frac{i 2 \pi a}{h}\right)=0 \\
& \frac{-h^{2} d}{8 \pi^{2} m d x} \frac{[i 2 \pi}{h} \exp \left(\frac{i 2 \pi a}{h}\right) \frac{d a}{d x}+(V(x)-E) \exp \left(\frac{i 2 \pi a}{h}\right)=0
\end{aligned}
$$

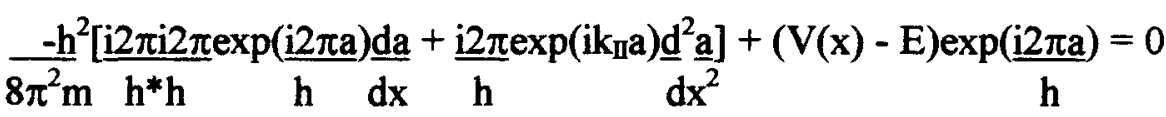

$$
\begin{aligned}
& \left\{\frac{1}{2 m}\left[\frac{|d a|^{2}}{|d x|}-\frac{i h d^{2} a}{2 \pi d x^{2}}\right]+(V(x)-E)\right\} \exp \left(\frac{i 2 \pi a)}{h}=0\right.
\end{aligned}
$$

One solution to the previous equation is therefore trivial. The expansion of variable $a$ can be substituted into the remaining expression. Keeping only the first term for constant potential renders the WKB integral approximation for the tunneling decay coefficient. Note that $d^{2} a_{0} / d x^{2}$ is exactly zero.

$$
\begin{aligned}
& \left\{\frac{1}{2 m}\left[\frac{|d a|^{2}}{|d x|}-\frac{i h d^{2}}{2 \pi d x^{2}}\right]+(V(x)-E)\right\}=0 \\
& \left.\frac{1}{2 m} \frac{\mid d a_{0}}{\mid d x}\right|^{2}=(V(x)-E)
\end{aligned}
$$




$$
\begin{aligned}
& \left.\frac{\mid \mathrm{da}_{0}}{\mid \mathrm{dx}}\right|^{2}=2 \mathrm{~m}(\mathrm{~V}(\mathrm{x})-\mathrm{E}) \\
& \mathrm{da}_{0}=[2 \mathrm{~m}(\mathrm{~V}(\mathrm{x})-\mathrm{E})]^{1 / 2} \mathrm{dx} \\
& \mathrm{a}_{0}=\int_{0}^{a}[2 \mathrm{~m}(\mathrm{~V}(\mathrm{x})-\mathrm{E})]^{1 / 2} \mathrm{dx}
\end{aligned}
$$

The final result is the widely accepted WKB approximation for the probability of transmission in a tunneling process.

$$
T(x) \equiv \exp \left(-4 \pi / h \int_{0}^{a}\left[2 m\left(V(x)-E_{x}\right)\right]^{1 / 2} d x\right)
$$

It is now left for one to utilize the theory of Quantum Mechanics to discuss the methodology behind a formulation of a tunneling current.

\subsection{Current Formalism}

Studies have found that electrical contact can be made hypothesized between two metal wires separated by atomic distances. ${ }^{5}$ It was concluded that a current flow between the separated wires is a result of a tunneling phenomenon. A tunneling current equation can be found using the previously discussed WKB approximation as a starting point. ${ }^{5}$

The probability of an electron tunneling through a potential barrier of height $V(x)$ and width $a$ was derived in Section 1.2. The system is thought to be unidirectional in $\mathbf{x}$ for the incident energy $E_{x}$. To introduce this section on current formalism, the WKB 
approximation will serve as a starting point for the fraction of electrons undergoing barrier penetration.

$$
T\left(E_{x}\right)=\exp \left[-4 \pi / h \int_{0}^{a}\left(2 m\left(V(x)-E_{x}\right)\right)^{1 / 2} d x\right]
$$

Electrons in an atom which are farther from nuclei than core electrons feel a lesser bond in the atom. Levels for electrons that participate in binding lie in continuous bands of finite separation. These particles cannot leave the atom unless they have energy sufficient enough to overcome their work function $\varphi$.

The distribution function requires the probability $f(E)$ of an electron occupying a state greater than the Fermi energy $\eta$ to be zero. Those electrons which are near the Fermi level are the only electrons which can penetrate the barrier. A conductor has its Fermi level below the top of a partially filled band. If they are in fact conducted by the positive electrode after tunneling, then the electrons will settle in unbound states of that atom just above the Fermi level. These levels are called the "conduction bands".

Given the number of electrons per unit volume $n\left(v_{x}\right) d v_{x}$ penetrating the barrier at velocities in the range of $v_{x}$ to $v_{x}+d v_{x}$ multiplied by the maximum velocities each have and the fraction of which get through, the result is the number of electrons actually passing the barrier length $N_{1}$. Again, only those electrons with sufficient kinetic energy to overcome the work function will get through. One could look at the integral either in terms of kinetic energy or velocity. The form of the two views are shown below. 


$$
\mathrm{N}=\int_{0}^{V / m} \mathrm{v}_{\mathrm{x}} \mathrm{n}\left(\mathrm{v}_{\mathrm{x}}\right) \mathrm{T}\left(\mathrm{E}_{\mathrm{x}}\right) \mathrm{dv_{ \textrm {x } }}=1 / \mathrm{m} \int_{0}^{E m} \mathrm{n}\left(\mathrm{v}_{\mathrm{x}}\right) \mathrm{T}\left(\mathrm{E}_{\mathrm{x}}\right) \mathrm{dE} \mathrm{E}_{\mathrm{x}}
$$

Since electrons follow Fermi-Dirac Statistics, the number $n\left(v_{x}\right) d v_{x}$ is found using the Fermi-Dirac distribution function $f(E)^{6}$

$$
\begin{aligned}
& n(v) d v_{x} d v_{y} d v_{z}=\left(\frac{2 m^{4}}{h^{3}}\right) f(E) d v_{x} d v_{y} d v_{z} \\
& n\left(v_{x}\right)=2 m^{4} / h^{3} \int_{-\infty}^{\infty} \int_{-\infty}^{\infty} 1 /[\exp (E-\eta)+1] d v_{y} d v_{z}=4 \pi m^{3} / h^{3} \int_{0}^{\infty} f(E) d E_{x}
\end{aligned}
$$

Substituting the number of electrons per unit volume into the equation for the number of electrons passing through, the resulting equation is a function of both the transmission fraction and the distribution function.

$$
N_{1}=4 \pi m^{2} / h^{3} \int_{0}^{E m} T\left(E_{x}\right) d E_{x} \int_{0}^{\infty} f(E) d E_{r}
$$

One may realize that the number of electrons traversing the barrier in the opposite direction takes on a similar form. The distribution function for this direction would then include the Fermi level separation energy.

$$
\mathrm{N}_{2}=4 \pi \mathrm{m}^{2} / \mathrm{h}^{3} \int_{0}^{E m} \mathrm{~T}\left(\mathrm{E}_{\mathrm{x}}\right) \mathrm{dE_{ \textrm {x } }} \int_{0}^{\infty} \mathrm{f}(\mathrm{E}+\mathrm{eV}) \mathrm{dE_{ \textrm {T } }}
$$

The total number of electrons passing the barrier is denoted by $N$. 


$$
\begin{aligned}
& N=N_{1}-N_{2}=4 \pi m^{2} / h^{3} \int_{0}^{E m} T\left(E_{x}\right) d E_{x} \int_{0}^{\infty}(f(E)-f(E+e V)) d E_{r} \\
& \xi_{1}=4 e \pi m^{2} / h^{3} \int_{0}^{\infty} f(E) d E_{r} \quad \xi_{1}=4 e \pi m^{2} / h^{3} \int_{0}^{\infty} f(E+e V) d E_{r}
\end{aligned}
$$

The tunneling current density magnitude $J$ is thus given by $J=e N$.

$$
\mathrm{J}=\int_{0}^{E m} \mathrm{~T}\left(\mathrm{E}_{\mathrm{x}}\right) \xi \mathrm{dE} \mathrm{E}_{\mathrm{x}} \quad \xi=\xi_{1}-\xi_{2}
$$

If one were to generalize the barrier such that it appears rectangular, the potential would then contain a term for the Fermi level and a term for the height of the barrier as shown in FIGURE $2^{5}$

$$
\mathrm{V}(\mathrm{x})=\eta+\varphi
$$

This is a more practical situation for a low voltage approximation. ${ }^{5}$ Since the transmission coefficient is a function of the potential, the potential change will alter the argument of the exponential. The upper limit of the barrier width integral can then be used as the approximate length.

$$
T\left(E_{x}\right)=\exp \left[-4 \pi / h \int_{0}^{a}[2 m(\eta+\varphi-E)]^{1 / 2} d x\right]
$$




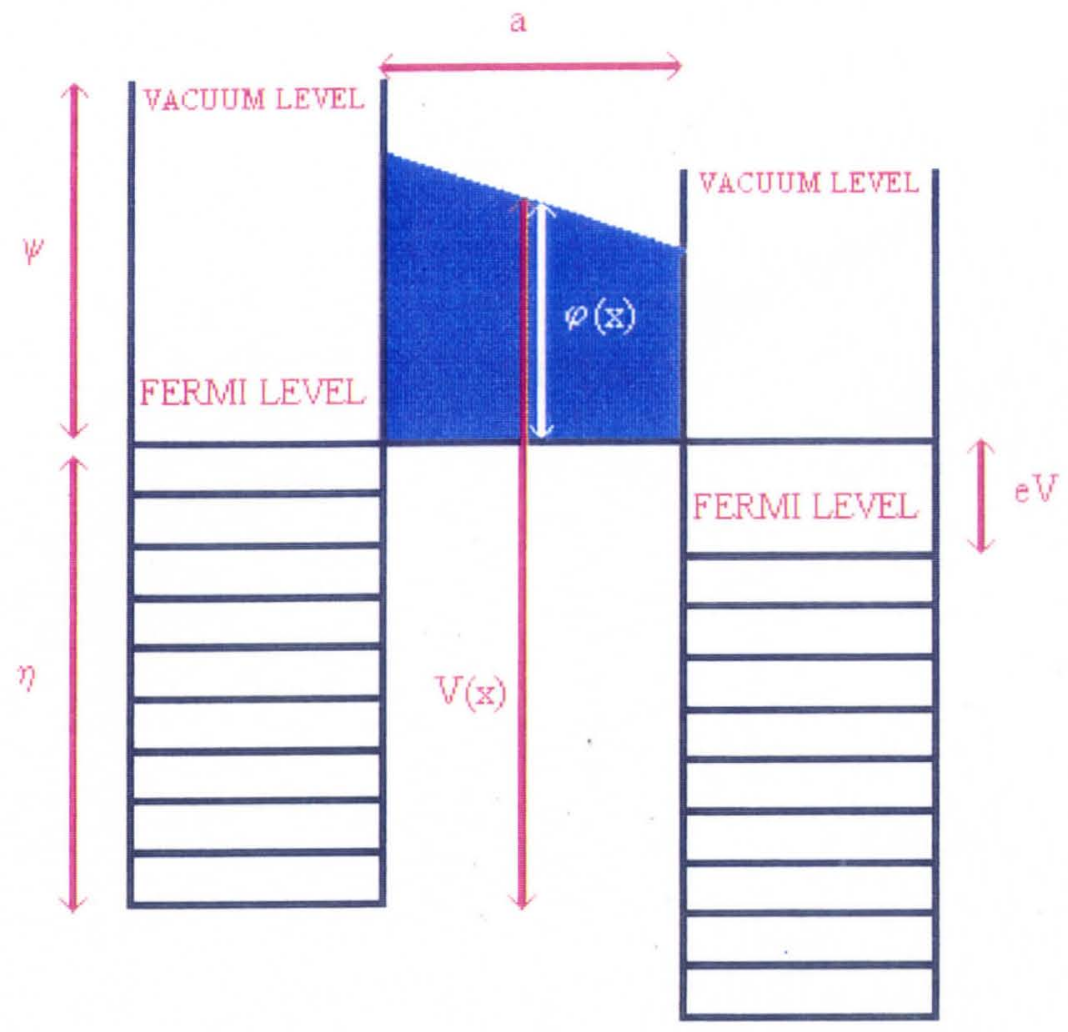

FIGURE 2. Fermi energy state diagram 


$$
\mathrm{T}\left(\mathrm{E}_{\mathrm{x}}\right) \approx \exp \left[-\frac{4 \pi(2 \mathrm{~m})^{1 / 2}}{\mathrm{a}}[\eta+\varphi-\mathrm{E}]^{1 / 2}\right]
$$

The Fermi distribution integrals take on a similar expression changes.

$$
\xi_{1}=\frac{4 \pi \mathrm{me}}{\mathrm{h}^{3}}(\eta-\mathrm{E})
$$

With $\xi=\xi_{1}-\xi_{2}$, a range can be set for the values of the Fermi expressions

$$
\begin{array}{ll}
\xi=\frac{4 \pi m e}{h^{3}}(e V) & 0<E_{x}<(\eta-e V) \\
\frac{4 \pi m e}{h^{3}}\left(\eta-E_{x}\right) & (\eta-e V)<E_{x}<\eta \\
0 & E_{x}>\eta
\end{array}
$$

With this substitution, the current density equation can be integrated.

$$
\begin{array}{r}
J=4 \pi m e / h^{3}\left[e V \int_{0}^{n-e V}\left\{\exp \left[-4 \pi / h \int_{0}^{a}\left(2 \mathrm{~m}\left(\eta+\varphi-E_{\mathrm{x}}\right)\right)^{1 / 2} \mathrm{dx}\right]\right\} \mathrm{dE} \mathrm{x}_{\mathrm{x}}+\right. \\
\left.\left(\eta-\mathrm{E}_{\mathrm{x}}\right) \int_{n-e V}^{n}\left\{\exp \left[-4 \pi / \mathrm{h} \int_{0}^{a}\left(2 \mathrm{~m}\left(\eta+\varphi-\mathrm{E}_{\mathrm{x}}\right)\right)^{1 / 2} \mathrm{dx}\right]\right\} \mathrm{dE} \mathrm{x}_{\mathrm{x}}\right]
\end{array}
$$

For simplicity, the second term is expanded by adding and subtracting the constant $\varphi$ into two terms. 


$$
\begin{gathered}
\mathrm{J}=4 \pi \mathrm{me} / \mathrm{h}^{3}\left[\mathrm{eV} \int_{0}^{n-e V}\left\{\exp \left[-4 \pi / \mathrm{h} \int_{0}^{a}\left(2 \mathrm{~m}\left(\eta+\varphi-\mathrm{E}_{\mathrm{x}}\right)\right)^{1 / 2} \mathrm{dx}\right]\right\} \mathrm{dE} \mathrm{E}_{\mathrm{x}}-\right. \\
\varphi \int_{n-e V}^{n}\left\{\exp \left[-4 \pi / \mathrm{h} \int_{0}^{a}\left(2 \mathrm{~m}\left(\eta+\varphi-\mathrm{E}_{\mathrm{x}}\right)\right)^{1 / 2} \mathrm{dx}\right]\right\} \mathrm{dE_{ \textrm {x } }}+ \\
\left.\int_{n-e V}^{n}\left(\eta+\varphi-\mathrm{E}_{\mathrm{x}}\right)\left\{\exp \left[-4 \pi / \mathrm{h} \int_{0}^{a}\left(2 \mathrm{~m}\left(\eta+\varphi-\mathrm{E}_{\mathrm{x}}\right)\right)^{1 / 2} \mathrm{dx}\right]\right\} \mathrm{dE} \mathrm{E}_{\mathrm{x}}\right]
\end{gathered}
$$

Denoting $A=\frac{4 \pi a}{h}(2 m)^{1 / 2}$, the density equation becomes more concise.

$$
\begin{aligned}
& J=\frac{8 \pi m e}{h^{3} A}\left[(e V)(\varphi+e V)^{1 / 2} \exp \left[-A(\varphi+e V)^{1 / 2}\right]+\ldots\right. \\
& \ldots+\varphi\left(A \varphi^{1 / 2}+1\right) \exp \left[-A \varphi^{1 / 2}\right]-\varphi\left(A(\varphi+e V)^{1 / 2}+1\right) \exp \left[-A(\varphi+e V)^{1 / 2}\right]+ \\
& \text { A A } \\
& \varphi^{3 / 2} \exp \left(-\mathrm{A} \varphi^{1 / 2}\right)-(\varphi+\mathrm{eV})^{3 / 2} \exp \left[-\mathrm{A}(\varphi+\mathrm{eV})^{1 / 2}\right]+\underline{3 \varphi} \exp \left(-\mathrm{A} \varphi^{1 / 2}\right)- \\
& \text { A } \\
& \left.\underline{3}(\varphi+\mathrm{eV}) \exp \left[-\mathrm{A}(\varphi+\mathrm{eV})^{1 / 2}\right]\right] \\
& \text { A }
\end{aligned}
$$

This equation has developed by assessing all terms in the first two integrals where $A(\varphi-e V)^{1 / 2}$ is much less than one. It is also the case that the third integral was approximated to two terms by the Maclaurin $\operatorname{expansion} \exp (-x)=1-x$. Some terms are negligible compared to others, and are thus discarded. This is the case for the second term in the last equation. The form of the solution for the current density from one electrode to the second is then seen when summing the seven terms. ${ }^{4}$ 


$$
J=\frac{\mathrm{e}}{2 \pi \mathrm{ha}^{2}}\left[\varphi \exp \left(-\mathrm{A} \varphi^{1 / 2}\right)-(\varphi+\mathrm{eV}) \exp \left[-\mathrm{A}(\varphi+\mathrm{eV})^{1 / 2}\right]\right]
$$

At moderate voltages where $0<V<\varphi / \mathrm{e}$, the barrier height appears to be the average of the two work functions $\phi$ lessened by the amount $\mathrm{eV} / 2$.

$$
\begin{aligned}
& \mathrm{J}=\underset{\mathrm{e} \quad\left[(\phi-\mathrm{eV} / 2) \exp \left(-\mathrm{A}(\phi-\mathrm{eV} / 2)^{1 / 2}\right)-(\phi-\mathrm{eV} / 2+\mathrm{eV}) \exp \left[-\mathrm{A}(\phi-\mathrm{eV} / 2+\mathrm{eV})^{1 / 2}\right]\right]}{2 \pi \mathrm{a}^{2}} \\
& \mathrm{~J}=\frac{\mathrm{e} \quad\left[(\phi-\mathrm{eV} / 2) \exp \left(-\mathrm{A}(\phi-\mathrm{eV} / 2)^{1 / 2}\right)-(\phi+\mathrm{eV} / 2)\right] \exp \left[-\mathrm{A}(\phi+\mathrm{eV} / 2)^{1 / 2}\right]}{2 \pi \mathrm{ha}^{2}}
\end{aligned}
$$

By inserting constants defined by the equation, the tunneling current form can be simplified for calculations.

$$
\begin{aligned}
& \left.\mathrm{J}=\underline{\mathrm{C}\left(1.6 \times 10^{-19}\right.} \underline{\mathrm{A}}^{* \mathrm{~s}}\right)\left(10^{16} \AA^{2} / \mathrm{cm}^{2}\right)\left(\underline{10}^{9} \underline{\mathrm{nA} / \mathrm{A}}\right)^{*} \ldots \\
& 2 \pi\left(4.14 \times 10^{-15} \mathrm{eV}^{*} \mathrm{~s}\right) \mathrm{a}^{2} \\
& \ldots *\left\{(\phi-\mathrm{eV} / 2) \exp \left(-\left[\underline{4 \pi\left(2 m c^{2}\right.}\right)^{1 / 2}\right](\phi-\mathrm{eV} / 2)^{1 / 2} \mathrm{a}\right)- \\
& \text { hc } \\
& \left.(\phi+\mathrm{eV} / 2) \exp \left[-\left[4 \pi\left(2 m c^{2}\right)^{1 / 2}\right](\phi+\mathrm{eV} / 2)^{1 / 2} \mathrm{a}\right]\right\} \\
& \text { hc } \\
& \mathrm{J}=\frac{2.69 \times 10^{6}}{\mathrm{~cm}^{2 *} \mathrm{a}^{2}} \underline{\mathrm{nA}} \underline{A}^{2}\left\{(\phi-\mathrm{eV} / 2)^{*} \ldots\right. \\
& \ldots{ }^{*} \exp \left[-4 \pi\left(2^{*}\left(0.511 \mathrm{MeV} / \mathrm{c}^{2}\right)^{*} \mathrm{c}^{2 *}\left(10^{6} \mathrm{eV} / \mathrm{MeV}\right)^{1 / 2}(\phi-\mathrm{eV} / 2)^{1 / 2} \underline{\mathrm{a}}\right]-\right. \\
& \left(4.14 \times 10^{-15} \mathrm{eV}^{*} \mathrm{~s}\right)\left(3.0 \times 10^{8} \mathrm{~m} / \mathrm{s}\right)\left(10^{10} \AA / \mathrm{m}\right)
\end{aligned}
$$

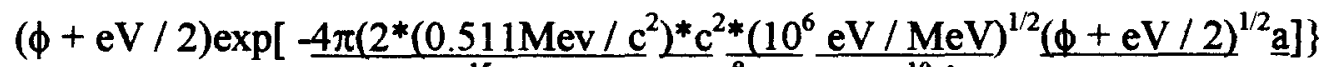

$$
\begin{aligned}
& \left(4.14 \times 10^{-15} \mathrm{eV}^{*} \mathrm{~s}\right)\left(3.0 \times 10^{8} \mathrm{~m} / \mathrm{s}\right)\left(10^{10} \AA / \mathrm{m}\right)
\end{aligned}
$$




$$
\begin{aligned}
\mathrm{J}= & \frac{2.69 \times 10^{6}}{\mathrm{~cm}^{2 *} \mathrm{a}^{2}} \frac{\mathrm{nA} * \AA^{2}}{\mathrm{eV}} \frac{(\phi-\mathrm{eV} / 2)}{\mathrm{exp}}\left[-\frac{1.025 \mathrm{a}(\phi-\mathrm{eV} / 2)^{1 / 2}}{\mathrm{eV}^{1 / 2} \AA \AA}\right]- \\
& \frac{(\phi+\mathrm{eV} / 2)}{\mathrm{eV}} \exp \left[\frac{\left.-1.025 \mathrm{a}(\phi+\mathrm{eV} / 2)^{1 / 2}\right]}{\mathrm{eV}^{1 / 2} * \AA}\right.
\end{aligned}
$$

The tunneling current can be found by multiplying the tunneling current density by the area through which the electrons are tunneling. ${ }^{5}$ The effective current $I$ between two electrodes is a function of the average of the work functions $\phi$ in electron volts, the thickness of the barrier $a$ in $\AA$, the bias voltage $V$ in volts, and the tunneling area $A$ of the conducting electrode in $\mathrm{cm}^{2}$.

$$
\begin{aligned}
\mathrm{I}= & \frac{2.69 \times 10^{6}}{\mathrm{~cm}^{2 *} \mathrm{a}^{2}} \frac{\mathrm{nA}}{*} \AA^{2} \underline{\underline{A}} \frac{\{(\phi-\mathrm{eV} / 2)}{\mathrm{eV}} \exp \left[\frac{-1.025 \mathrm{a}(\phi-\mathrm{eV} / 2}{\mathrm{eV}^{1 / 2 *} \AA}\right]- \\
& \frac{(\phi+\mathrm{eV} / 2)}{\mathrm{eV}} \exp \left[\frac{\left.\left.-1.025 \mathrm{a}(\phi+\mathrm{eV} / 2)^{1 / 2}\right]\right\}}{\mathrm{eV}^{1 / 2 *} \AA}\right.
\end{aligned}
$$

This solution is the best attempt at a closed-form approximation for tunneling current available. An exact solution for the tunneling phenomenon must be given in terms of Green's functions and in open integral-form. ${ }^{7}$ Over all, the formalism gives a formidable result. One may be able to utilize the current equation after applying correction factors to promote agreement with experimental data.

In the following experiments, a tungsten (W) wire is used to probe the surface of carbon (C) atoms in an air filled medium. The work function for tungsten is $\varphi=4.55 \mathrm{eV}$, and the work function for carbon is $\varphi=5.0 \mathrm{eV}^{8}$ The average of the two work functions is then $\phi=4.775 \mathrm{eV}$. In constant current mode, one finds the bias voltage is near $V=0.1$ 
$\mathrm{eV}$ when the tunneling current is at $I=1 \mathrm{nA}$. The separation of the two electrodes is roughly $a=7 \AA$, lending the area in this range to be roughly $A=2 \mathrm{~cm}^{2}{ }^{9}$ Thus, the most general, moderate voltage approximation has the following form where the bias and the separation are the only functional variables:

$$
\begin{gathered}
\left.I(\mathrm{a}, \mathrm{V})=\frac{5.38 \times 10^{6}}{\mathrm{a}^{2}} \AA^{2}\left\{\frac{(4.775 \text { volts }-\mathrm{V} / 2)}{\mathrm{V}}\right) \exp \left[\frac{-1.025 \mathrm{a}(4.775 \text { volts }-\mathrm{V} / 2}{\mathrm{V}^{1 / 2 * \AA}}\right)^{1 / 2}\right]- \\
\left(\frac{4.775 \text { volts }+\mathrm{V} / 2) \exp \left[\frac{\left.-1.025 \mathrm{a}(4.775 \text { volts }+\mathrm{V} / 2)^{1 / 2}\right]}{\mathrm{V}}\right] \text { nanoamperes }}{\mathrm{V}^{1 / 2 * \AA}}\right.
\end{gathered}
$$

If one wished to tabulate the tunneling current with respect to varying area, bias, work function, and separation, one could make use of the Fortran 90 program provided below. Using the resulting solution the program makes calculations easier to evaluate.

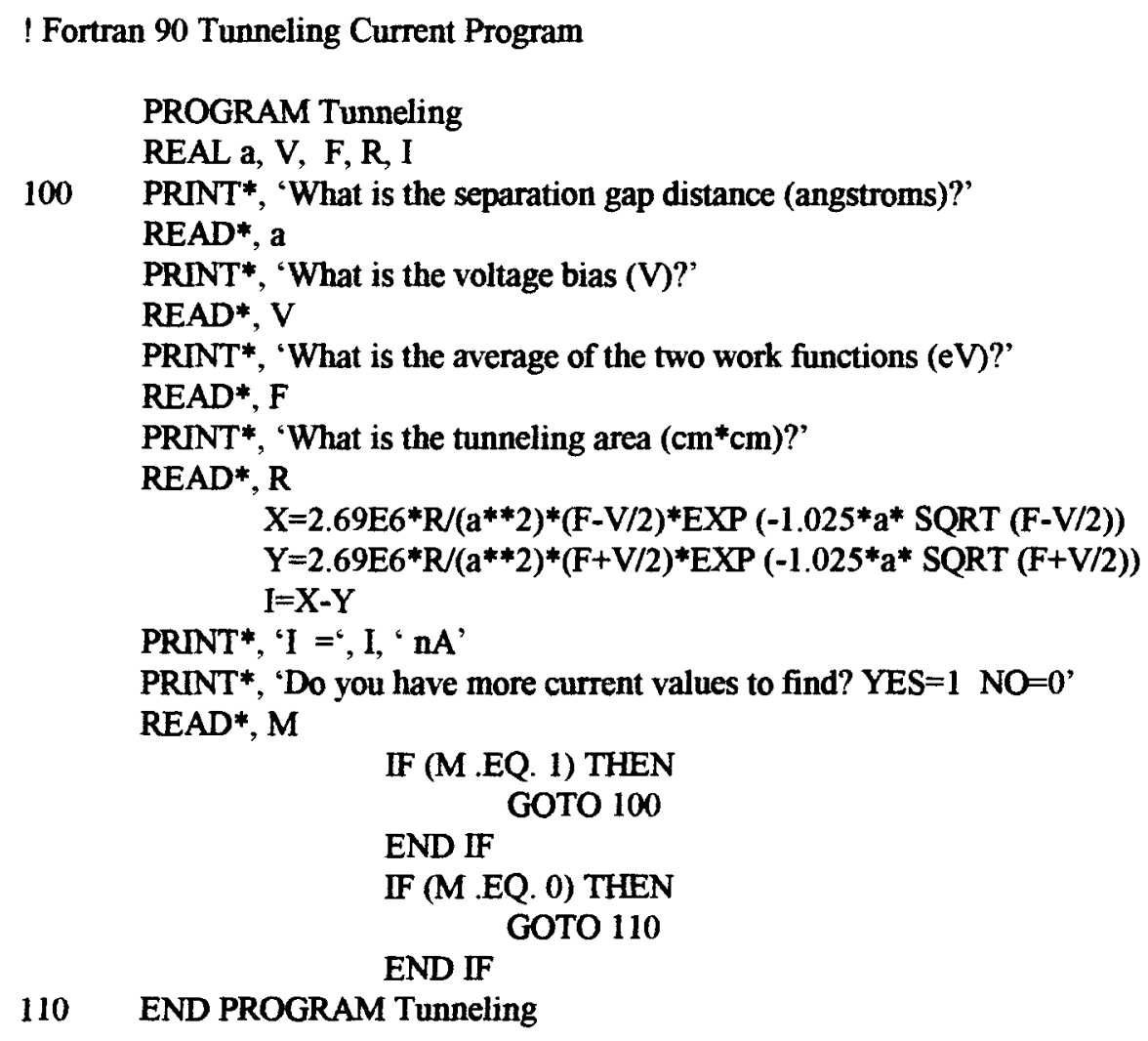




\subsection{Theoretical Implications}

It is more noticeable now that the current density equation has an exponential dependence on the square root of the mass of the tunneling particle. If the particle was a

proton, the current would be of the order $10^{-44} \mathrm{~A}$ less than the current for the electron at a barrier length of $1 \AA$. Since the exponential dependence is of the same form as the transmission coefficient, the difference implies that the proton would have a reduced probability of penetrating the barrier. In general, more massive particles have a smaller chance to tunnel through a potential barrier than do less massive particles. In order to do such an experiment with protons, one would have to use a particle accelerator. With electrons, a simple voltage in a wire would do. It is conceivably easier to perform a tunneling experiment with electrons.

It is possible to idealize the barrier model where the negative electrode has a very sharp tip, and the barrier length is the distance to some sample surface. Having such a tip would permit the tunneling emission to occur from a finite area. If the tip could be made to contain on its apex a single atom, then most of the current would flow from its Fermi level. Given a device which would allow the position of the tip to be moved in scale at an atomic level, it would then be feasible to create a current flow to different atoms of the positive sample surface. Moreover, if one could obtain readings for the exact position of the tip relative to the sample in real time, it would be possible to achieve a picture of the sample surface which relates the current to the barrier length. Using piezoelectric ceramics for atomic motion and scaling, and by using a computer for voltage and position 
interpretation, an experiment such as this can be brought about. This methodology is known to the scientific community as "scanning tunneling microscopy". 


\section{CHAPTER II}

\section{ELECTROCHEMICAL ETCHING}

\subsection{Probe Requirements}

The tip used for scanning graphite surfaces is made of polycrystalline tungsten (W) wire. It was chosen for its efficiency in tip etching. The ideal tungsten rod has a diameter of $0.5 \mathrm{~mm}$. A wire this small is desired, since tips can be readily made with an apex diameter on the order of nanometers. It is not acceptable to have a wire with two to or more atoms on its extremity. This will result in the production of an image from multiple surface views, commonly called "multiple-tip imaging". Multi-image productions occur when electrons tunnel to the specimen from each of the apex atoms. Each view will account for an image which seems to have no characteristics whatsoever. A perfect scanning tip will have merely a single atom at the end. Having a single apex atom insures

that most of the tunneling current will pass directly from this closest atom. Thus, scanning will produce incredible atomic resolution.

\subsection{Electrochemical Procedure And Analysis}

The process used to make the tungsten rod sharp is called "electrochemical etching". Though the tungsten chemistry has been widely studied, the chemical kinetics are not easily found for this etching event. The reaction is certainly dependent on the specific type of current supplied to the electrodes. Etching procedures may be brought about by direct current (DC) or by an alternating current (AC). In this discussion an 
introduction to electrochemical kinetics is given for $\mathrm{AC}$ etching used in these experiments. For tungsten oxidation, the kinetics are given in FIGURE 3.

The etching apparatus is detailed in FIGURE 4. The tungsten wire serves as one of the electrodes. A circular cathode is easily made by painting the lower end of a hollow, cylindrical, plastic base with a conducting glue containing carbon. Dag 155 from the Acheson company has proven to work well. ${ }^{10}$ The probe electrode is centered within the circular electrode where the two are set in a beaker partially filled with two molar potassium hydroxide $(2 \mathrm{M} \mathrm{KOH})$. The aqueous solution can be produced by mixing 11 grams of solid $\mathrm{KOH}$ with 0.1 liters of water $\left(\mathrm{H}_{2} \mathrm{O}\right)$. Calculations for the solution mixture are easily obtained.

$$
\begin{aligned}
& 2 \mathrm{M} \mathrm{KOH}=\frac{2.0 \mathrm{moles} \mathrm{KOH}}{1.0 \mathrm{~L} \mathrm{H}_{2} \mathrm{O}} \\
& 2 \mathrm{M} \mathrm{KOH}=\frac{2.0 \mathrm{moles} \mathrm{KOH}}{1.0 \mathrm{~L} \mathrm{H}_{2} \mathrm{O}} \times \frac{56 \mathrm{~g} \mathrm{KOH}}{1.0 \mathrm{~mole} \mathrm{KOH}} \times \frac{0.10}{0.10} \\
& 2 \mathrm{M} \mathrm{KOH}=\frac{11 \mathrm{~g} \mathrm{KOH}}{0.10 \mathrm{~L} \mathrm{H}_{2} \mathrm{O}}
\end{aligned}
$$

The proper connections for the electrochemical process has the tungsten tip placed in series to an alternating current where $12 \mathrm{~V}$ are applied to the circuit (see FIGURE 4). A simple resister has been connected into the system for current readings. The $1 \Omega$ resistor continues is connected to a conducting screw in the top of the wire mount. The 


$$
\mathrm{KOH}(\mathrm{aq}) \Rightarrow \mathrm{K}^{+}+\mathrm{OH}^{-}
$$

$$
6 \mathrm{H}_{2} \mathrm{O}+6 \mathrm{e} \Rightarrow 3 \mathrm{H}_{2}(\mathrm{~g})+6 \mathrm{OH}^{-}
$$

$$
\begin{gathered}
\mathrm{W}(\mathrm{s})+2 \mathrm{OH} \Rightarrow \mathrm{WO}^{+}(\mathrm{s})+\mathrm{H}_{2} \mathrm{O}+3 \mathrm{e}^{-} \\
\mathrm{WO}^{+}(\mathrm{s})+2 \mathrm{OH} \Rightarrow \mathrm{WO}_{2}(\mathrm{~s})+\mathrm{H}_{2} \mathrm{O}+1 \mathrm{e}^{-} \\
\mathrm{WO}_{2}(\mathrm{~s})+\mathrm{OH} \Rightarrow \mathrm{WO}_{3} \mathrm{H}(\mathrm{s})+1 \mathrm{e}^{-} \\
\mathrm{WO}_{3} \mathrm{H}(\mathrm{s})+\mathrm{OH} \Rightarrow \mathrm{WO}_{3}(\mathrm{~s})+\mathrm{H}_{2} \mathrm{O}+1 \mathrm{e}^{-} \\
\mathrm{WO}_{3}(\mathrm{~s})+\mathrm{OH}^{-} \Rightarrow \mathrm{HWO}_{4}^{-}(\mathrm{aq}) \\
\mathrm{HWO}_{4}^{-}+\mathrm{OH}^{-} \Rightarrow \mathrm{WO}_{4}^{2-(a q)}+\mathrm{H}_{2} \mathrm{O} \\
\mathrm{W(s)}+8 \mathrm{OH} \Rightarrow \mathrm{WO}_{4}^{2-}(\mathrm{aq})+4 \mathrm{H}_{2} \mathrm{O}+6 \mathrm{e}^{-}
\end{gathered}
$$

\section{FIGURE 3. Electrochemical etching kinetics}




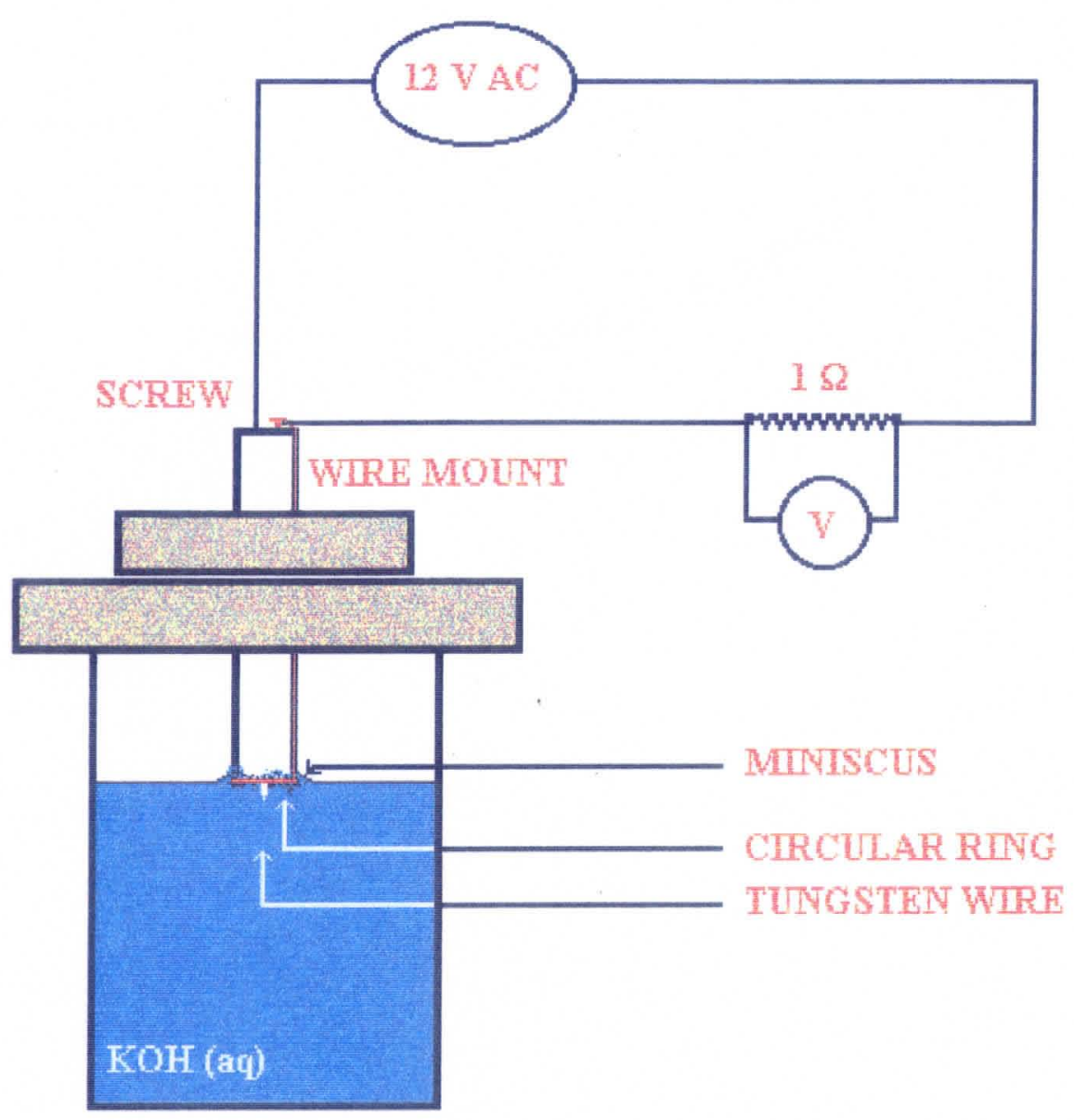

FIGURE 4. Oxidation-reduction apparatus connections 
hollow mount contains a hidden wire conecting the screw to the carbon electrode. The power supply completes the circuit between the screw and the resistor.

The wire mount should be lowered into the solution to the position where the painted base of the wire mount is just barely in the solution. Tension from the electrolytic surface causes the formation of a meniscus around the circular electrode and mount. The meniscus shape determines the ratio of the tip length to the wire diameter otherwise known as the "aspect ratio". ${ }^{11}$ It appears that the aspect ratio is the cause of the conical shape of the finished tip.

With an initial current reading of just less than $1 \mathrm{~A}$ across the resistor, the procedure is terminated when the current goes to $0.01 \mathrm{~A}$. At this time, the tip has reduced enough to rise above the solution surface within the meniscus region. The production of tungsten ions leaving the wire conically reduces the volume of wire remaining in the solution to a very fine point. With less wire to etch, fewer ions are released from anode to cathode. This accounts for the decrease in the alternating current passing through the cell. Between $20 \%$ and $30 \%$ of the volume may be taken from the end of the wire in typical electrochemical processes.

The duration of the etching event varies heavily on the length of the wire set in the solution. For a $0.25 \mathrm{~cm}$ typical tungsten wet-length, the elapsed time may be $185 \mathrm{~s}$. By timing multiple productions of tips with different wet-lengths and their initial current readings, it is possible to see the relationship between the wet-length and time as well as the wet-length and etching current. 
Wet-Length

(cm)

0.00

0.10

0.15

0.20

0.25

0.30

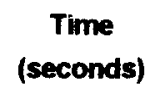

0.00

75.0

115

152

190

223
Current

(amperes)

0.000

0.244

0.375

0.499

0.620

0.740

Graphical analysis shows that there lies a hidden equation which approximates the relationship between the parameters for each of the two sets at constant voltage. The relationship of both sets of parameters is proportional. This is evident from GRAPHS 1 and 2. Denoting $l$ as the initial length of the tip in the electrolytic surface and $t$ for the time it takes for the etching process to take place, the relationship between $l$ and $t$ can be approximated.

$$
l=(0.001 \mathrm{~cm} / \mathrm{s}) t
$$

Similarly, by denoting $I$ as the current reading at the resistor, the relationship between $l$ and $i$ can be approximated.

$$
l=(0.4 \mathrm{~cm} / \mathrm{A}) I
$$


GRAPH 1. Wet-length versus time plot

\section{WET-LENGTH (CM)}

0.000

0.100

0.150

0.200

0.250

0.300
TIME (SECONDS)

0.000

75.00

115.0

152.0

190.0

223.0

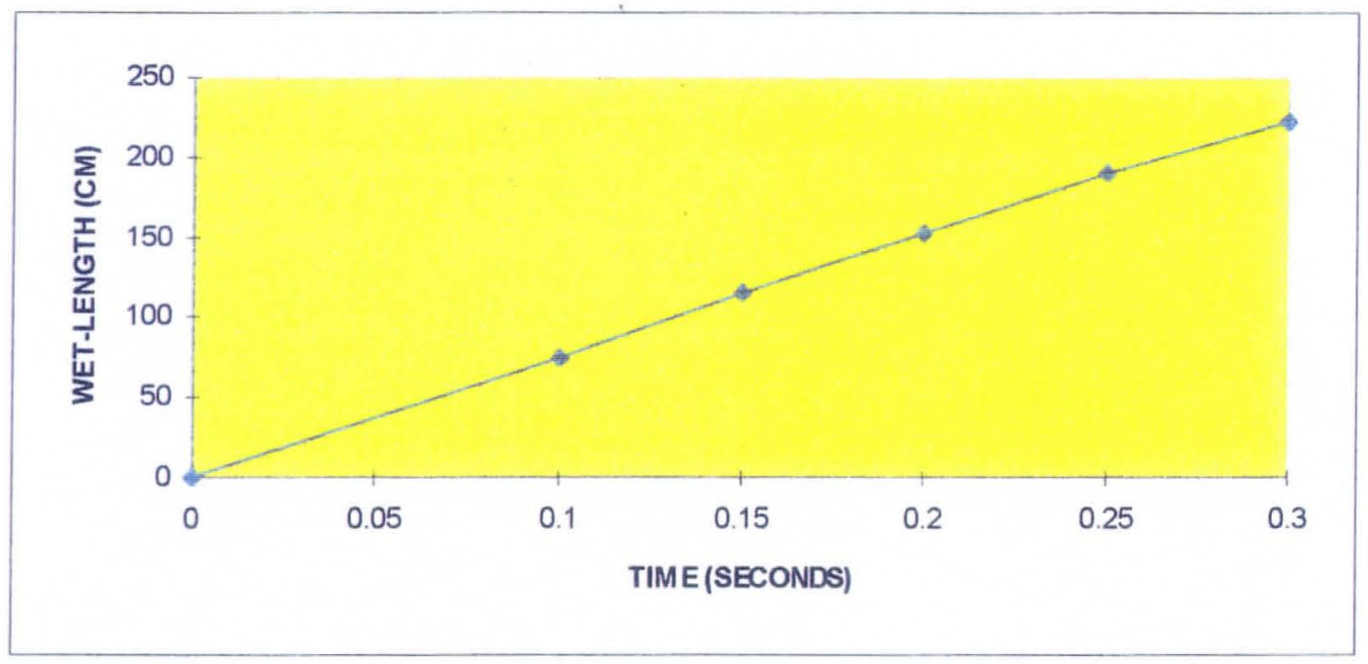

SLOPE $=0.00133 \pm 0.0000149$ 
GRAPH 2. Wet-length versus current plot

\section{WET-LENGTH (CM)}

0.000

0.100

0.150

0.200

0.250

0.300

\section{CURRENT (AMPERES)}

0.000

0.244

0.375

0.499

0.620

0.740

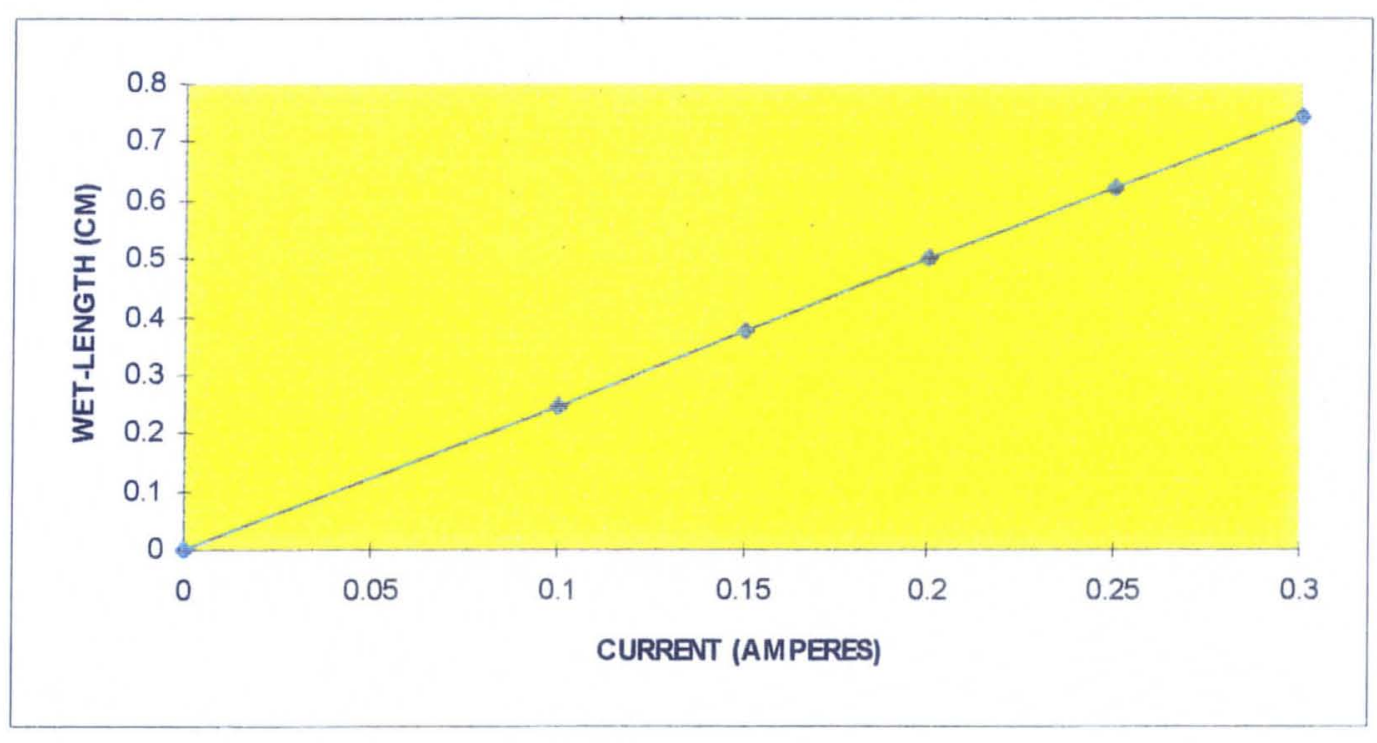

SLOPE $=0.404 \pm 0.00235$ 
Due to the fact that the parameters show a linear relationship, and must also be linear with respect to voltage, the aspect ratio and film tension can be said to cause the conical reduction of the polycrystalline tip. Researchers agree with these conclusions and add that another causal suspect could be the molar concentrations of the electrolyte. ${ }^{12}$

\subsection{Probe Finishing}

Following the production of the tip, its shape must be analyzed under a microscope. It has been proven that a magnification of $10 \times 2$ is sufficient. The shape should not be long and pointy, a characteristic of turning off the source too early. Nor should it be short and dull, a characteristic of turning off the source too late. An example of the perfect tip is shown in FIGURES 5 and 6. These pictures were taken from an Olympus SZH Zoom Stereo Microscope PM-10 AK with a C-35AD-4 Olympus Camera at $2 \times$ multiplication for the objective lenses. With a 6.3 light intensity, fiberlights were added for focusing. The first picture illustrates the miniaturization of the tip as it lies beside a $0.5 \mathrm{~mm}$ scaled ruler. The second picture shows a wire before the etching process and the finished product. The best approximation of the cone angle, with these pictures, is $27^{\circ}$

The atom at the tip of the wire must be a conducting atom. Nonconducting foreign deposits can cause $\mathbf{z}$-motion instability ${ }^{13}$ That is, the STM will sense a lesser tunneling current, causing the sample to move up towards the tip even farther. The effect might be inadvertent contact between the wire and the sample. These collisions are disastrous. 


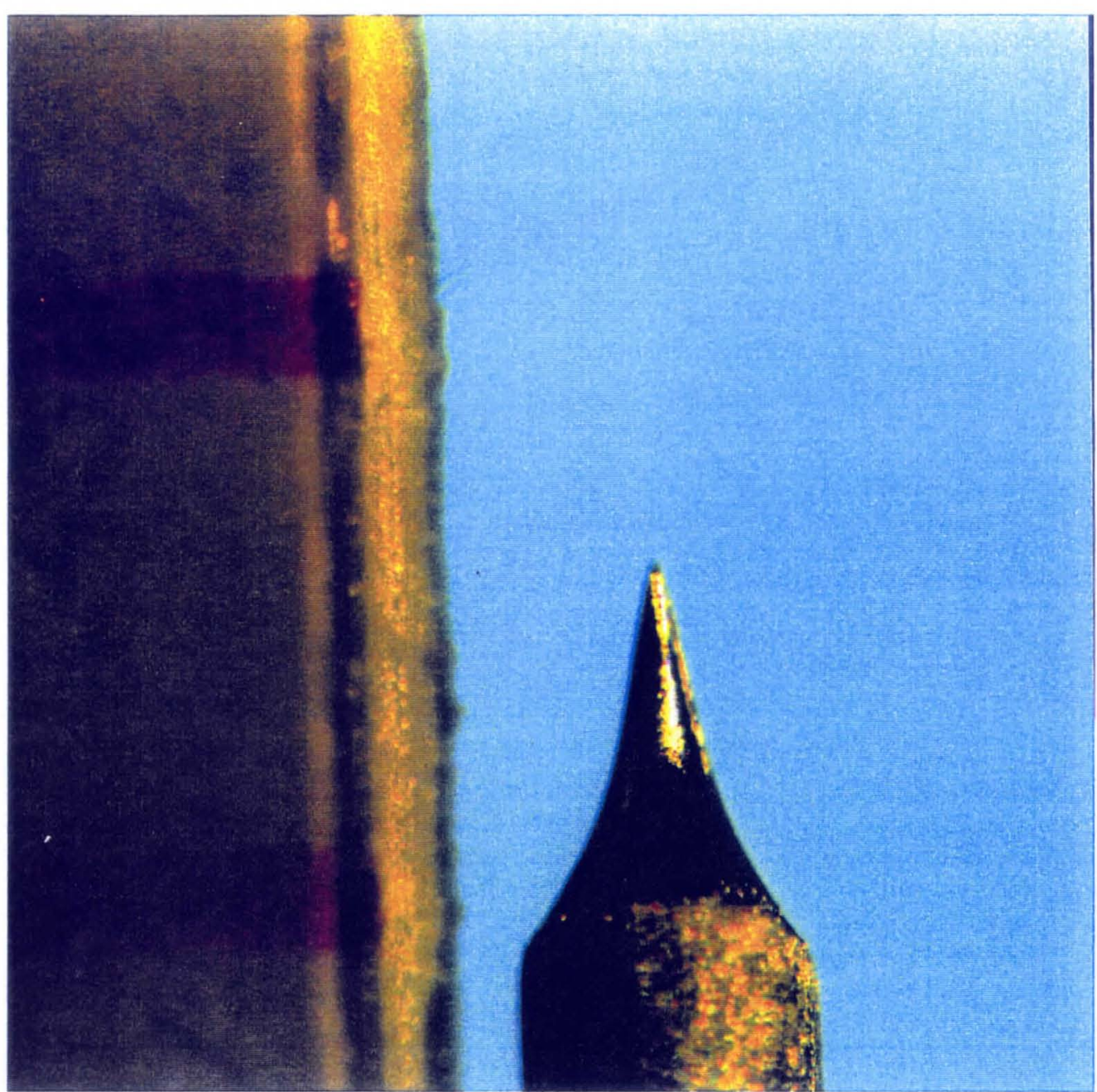

FIGURE 5. An etched tungsten tip beside a $0.5 \mathrm{~mm}$ scaled ruler 


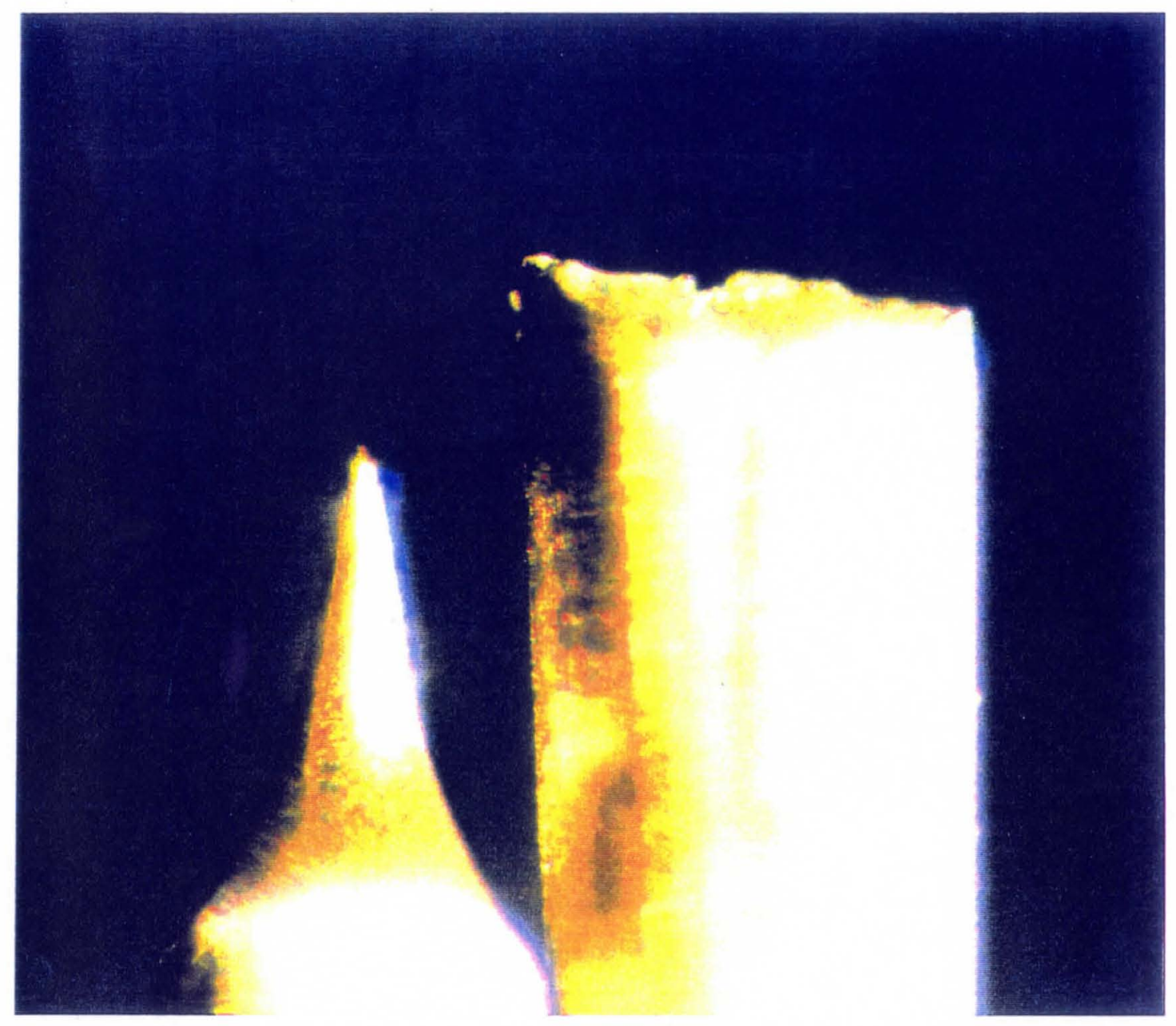

FIGURE 6. An etched tungsten tip beside an unetched wire 
Special care has to be taken before experimenting to insure that the tip is perfect and that the sample is very flat. When contact does occur, the procedure must start over.

Excess deposits such as potassium, tungsten dioxide $\left(\mathrm{WO}_{2}\right)$, and tungsten trioxide $\left(\mathrm{WO}_{3}\right)$ are commonly layered on tungsten wires as a result of the etching process. The build up occurs between layers at the end of the finished tip. In order to rid the chance of any instability with the STM, the processed tip is immediately set in hydrofluoric acid (HF) for 10 seconds, followed by distilled water, and set to dry. The hydrofluoric acid removes excess particulates that may have accumulated in the solution as well as unsymmetric layers of potassium, oxides, and tungsten fragments. Distilled water removes the hydrofluoric acid compounds similarly.

\section{$2.4 \quad$ Etching Kinetics}

Potassium hydroxide serves as the ionic compound which moves ions through the solution, constituting an electric current. The solution of $\mathrm{KOH}$ with $\mathrm{H}_{2} \mathrm{O}$ dissociates potassium hydroxide and water into potassium $\left(\mathrm{K}^{+}\right)$and an abundance of hydroxide $(\mathrm{OH})$ ions.

$$
\mathrm{KOH}(\mathrm{aq}) \Rightarrow \mathrm{K}^{+}+\mathrm{OH}^{-}
$$

Hydroxide ions are responsible for the beginning of the oxidation process at the tungsten tip. Hence, the power source can drive electrons from one electrode to the other when there exists a difference in energy of electrons at the two electrodes. With an 
alternating current, the polarity of the electrode changes with the sign of the potential. Hence, the polarity of the electrodes alternate with potential. At positive potential, the tungsten probe is positive and the carbon electrode is negative. The kinetics undertaken is understood by researchers to be that of an oxidation-reduction reaction. ${ }^{14}$

$$
\begin{gathered}
\mathrm{W}(\mathrm{s})+2 \mathrm{OH} \Rightarrow \mathrm{WO}^{+}(\mathrm{s})+\mathrm{H}_{2} \mathrm{O}+3 \mathrm{e}^{-} \\
\mathrm{WO}^{+}(\mathrm{s})+2 \mathrm{OH} \Rightarrow \mathrm{WO}_{2}(\mathrm{~s})+\mathrm{H}_{2} \mathrm{O}+1 \mathrm{e}^{-} \\
\mathrm{WO}_{2}(\mathrm{~s})+\mathrm{OH} \Rightarrow \mathrm{WO}_{3} \mathrm{H}(\mathrm{s})+1 \mathrm{e}^{-} \\
\mathrm{WO}_{3} \mathrm{H}(\mathrm{s})+\mathrm{OH} \Rightarrow \mathrm{WO}_{3}(\mathrm{~s})+\mathrm{H}_{2} \mathrm{O}+1 \mathrm{e}^{-} \\
\mathrm{WO}_{3}(\mathrm{~s})+\mathrm{OH} \Rightarrow \mathrm{HWO}_{4}^{-}(\mathrm{aq}) \\
\mathrm{HWO}_{4}^{-}+\mathrm{OH}^{-} \Rightarrow \mathrm{WO}_{4}^{2-}(\mathrm{aq})+\mathrm{H}_{2} \mathrm{O}
\end{gathered}
$$

The interesting phenomenon of this electrochemistry is that water is continuously being produced in the anodic oxidation of tungsten at positive potential. Water auto-ionizes to produce an abundance of hydroxide ions, aiding in the initial reaction of tungsten.

$$
6 \mathrm{H}_{2} \mathrm{O}+6 \mathrm{e}^{-} \Rightarrow 3 \mathrm{H}_{2}(\mathrm{~g})+6 \mathrm{OH}
$$


The excess of hydroxide changes the ph of the $\mathrm{KOH}$ solution. With more hydroxides in the solution, the concentration increases, thus increasing the ph level. ${ }^{15}$

$$
\mathrm{W}(\mathrm{s})+8 \mathrm{OH} \Rightarrow \mathrm{WO}^{2-}(\mathrm{aq})+4 \mathrm{H}_{2} \mathrm{O}+6 \mathrm{e}^{-}
$$

It is suspect that if the concentration of the solution is the cause of the conical form of the tip, it is the result of this rise ph. The rates of each of the chemical processes are different. The quicker of the steps occurs for tungsten trioxide molecule production. ${ }^{14}$

When the sign of the potential alternates to negative, then the polarity switches and the reaction is reversed. There is no $\mathrm{H}_{2}(\mathrm{~g})$ available for the reverse electrochemistry. Hydrogen gas, having a weight much less than water, bubbles out of the beaker when it is produced. Henceforthe, the positive driving potential is large enough to surmount the small negative potential. This leaves the reverse reaction negligable.

It is apparent that the electrochemistry would work better for DC etching, since one would not have to consider the negative potential. Also, for AC etching, half wave rectification would appear to do the same. Otherwise, the procedure is less controllable do to the spontaneous voltage drops from hydroxide concentration changes. Some rectification etching success has already been detailed for nickel tips in $\left(\mathrm{H}_{2} \mathrm{SO}_{4}\right)$ solutions. $^{16}$ 


\subsection{Production Alternatives}

An alternative to using tungsten as our probe would be to use a platinum-iridium (Pl-Ir) wire. The process of oxidation in an electrochemical process is not a factor for these wires. ${ }^{13}$ For the solution, an alternative to using potassium hydroxide would be the use of sodium hydroxide $(\mathrm{NaOH})$. Both are excellent electrolytes for an etching process.

Electrochemical etching is not the only method by which scanning probes can be developed. Ion milling is a very common practice as well. ${ }^{13}$ With this technique, probes are first treated with heat by electron beaming. The annealing process softens the scanning tip for shaping simplicity. The shaping procedure is undertaken by spinning the tip at a high velocity with an inert gas aimed at the shaping area. Gasses used in such a method are usually neon (Ne) or argon ( $\mathrm{Ar}$ ). If the gas stream is finite in diameter, it is possible to control the shaping of the wire.

Field Ion Microscopy is alsoan acceptional method for shaping tips. It is not as widely used as etching or ion milling since the apparati are more expensive to build. The method allows for atoms on a cut probe to be removed by an externally controlled electric field. ${ }^{17}$ By adjusting the tip potential, the electric field is characteristically adapted to expel single atomic rows from the apex. High speed neon atoms could then be projected onto the tip to reduce the final radius of curvature. ${ }^{17}$

These are but only a few of the tip fabrication possibilities today. There is no set experiment that works enormously better than the rest. The atomic resolution is nearly equal for all tip types. Many experimenters try to facilitate tip production with low cost 
standards. If this is the case, it seems logical to scan with the probe most simply made.

That is, one would find it pleasing to use electrochemical etching. 


\section{CHAPTER III}

\section{SCANNING TUNNELING MICROSCOPE SYSTEM}

\subsection{Microscope Apparatus}

The STM system consists of many different components. Each component has a unique importance for operating the system successfully. One of the most important of these is the microscope apparatus. The microscope apparatus is divided into two distinct parts. One part contains the tungsten tip and the other contains the sample. For easy identification, they are labeled in the discussion as the "tip apparatus" and the "sample apparatus".

Looking first at the tip apparatus, its operative pieces are micrometers and a piezoelectric tube. The finished tip is inserted into a probe holder on top of the piezoelectric tube. A small screw fastens the tip in the holder. It is important that the tip extend the surface of the holder by a length of $3 \mathrm{~mm}$ precisely. Tip vibration will result if the extension is much longer. If the length is shorter, the apex of the tip and the height of the probe holder with be so close that noise will occur from the tunneling of electrons on the conducting probe holder. Therefore, atomic resolution will decrease.

The exact explanation for the application of the piezoelectric tube will be given in a later section. In short, the tube bends in a direction that can be controlled by an applied voltage. A small bending change on a macroscopic level creates a large change on a microscopic level. With any $\mathbf{x}$ or $\mathbf{y}$ change in position, the tip will move over a different graphite location. Hence, the piezoelectric is used to move the tip to alternative locations on the graphite surface. The use of micrometers is similar. They are used to provide 
much larger movements than the piezoelectric can offer. Micrometers are connected to the base of the tube and are displaced prior to the experiment manually. A picture of the tip apparatus is shown in FIGURE 7. In the figure, all connections to other system apparati are indicated.

Secondly, looking at the sample apparatus, its operative piece is a stepper system. The stepper system functions to provide mechanical energy needed to raise the position of the sample and the mount close enough to be in range for tunneling to occur. FIGURE 8 shows the connections for this apparatus. The location of the stepper system is directly beneath the sample and the mount.

Once the sample and the tungsten probe are ready for experiment, o-rings are inserted in the median of each spacer. The two are then affixed together. Six screws tighten the aluminum spacers of each unit. This insures that there will be no flow of particles entering the hollow chamber to disrupt the tunneling phenomenon. The resulting connection is the full microscope apparatus.

The equipment is held in suspension by rubber cables in an aluminum cage. The cables disallow mechanical, contact vibration from anything in the environment of the system. The cage also provides enclosure to eliminate air flow disturbances. The electrical connections made from the comparator, pre-amp, and model 100 STM controls are all done through insulated holes in the cage. Like rubber cables, the insulation stops mechanical vibration in the wires. The entirety of the Scanning Tunneling Microscope system is given in FIGURE 9. 




FIGURE 7. Scanning tunneling tip apparatus 


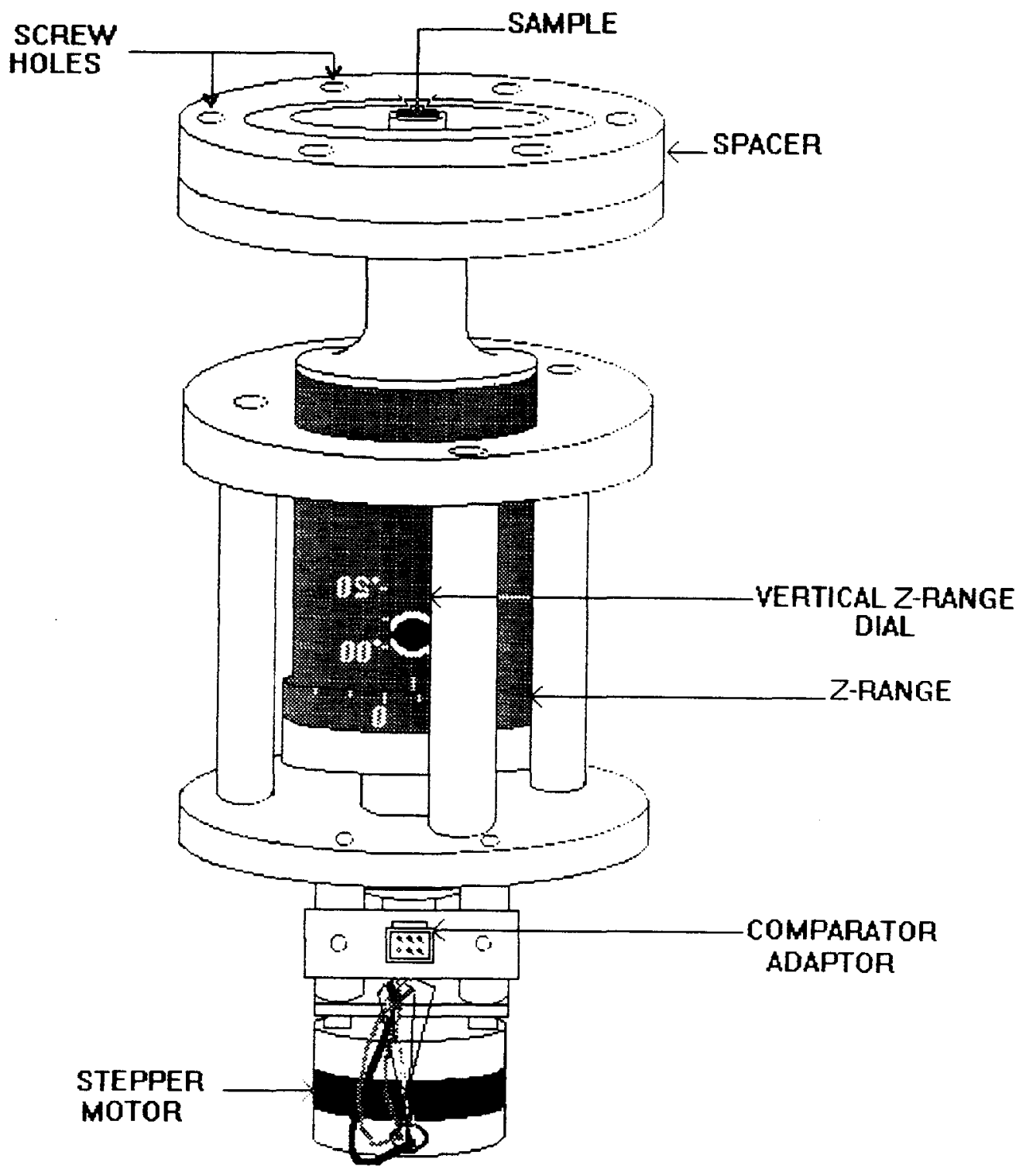

FIGURE 8. Scanning tunneling sample apparatus 


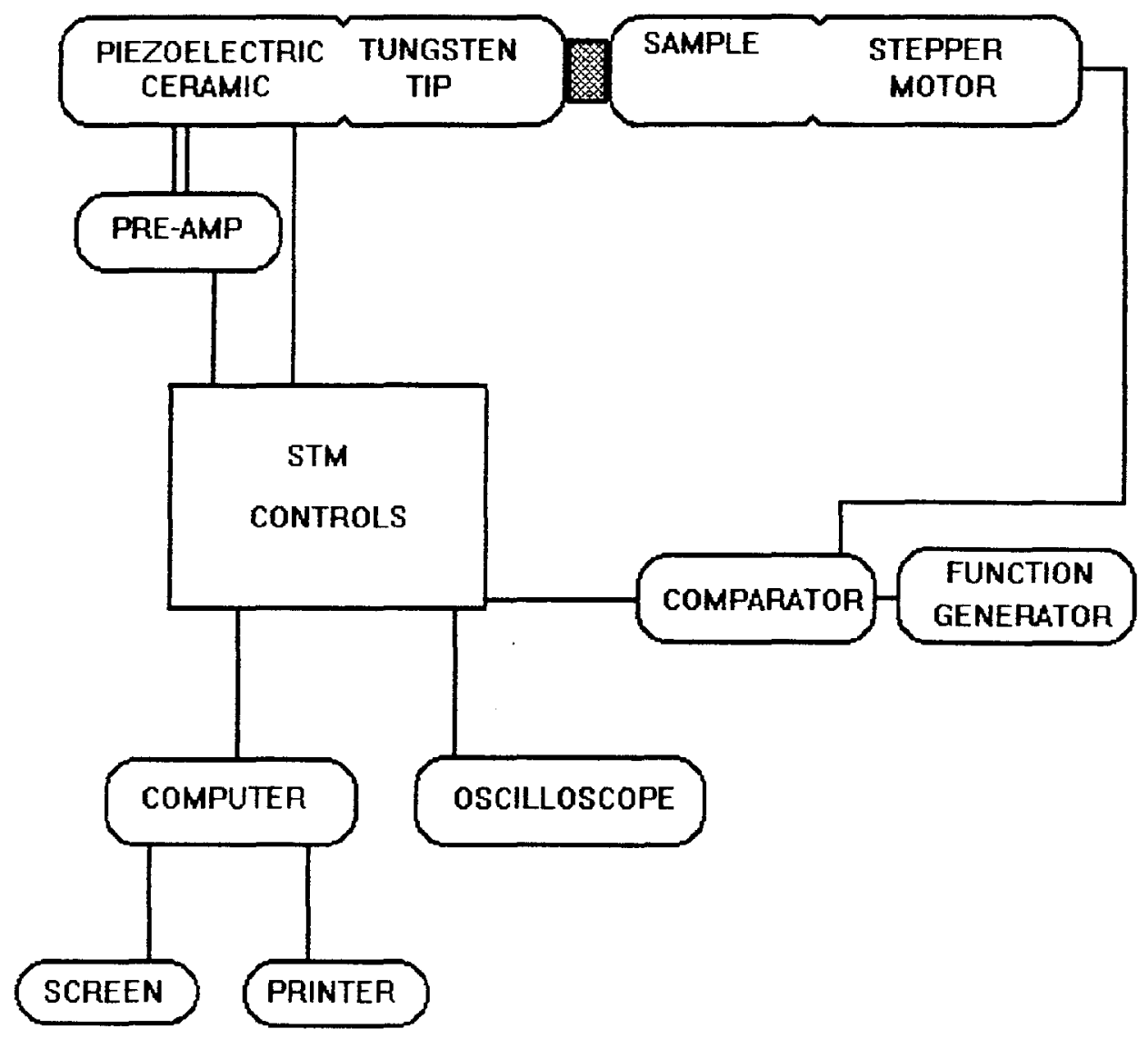

FIGURE 9. Scanning tunneling microscope system 


\subsection{Sample Lift Mechanism}

In order to bring the sample close enough to the tip for tunneling to occur, there must be a mechanism for movement beneath the sample. The mechanism used to provide this movement is called the "stepper motor". Factory specifications require that the motor move in steps at a rate of 200 steps per revolution. It had been found that the cycle time for approach could be lessened if the motor moved through more steps in each cycle. In order to achieve better control and reduce the time it takes to bring the sample into tunneling range a reduction gear could be introduced along with the stepper motor. Specifications for the mechanism included in this system indicate that its application increases unidirectional movement by a gear ratio of $75: 1$.

The angular velocity of the stepper motor with the reduction gear alone will do nothing to raise the sample without another mechanism used to generate vertical displacement. A linear motion vacuum feedthrough unit was then connected for variable position change. One inch of disposition is provided per 200 revolutions. ${ }^{18}$ As the stepper motor provides the forced motion, the three units combine to raise the sample to nearly 85 $\AA$ per step. This calculation is done below.

$$
\begin{gathered}
\Delta \mathrm{z}=\frac{1 \text { revolution }}{200 \text { steps }} \times \frac{1}{75} \times \frac{1 \text { inch }}{200 \text { revolutions }} \times \frac{0.0254 \mathrm{~m}}{1 \text { inch }} \times \frac{10^{10}}{1 \mathrm{~m}} \frac{\AA}{-} \\
\Delta \mathrm{z} \approx 85 \AA / \text { step }
\end{gathered}
$$


This rate of change is the same regardless of whether or not the graphite is being raised or lowered in the sample apparatus. There is a dial on the external body of the STM control system which will center when tunneling range is achieved. Once the dial moves to the center position, the signal will be given to stop the approach procedure. When the sample reaches nearly $70 \AA$ from the tip, the approach current is automatically shut off by an RHK Technology comparator and a Forthright Electronics stepper driver card. ${ }^{19,20}$

\subsection{Comparator And Driver Card}

The comparator and stepper driver card are communicating circuits designed to operate the stepper motor. The two circuits are not part of the sample apparatus. They are external circuits affiliated with the STM control unit to either activate or enable the sample from being mechanically driven.

The comparator initializes the commands for stepper motor mobility depending on the level of input voltage. If the comparator deduces that the level of the input voltage is less than a reference voltage, it sends a signal to the driver card. The driver card activates the stepper motor at that time. The motor can be authorized to approach the sample to the tip or to retract from it. The motor will run in reverse if the current passes through it in the opposing direction of the approach path. A switch provides this choice of motion.

One would see a display of $-130 \mathrm{~V}$ on the digital voltmeter when the sample is out of range. The digital voltmeter is positioned on the face of the STM control unit. This reading indicates that the voltage being applied to the comparator is $-1.30 \mathrm{~V}$. The reference voltage for all experiments has been previously set in the circuit to $-1.36 \mathrm{~V}$. 
With the applied voltage being the lesser of the two, the comparator signals the driver card to allow motion.

If the switch was engaged for approach, tunneling current in the circuit would commence when the sample is roughly $100 \AA$ from the probe. Motion by the stepper motor will continue until the comparator surpasses the reference voltage. At an applied voltage of $-1.37 \mathrm{~V}$, surpassing the reference voltage, the comparator will order the driver card to inhibit motion beyond its present location. The sample will drift toward the tip for a short time. The final digital voltmeter reading will conclude in the range of $-1.4 \mathrm{~V}$ to $-0.70 \mathrm{~V}$ nominally, as the stepper motor comes to a halt. Further motion would require a single steps.

A single step option is made possible by a button connected to the comparator circuit. The option usually warranted for retraction only. The tip may crash with a single step of nearly $58 \AA$. Stepping may induce more drift as well. In order to disallow further drift and mechanical noise to occur, the comparator should be turned off once the tunneling range is acceptable.

\subsection{Function Generator}

The function generator controls the speed at which the shaft in the stepper motor cycles. A large frequency provides more cycles per second than does a smaller frequency. Experiments have shown the stepper motor to drift to nearly $30 \AA$ after the order has been given for the comparator to cease motion. This concludes that the threshold for 
laboratory frequency must be withheld to prevent crashing. Past experiments have justified a reasonable operating frequency to be below 120 cycles / $\mathrm{s}$.

\subsection{Pre-Amplifier}

Interference makes its presents well known in the Scanning Tunneling experiments. The main source of background noise lies within the tunneling signal. Before the STM control unit relays the tunneling current to the computer, it is first intercepted by the preamplifier.

The occupation of the pre-amplifier is to differentiate between the electrostatic wire interference and the true tunneling current signal. As the current is differentiated, the tunneling signal is amplified. The amplification limits the order to which noise is efficient. The tunneling current may then flow to the STM controls for relay.

\subsection{Oscilloscope}

The tunneling current signal carries the impulses from scanning across the atomic surface. Each atom close enough to permit tunneling will conduct a single current if the tip has but one apex. As the tip moves back and forth, the individual currents are superposed to constitute a total current. This superposition is visible on an oscilloscope.

Oscilloscopes measure electrical impulses and present the signal in the form of a graph on a cathode ray tube. Since impulses can be timed, and are associated to some frequency of voltage, a graph of voltage versus time is possible. 
Ripples on the grid of the oscilloscope portray the atomic tunneling currents. The similarity in the size of the ripples illustrate that the test surface contains the same structure and that the structural area is smooth. The application of the scope is not for the sole purpose of looking at the equivalency of the impulse amplitudes from graphite atoms. The primary application is for noise analysis. It is unfortunate that the pre-amp will not rid all of the interference present in this experiment. Some smaller, unwanted, electrostatic impulses have a presence which distorts the trace pattern. If the noise continuously disturbs the impulse shape, then it may have been induced by more than just electrostatics. It has been seen that tungsten probes exhibit these effects when picking up compounds like tungsten dioxide and tungsten trioxide on the apex. Another possibility is that the tip length is unsatisfactory. Tip vibration from the scanning oscillation may be the cause of trace noise.

\subsection{Piezoelectric Tube}

The ceramic material used for tip displacement is made of lead-zirconate-titanate $\left[\mathrm{Pb}(\mathrm{Zr}, \mathrm{Ti}) \mathrm{O}_{3}\right]$, otherwise known as "PZT". Piezoelectricity is a physical phenomenon that has been described in detail in many articles. It is best stated as "an interaction between electrical and mechanical processes". ${ }^{21}$ As mentioned earlier, the purpose of the ceramic material is to provide vertical and lateral movement for the scanning probe. The piezoelectric material is used for its incredible ability to bend at atomic scale distances with respect to a voltage applied to its surface. 
There are different types of scanners used in tunneling experiments. Some of the designs available are bar tripods, single tubes, tube tripods, and stacked disks. The scanner used in this system is one of single tube design. In a single tube set-up, the piezoelectric has the shape of a cylinder. The tube rests directly beneath the probe holder in the tip apparatus. It is the case for design that the tube be hollow enough in the center to hide the tunneling current wire extended from the base of the probe holder. The core was fabricated to a thickness of merely $2 \mathrm{~mm}$. Its total length is only $16 \mathrm{~mm}$. Due to its sub-micrometer usage, the tube is indeed miniature in size. A diagram for the leadzirconate-titanate ceramic can be seen in FIGURE 10.

In single tube designs, four separate quadrants of equal shape and size are affixed to the sides of the cylinder. On each quadrant lies a conductive band. When a voltage is applied to a particular band, the scanner bends perpendicular to the axis that quadrant was affiliated with. The deflection direction will be in the opposite of the sign of the electrode's potential. As current passes through the electrode, the ceramic will bend in the direction orthogonal to their location. It is possible to move the scanner bidirectionally in $\mathbf{x}$ and $\mathbf{y}$. That is, the voltages may be chosen unsymmetric to bend the piezo off center. ${ }^{22}$ For example, the piezo will bend at an angle of $45^{\circ}$ if the $\mathbf{x}$ bands receive twice as much potential as the $y$ bands. One must make a note that the ceramic will extent forward at least to some extent when applying any increasing potential change.

For vertical motion, all four quadrants must be given the same voltage, such that no orthogonal bending is influenced. The equal concentration will cause longitudinal bending along the length direction of the piezo tube. There is another piezo attached to 


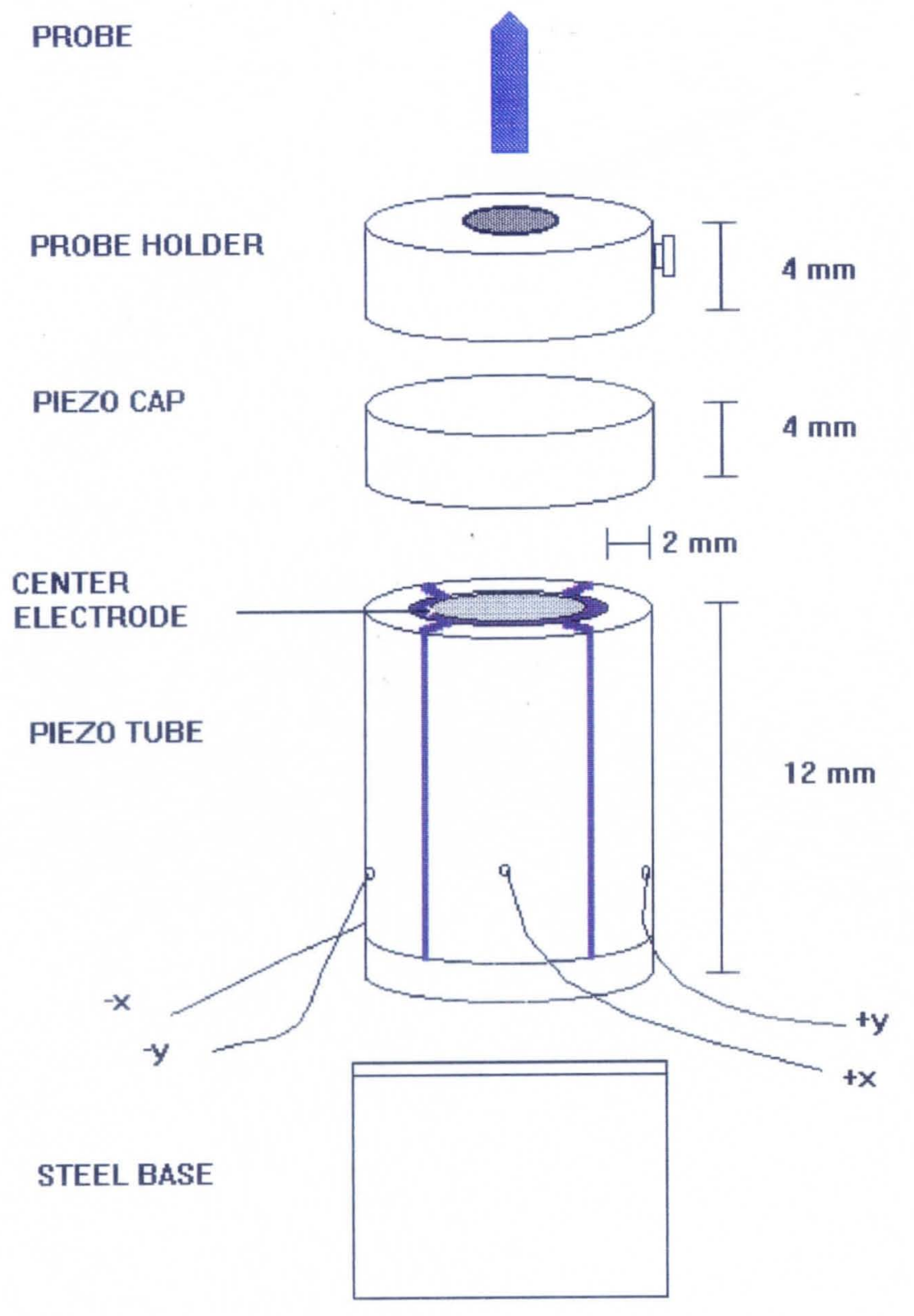

FIGURE 10. Piezoelectric lead-zirconate-titanate ceramic 
the bottom of the tube. This serves to uniformly expand the piezo in the $\mathbf{z}$ direction as well. The connection for this $\mathbf{z}$ piezo is at ground for all of the following experiments. Therefore, in these experiments it is only possible to move the tip in the $\mathbf{z}$ direction by applying an equal magnitude of voltage to all four bands on the outside of the tube. The lateral displacement has been found to approximately obey an equation where the motion is dependent directly to the applied voltage. ${ }^{13}$

$$
\Delta \mathrm{l} \approx \mathrm{d} 31 \frac{\mathrm{V} 1}{\mathrm{t}}
$$

The constant of material $d 31$ is the ratio of the strain in the length $l$ axis to the applied electric field in the thickness $t$ axis. ${ }^{23}$ An approximate value of this constant, corresponding to the size of the piezo tube is around $45 \AA$ / V.20 Substituting in this value, the value of the length and width of the PZT sections stated in FIGURE 10, and a typical moderate potential of $0.1 \mathrm{~V}$, one obtains a result for the $\mathrm{z}$ movement of the PZT ceramic.

$$
\Delta \mathrm{l} \approx\left(45 \frac{\AA}{\mathrm{V}}\right)\left(\frac{12 \mathrm{~mm}}{2 \mathrm{~mm}}\right)(0.1 \mathrm{~V}) \approx 27 \AA
$$

This is a very rough estimate for piezo bending. The exactness of the equation is irrelevant, since creep and hysteresis are present when one uses piezoelectrics in a Scanning Tunneling Microscope. ${ }^{13}$ 
The problem with creep is the nonlinear response time of the piezo. It is a frequent occurrence that the piezo will not respond immediately after the potential is set to one or more metallic bands. It may be seconds before potentiometers detail tip movement. In general, a large percentage of the movement is done instantaneously, with the remaining displacement lagging in time. There are other situations that occur where the piezo undergoes movement, even in the absence of potential, that can be considered the main characteristic of a system undergoing creep. The situation is very similar. It is simply a slow motion that the piezo retains after a large potential was given. ${ }^{13}$

Hysteresis in PZT is not uncommon to tunneling experiments. It is a problem that many such ferroelectrics have where there is no linear response to an applied electric field. The problem is explained considering an electric field $\boldsymbol{E}$ and a vector field $\boldsymbol{D}$ increasing to nearly the saturation point of a molecule's dipole alignment. If the fields are slowly decreased from saturation, the path of $\boldsymbol{E}$ versus $\boldsymbol{D}$ will not be retraced. Thus, in PZT, there is no direct proportionality between the two which resembles $D=\varepsilon_{0} \boldsymbol{E}+\boldsymbol{P}$ where $\boldsymbol{P}$ is the polarization vector. ${ }^{24}$ It is believed that the ceramic may be in a state of irregular polarization. ${ }^{24}$

Though all of these problems will be to some degree active in this system, they can be reduced by what is termed the "capacitor insertion method". ${ }^{25}$ Research has proven that by inserting a capacitor into series with the four quadrants of the ceramic, that by dividing the voltage between the capacitor and the scanner, excess fluctuations can be reduced. Maylor capacitors of efficiency $47 \mathrm{nF}$ on the $\mathbf{x}$ band and $15 \mathrm{nF}$ on the $\mathbf{z}$ band can provide a less sensitive system. ${ }^{25}$ 


\subsection{Model 100 STM}

The Scanning Tunneling Microscope control unit is an RHK Technology System model $100 .{ }^{19}$ The system is designed to give maximum control over probe scanning velocity, piezo movements, and resolution if the settings are appropriately dialed. The electrical control and adaptability of the RHK unit is excellent. The most impressive image resolution is achieved when the tip moves the slowest during the scan. It is for this reason that the raster scan generator is controlled by the model 100 STM. Scan rates can be increased if the sample is flat and the image is of high quality. Different scanning rates are changed with the millisecond per line switch. The rate may be increased by either increasing the number of seconds it takes the tungsten wire to probe each line, or by increasing the value of the multiplier dial. The multiplier may lift the scanning time by a factor of 1000 . The slowest scanning rate corresponds to a setting of 1000 multiplication of a $9 \mathrm{~ms}$ / line scanning time. Thus, at 9 seconds per scan, the best acquired image is possible.

Another valuable component in the model 100 STM is the analog edge meter. This is a scale which shows the relative $\mathrm{z}$ position with respect to the feedback loop voltage. When the scale is centered, the tip is in its dynamic range for tunneling. When the range is not sufficient, the $\mathbf{z}$ offset will permit recentering.

As the tip is stopped in range for tunneling, the analog edge meter should be set for A / D output. This will occur when the A / D gain is greater than $1 \mathrm{~V}$. The higher the 
gain from the converter, the more substantially noise is reduced. The converter voltage can be optimized by the $\mathbf{z}$ offset changes also.

The operation setting for these experiments is constant current mode. In this mode, small changes in the atomic surface will not disturb the feedback loop tunneling current response as much as if the current could vary. With the model 100 STM set in linear feedback mode at a specific current setting, acquisition can be attained when the current exceeds that level. The current mode for tunneling experiments is set initially at $\operatorname{lnA}$

The STM also has scan and offset functions for the single tube scanning head used. The piezoelectric material was stated as having a "four quadrant design". Two of the quadrants were for the $\mathbf{x}$ elements and two of the quadrants were for the $\mathbf{y}$ elements. Each of the two sets of directional quadrants are capable of independently changing the scanning range of the tip when the offset is changed. The change can be made from separate dials on the model 100 STM. There is a -130 to +130 variable voltage available to go to each of the elements in the ceramic. The voltage must be divided between the two settings. As a precaution to eliminate noise, the absolute value of the range and the offset should add to no greater a value than $130 \mathrm{~V}$. It has been seen that the amplifiers exhibit noise when saturated.

Range and offset dials are related to the bending of the piezoelectric ceramic. Since the underlying ceramic is not connected to give $\mathrm{z}$ offsets alone, the range and the fine offsets in the $\mathbf{z}$ direction can be evaluated by summing the voltages of the $\mathbf{x}$ and $\mathbf{y}$ elements equally. 
As a result of these offsets, the topographic images are plots of the feedback loop output for $\mathbf{z}$ displacement as a function of the $\mathbf{x}$ and $\mathbf{y}$ position above the sample. Since the atomic scale range has the characteristic of changing in real time, the constant current mode allows for only an instantaneous $\mathbf{z}$ current graph.

When the tip is stationary in its dynamic range and the image is obtained, it is often the case that darkness appears on the pictures extremity. The darker side of the image is a result of sample surface tilt. When the tilt is visible on the topographic image, one must use the compensation dial to correct for the shady projection of tilt. The model 100 STM subtracts the slope of the topograph from various cross sections. This dial is usually disengaged to allow better compensation from the software implemented.

\subsection{Display Software}

The model 100 STM communicates with a computer to decipher the current and voltage readings before it is sent for display. The computer and screen used are Zenith Data Systems models. Their class numbers are Z386 / 25-M150and ZCM-1490-Z respectfully. The program used to sort and graph the current data conveyed from the computer is STimage Version 2.0. ${ }^{19}$ The program is another product of RHK Technology, Incorporated.

STimage has the capability to detail current readings into graphics at any time during scanning. Its design enables visualization of cross sectional data, as well as two and three dimensional pictures. For two and three dimensional representations, the polar coordinate angles to which the pictures are given, may need a different view. 
Conveniently, the program is a tool that can be used to provide options of rotation and tilt The view can be taken from either of two pictures acquired during the probe's for and aft scanning motion.

The numerical topographic data exhibited in the program is distinguished for its real time computations. STimage provides information regarding scanning area, gap resistance, sample bias, and various other values. Since Microscopy relies heavily on the pictures produced to support Atomic and Surface Physics claims, it is very important to include in any system, a program which gives the best possible representation of the sample surface. STimage provides this necessity. 


\section{CHAPTER IV}

\section{CALIBRATION METHODOLOGY : EXPERIMENT I}

\subsection{Sample Preparation}

The sample used in the following experiments is Highly-Oriented Pyrolitic Graphite (HOPG) obtained from the Advanced Ceramics Division of Union Carbide. The volume of the sample is $4 \mathrm{~mm} \times 4 \mathrm{~mm} \times 4 \mathrm{~mm}$. It is very important that samples used with a STM be free from dust and all other contaminating molecules. If there exists any object above the sample surface which is non-conductive when scanning begins, there will be no flow of current to that particular object. In essence, the tip will recognize the sample as being farther than it really is. A couple of problems may occur if this is the case. First, as the tip approaches the sample, the probe may make contact with the object. The experimenter would then have to retract the tip and electrochemically etch a new one as well as prepare a new sample. Secondly, if contact is not made, the topographic images may be filled with so much random noise that it would be impossible to make sense of the real surface features.

In order to remedy the possibility of sample contamination, one should remove the top few layers of the sample prior to each experiment. This process is termed as "sample cleaving". Cleaving can be easily achieved for ZYA grade graphite samples. Pressing a strand of regular clear tape onto the face of the sample will bond the top layers to the adhesive molecules on the tape. If the tape is removed slowly, the adhesive molecules will take with it the top few layers of the graphite sample. It is the top layers which contain the contaminating elements, compounds, or dust which cause problems for tunneling 
experiments. With these objects no longer present on the sample, experimentation of structural phenomena will result in a pure study of the carbon surface. One may wish to use a lens of magnification $1 \times 10$ or higher to determine whether the scanning area is flat enough for study. Layers of carbon may be loose on the surface after the cleaving process. This could cause crash events as well. The experimenter must recleave the sample until the surface is clean and extremely flat. Once this is done, the carbon sample may be placed in the sample apparatus. If the tip has been etched properly, it may too be inserted into its tip apparatus. An o-ring is placed in the slot on top of the sample apparatus, allowing the two units to be connected. These two apparati together make up the entire STM system. Care should be taken to assure that the bolts of the system are clasped firmly. All electrical connections made to the model 100 STM, pre-amplifier, and comparator should not be loose, and there should be nothing touching the STM as it hangs by the rubber cables in the aluminum cage. It is advisable to allow 20 to 30 minutes to pass before initializing the comparator. The delay in time is sufficient to slow the simple harmonic motion of the system to a halt. At this time the control panels can be turned on and experimentation can begin.

\subsection{Calibration Hypothesis}

The following experiment is an analysis devoted to correctly calibrating topographic images. The first study will be that of calibrating measurements on the surface or the c-plane of the sample. A technique for calibrating such longitudinal measurements may be achievable by comparing the amplitude spacing of a cross section 
plot for a row of atoms versus the known atomic spacing of atoms. A flat surface area of graphite will be used for this observation.

A second analysis will be undertaken to calibrate measurements regarding vertical $\mathbf{z}$ variation of the sample. This lateral technique may be achievable by comparing height changes on the surface to the known atomic spacing of layers. A surface area containing various steps will be utilized for these $\mathbf{z}$ range observations.

\subsection{Calibration Review}

All molecules which are in solid phase have a definite structure. Whether or not the molecules are carbon type graphite, diamond, or a fullerine, they will bond in specific patterns. Graphite is the most widely used sample in STM experiments. It has been a source for new artifacts for many years. Its good conductivity, easy maintenance, and adventurous dislocation and step boundaries have attracted a vast majority of researchers. It is the typical step boundary structures that will be studied in the following experiments.

Graphite is well known for its honeycomb shaped structure. This has been proven elsewhere by $x$-ray diffraction techniques. Six graphite atoms are connected together in the same plane. When atoms have this structure the array is termed "honeycomb". It is interesting that the honeycomb structure is not at all easy to discover with an STM. Instead, observers see is a closed packed hexagonal structure.

Ouseph, Poothackanal, and Mathew have published on this very subject. ${ }^{26}$ Three of the six atoms on the honeycomb array are called "B-atoms" while the other three are termed "A-atoms". ${ }^{26}$ There are never two B-atoms or two A-atoms directly connected to each other in the same layer. The elements are paired according to the rule A-B-A-B-A-B 
around the honeycomb. ${ }^{26}$ The B-atoms can be identified in each array of FIGURE 11 by the red colored circles. The blue shaded circles represent the A-atoms. Placements are easy to identify when STM topographs appear as honeycomb structures. There is exactly one new honeycomb adjacent to each A-atom. ${ }^{26}$

Careful observation of the three dimensional diagram would allow one to see how each layer is connected to other layers. Directly beneath A-atoms in the first layer are Batoms. The connection of those B-atoms are to A-atoms in the third layer. The pattern is symmetric throughout the depth of the sample. However, B-atoms in the first layer are directly above the center of honeycombs in the second layer. Thus, there is no atomic connection for the B-atoms in the lower layer. In the third level the B-atom is connected to other B-atoms.

In consequence of A-atoms and B-atoms having different structures, the energy levels of A-atoms are different from B-atoms. That is to say their conductivities differ. The unsymmetric electronic energy distribution for the two types of atoms leaves a closed packed "B" structure for most tunneling phenomena, since it has a radically higher conduction in comparison. ${ }^{26}$ This explains why the honeycomb is usually not seen. Other reasons for not seeing the honeycomb shape in topographs may be because of tilting and $\mathbf{x}$-axis and $\mathbf{y}$-axis scaling differences.

Research was presented as to why the honeycomb is sometimes visible. If the top layers are misaligned, the stacking arrangement is altered. ${ }^{26} \mathrm{~A}$ few of the A-atoms may not have sublevel atoms nearby, and therefore will not all have different energies as was the case with the B-atoms. There will be a shift in tunneling participation where some of 


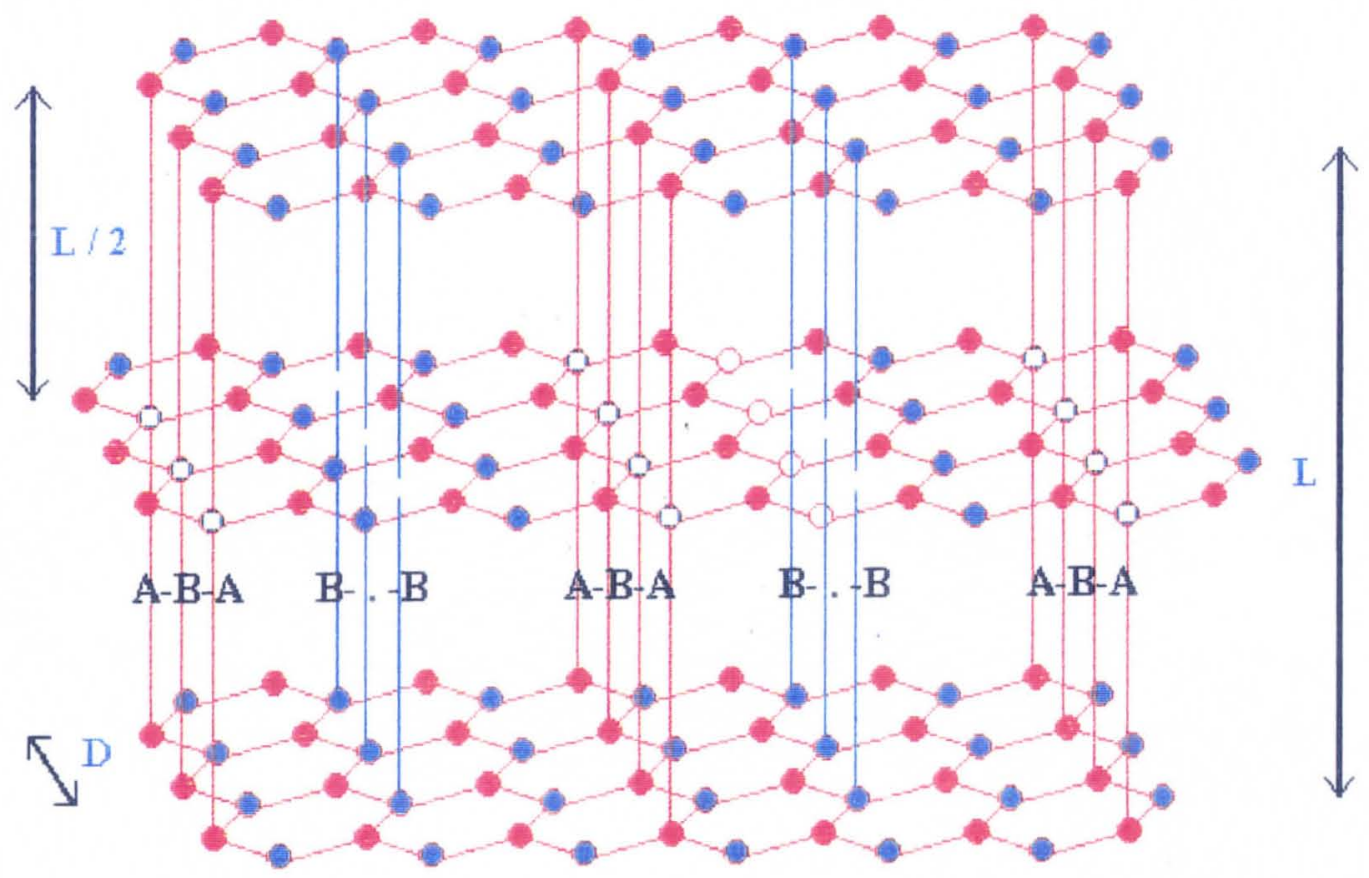

FIGURE 11. 3D carbon-graphite structure diagram 
the B-atoms will no longer conduct electrons, some of the A-atoms will. This misalignment results in honeycomb shaped topographies. ${ }^{26}$

In these experiments, the closed packed hexagonal symmetry-will be viewed. It is important to understand the fundamentals of the carbon-graphite structure when one is seeking to calibrate images based on the known structure. The concept is to utilize the lattice spacing constant $D$ and the layer spacing constant $L$ for graphite to determine the correct scaling for these measurements in STM images. As was illustrated in the previous figure, $D=2.46 \AA$ between each $\mathrm{BB}$ and $\mathrm{AA}$ pair and $L / 2=3.345 \AA$ between each layer. $^{8,26}$

\subsection{Calibration Experiment}

During experimentation current is kept in a constant mode at approximately $1 \mathrm{nA}$. The offset dials are continuously changed throughout the scanning process until topographs show atomic resolution. FIGURE 12 is a two dimensional topograph of the graphite sample in excellent atomic resolution. On this flat surface area the small, symmetric peaks are representative of each atoms' position. If one were to look at the variation of height of the B-atoms along any row, one would see what is termed as a "cross section". A-atoms and vacuum spaces conduct none of the electrons emanating from the tip, so nothing is imaged at those localities.

STimage software allows one to draw a cursor line across a row of atoms on the topograph. When this is done the program displays a lateral profile for amplitudes. The cross section in the figure is scaled in length to $80 \AA$. Within the graph, there are 




FIGURE 12. Uncalibrated cross section in the $x-y$ plane 
approximately $17 \pm 0.05$ peaks. Each represents one carbon atom. If the cursor on the topograph was long enough to fill the entire cross section with peaks, there would be 20.7 \pm 0.05 peaks - one for each atom. We know that the distance of separation between atoms along a row of the same layer of graphite is exactly $2.46 \AA^{26}$ Therefore, with 20.7 \pm 0.05 atoms in lateral view at a $2.46 \AA$ spacing, the range limit should be $51 \pm 0.05 \AA$. STimage provided an incorrect reading in the $\mathbf{x}$ and $\mathbf{y}$ direction. The true scaling should be a factor $F_{x, y}=0.64 \pm 0.03$ less. With a $51 \pm 0.05 \AA$ limit, FIGURE 13 would be the correct calibration image for the lateral measurements. Experiments for the future could be calibrated in the same manner. This should be done at all times when a resolution of the atomic sites is apparent.

Calibrating the vertical $\mathbf{z}$ measurements is a little different, because one must evaluate the varying heights of the structures. Steps of graphite will be used here. The product of scaling will be accomplished in a manner which is similar to that seen in the classic oil drop experiment. In 1909, Physicist R. A. Millikan used water vapor and oil droplets to determine the charge and mass of the electron independently. His result for the total charge of an oil drop was integer multiples of the charge of the electron. Here, I wish to determine the integer number of layers existing between elevated step structures on graphite.

Millikan used a stopwatch to record the time it took for charged water vapor to move in response to an electric field existing between two plates. He realized that he could determine the total charge of the vapor by accounting for all of the forces in the system, leaving only the terminal velocity of the vapor as the unknown variable. With an 


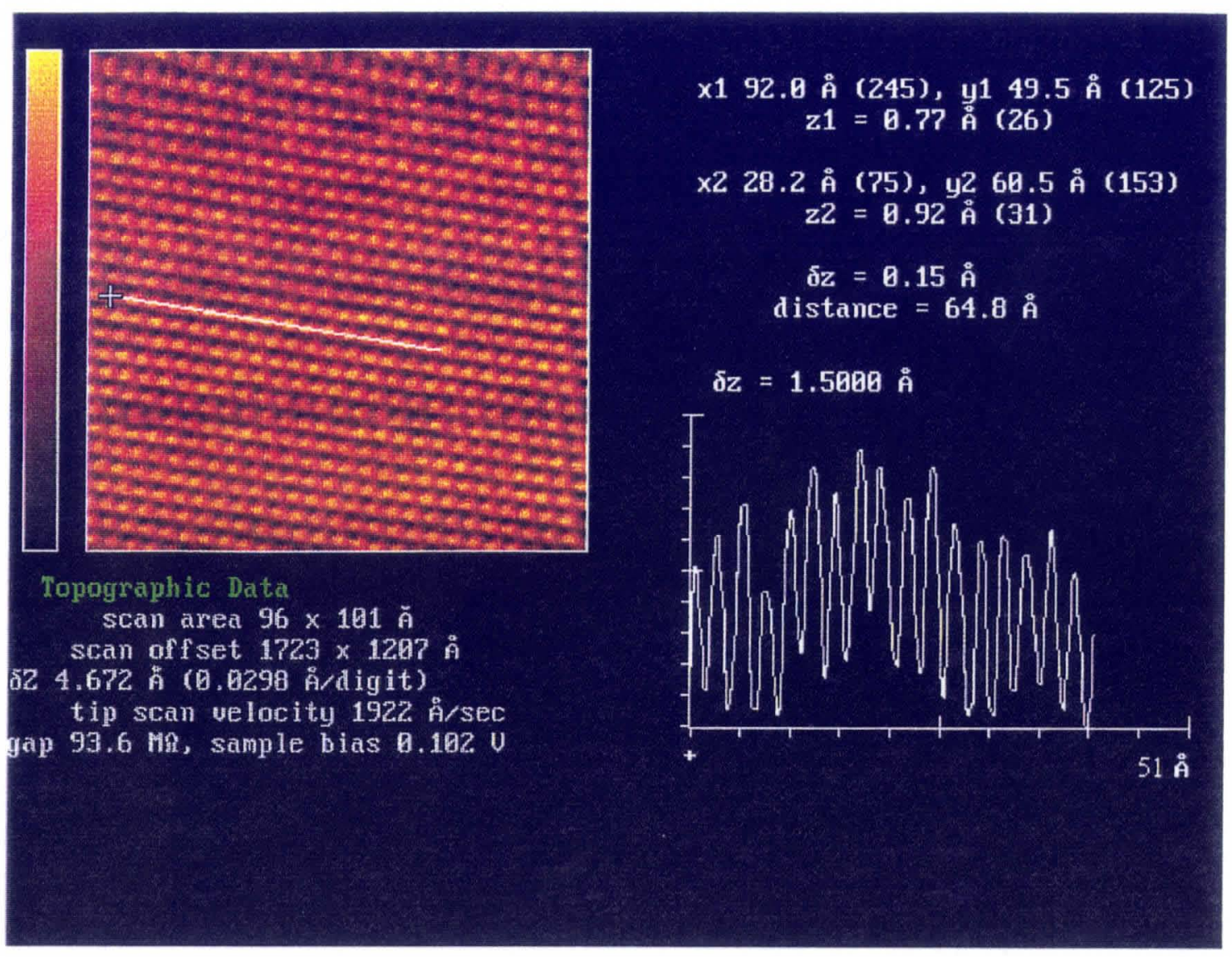

FIGURE 13. Calibrated cross section in the $x-y$ plane 
abundance of total charge values for data, he found that each of the total charges were divisible by a common denominator. This denominator was found to be the charge of the electron.

The generalization for the calibration of $\mathbf{z}$ scaling is certainly similar. By obtaining height measurements of features from topographic scaled images, one can determine the common factor for which each height is divisible. The vertical separations between graphite steps are used in this process. It was shown in FIGURE 11 that the separation between successive layers of this type of carbon is exactly $6.69 \AA$. The value would be the expected measurement for the common factor of heights. Furthermore, dividing the number of layers by this constant should yield integer values of layers. For example, a calculated result of $6.69 \AA$ is exactly two total layers, since every other layer is exactly the same. Any step separation distance found in the analysis which is inconsistent with the true factor will be corrected based on this result. Finally, the scaling on the topograph will be quantitatively correct when the vertical measurements are multiplied by the same correction factor.

The first image seen having stepped structure is illustrated in FIGURE 14. This two dimensional image depicts height variations by the contrast in color as seen on the color scale positioned left. Yellow represents higher elevation in contrast to lower elevation represented by the color orange. Hence, the image is a contour a step which decreases in elevation from left to right in a uniform direction.

Along side the topograph is a cross sectional plot. A cursor line drawn from the higher level to the lower level contains all of the data points for which the graph is taken. 


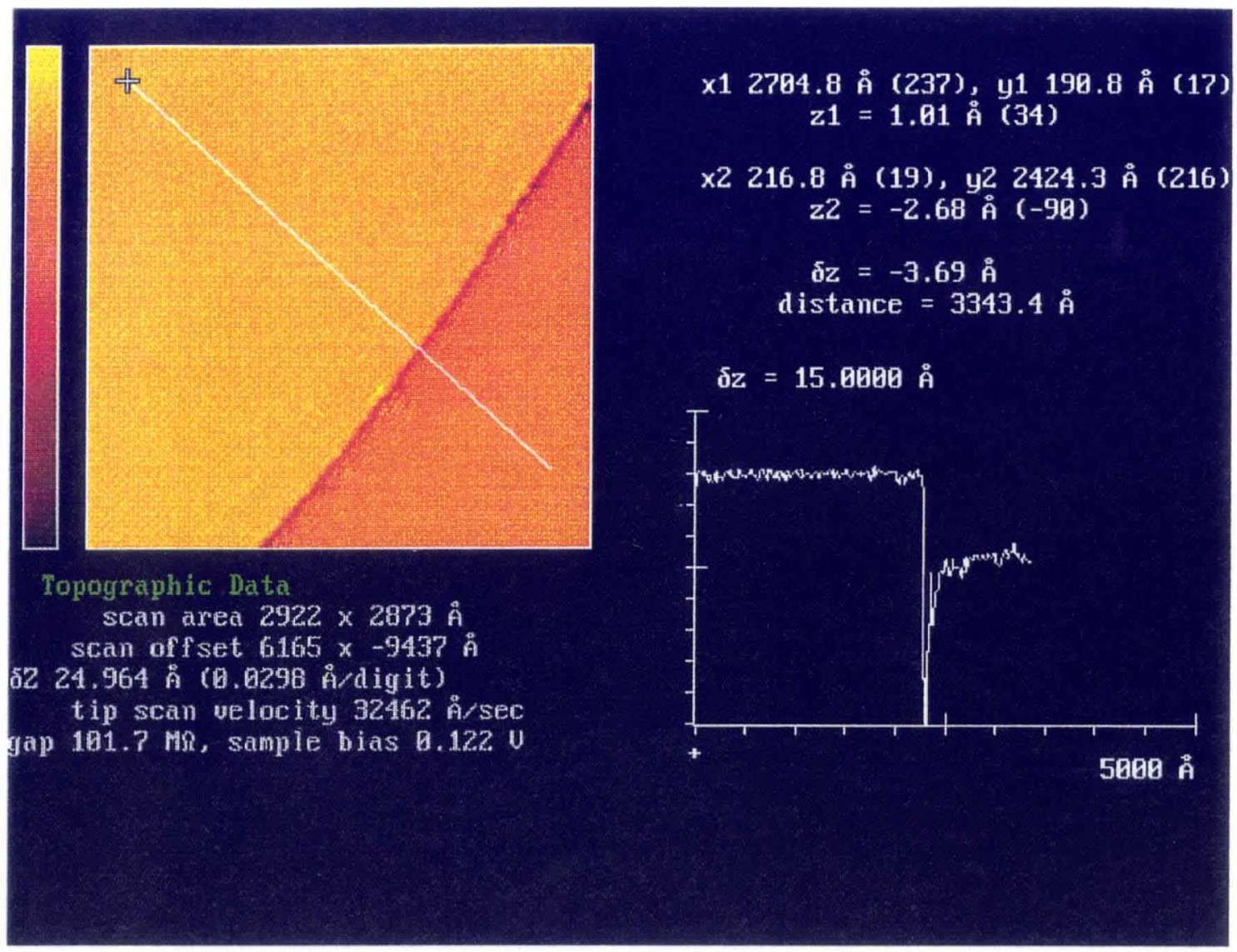

FIGURE 14. Uncalibrated 2D single step 
The data origin corresponds to the cursor position on the topograph. Experimental numerical data can be found both above and left of the graph. The scanning area for this image is shown to be $2922 \AA \times 2873 \AA$. An area of this magnitude contains more than one hundred thousand atoms. Therefore, only near atomic or "mesoscopic" resolution is permissible. The distance of separation between the two layers is given as $\delta z=3.69 \AA$. The value corresponds to the vertical position of atoms at each end of the cursor.

The general direction of the step is along the (110) vector using the Miller Index. From the information given regarding the cross section, the maximum scaled height $\delta z$ is $15.0000 \AA$. The software used suggests that the upper layer is separated less than 15.0000 $\AA$ from the lower surface.

There is one error in the cross section which is evident qualitatively. The lower surface should not have a defined height. A scanning tunneling microscope cannot measure the depth of any type of flat surface. Thus, the height measurement of the lower surface is fallacious. Consequently, the variation of the individual heights of the steps, according to the vertical scale, can be obtained by subtracting the scaled measure of the step height by the false height of the base of the sample.

A series of cross sectional plots is studied to obtain an accurate measure for the elevation difference. In the series, fifty $\delta z$ values are used for a statistical average. In this single step topograph the vertical separation for the step is $4.32 \pm 0.209 \AA$. As was previously stated this calculated value, divided by the layer separation constant $3.346 \AA$, should yield an integer value. The result of the division is a value of $1.29 \pm 0.088$ layers. The elevation change is therefore a single layer. In order for the scaling of the 
topographic data to be correct, the $\delta z$ value for the separation and scale distances must be multiplied by the correction value $0.774 \pm 0.053$.

If the relationship between the correction factor and the height of a step were not proportional, it would be evident in the analysis of different step heights. FIGURE 15 shows a two dimensional topograph of one such surface. The scanning area for the image is $2922 \AA \times 2873 \AA$. The distinct layers of the triple step can be easily identified by the color bands. Orange represents the highest elevation, and blue represents the lowest as is indicated by the color bar to the left of the image.

The cross sectional methodology for this triple step is the same as the single step. The cursor is initially positioned on the upper step initially. The vertical distance from this top layer to the lower layer is given as $\delta z=12.68 \AA$. The measurement is consistent with the cross sectional plot which scales vertically to $\delta z=15.0000 \AA$ and to $3000 \AA$ horizontally. Again, fifty measurements of the cross section are taken for this distance as well as for the separation between the middle step (red) and the lowest step (gray) to the bottom surface.

The average heights of the steps from the base of the structure is easily found. From the top step to the base the average distance is $13.05 \pm 0.2234 \AA$. The average number of layers is found by dividing this number by the layer constant $3.345 \AA$ is $3.900 \pm$ 0.0944 layers. The correct number of layers for this step should be three layers. Therefore, a factor of $0.7692 \pm 0.0186$ is needed for correction.

The average separation distance from the second step to the base is $7.56 \pm 0.402$ $\AA$. The mean layer result is $2.26 \pm 0.170$ layers. With the accepted separation being two 


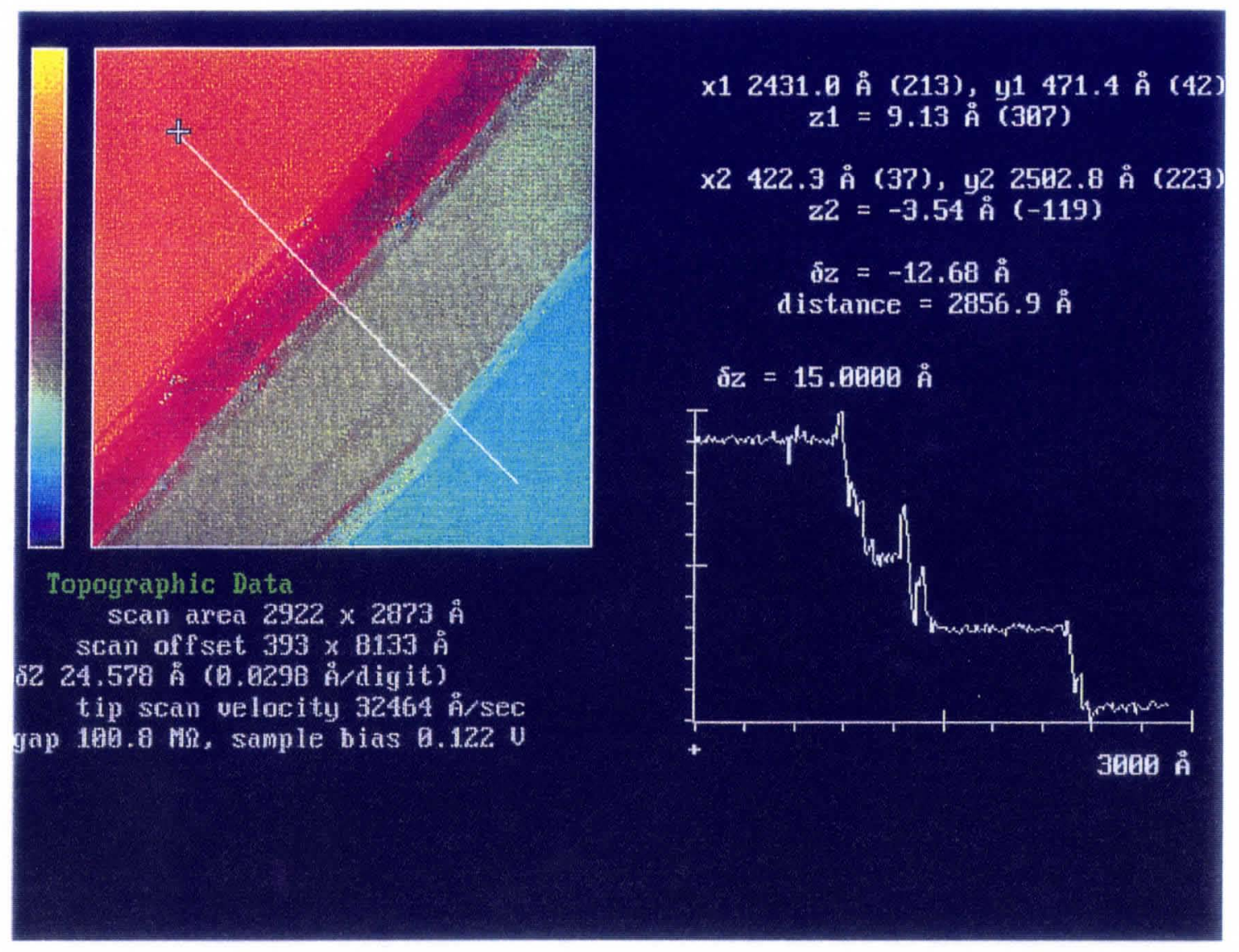

FIGURE 15. Uncalibrated 2D triple step I cross section 
layers, the factor $0.885 \pm 0.067$ is needed for correction. Similarly, the lowest step separation from the base of the triple step was found to be $3.90 \pm 0.397 \AA$. The resulting number of layers corresponding to this average is $1.17 \pm 0.168$ layers. At a single layer of separation, it is concluded that the appropriate correction factor is $0.858 \pm 0.125$.

A second triple step was imaged after scanning further. This is illustrated in FIGURE 16. The color bar is given showing the lighter color blue as higher elevation in contrast to the dark color blue, existing as the base of the triple step. The scanning area for this topograph is $2433 \AA$ x $2397 \AA$. Utilizing the same cross sectional methodology, an average of fifty height measurements are taken from the three different layers to the base of the structure. The separation between the top and the base is found to have a mean of $13.07 \pm 0.1804 \AA$. Dividing by the layer constant $3.345 \AA$ per layer results in an average of $3.908 \pm 0.076$ layers. The accepted value for the number of layers from the base of the sample is three for this step. The correction factor needed for calibration is then $0.7676 \pm$ 0.0498 .

The separation of the second step to the base is found to have a mean of $8.77 \pm$ $0.257 \AA$. The average step magnitude is determined to be $2.62 \pm 0.109$ layers. At two layers of separation, the mean correction factor $0.763 \pm 0.032$ is necessary.

Finally, the distance of separation between the lowest step and the base of the triple step is averaged to be $3.90 \pm 0.343 \AA$ corresponding to $1.16 \pm 0.145$ layers. The distance from the lowest step to the base is exactly one layer. The value $0.860 \pm 0.108$ is the accepted correction factor for the separation distance. 


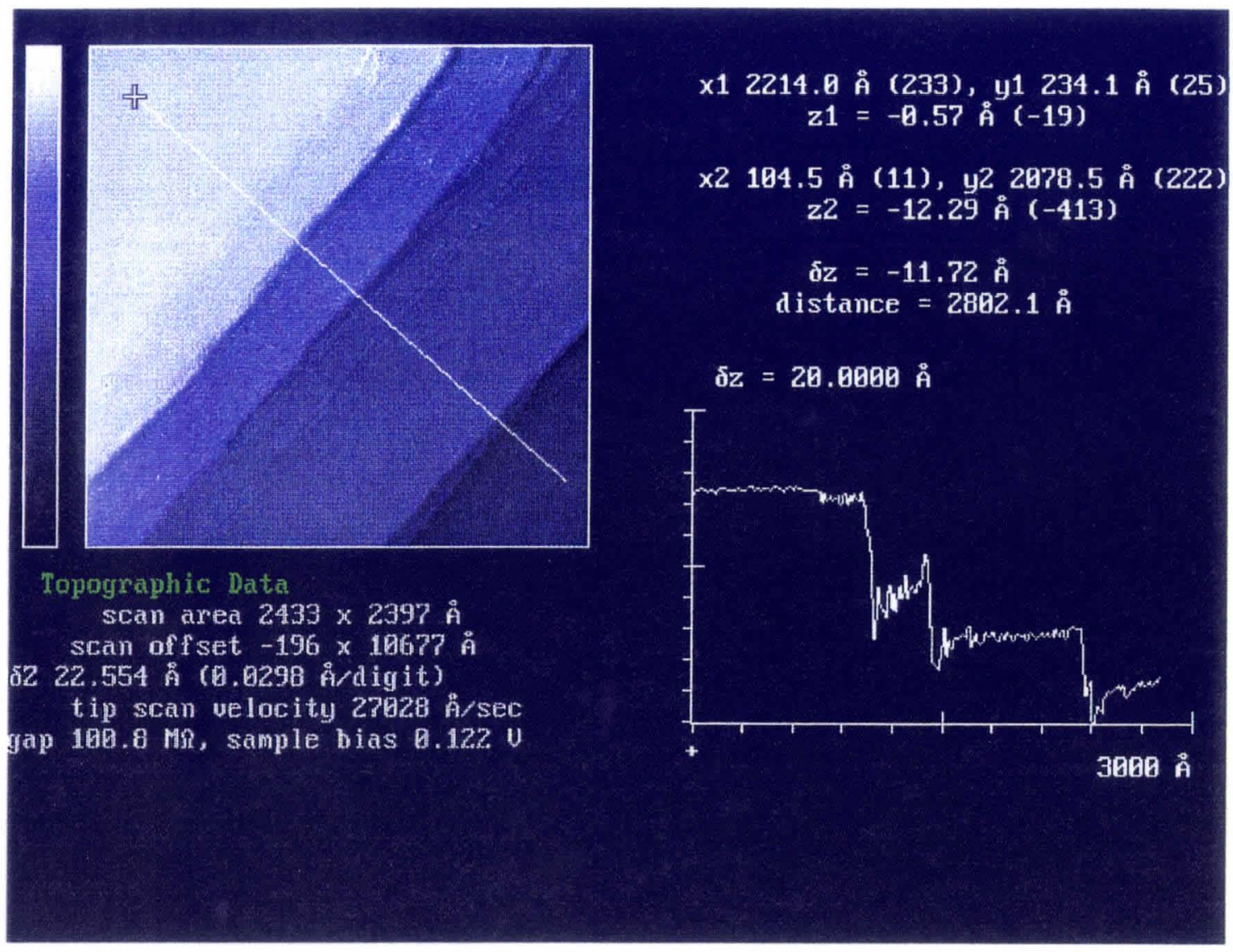

FIGURE 16. Uncalibrated 2D triple step II cross section 
The table below shows the averages of all correction factors for each of the three topographs and their respective step separations. The topograph of the single step has but one correction factor where as the two triple step topographs have three correction factors each.

$\begin{array}{cccc}\text { HEIGHT (Å) } & \text { FIGURE 14 FACTOR } & \text { FIGURE } 15 \text { FACTORS FIGURE } 16 \text { FACTORS } \\ 4.32 & 0.774 & - & - \\ 13.05 & - & 0.769 & - \\ 7.56 & - & 0.885 & - \\ 3.90 & - & 0.858 & - \\ 13.07 & - & - & 0.7676 \\ 8.77 & - & - & 0.763 \\ 3.90 & - & - & 0.860\end{array}$

A plot of these values is shown in GRAPH 3. Clearly, correction factors for topographic images produced by the STM are similar. Deviation accounts for some, but not all of the quantitative error in the graphs. There is another source of error which can be explained qualitatively.

The reaction time of the piezoelectric tube was insufficient to correctly depict the step structure. According to the cross section of the topograph in FIGURE 14, there is an extensive drop between layers. One may notice that there are also large pointed portions of the plot which occur near the boundaries. This is easily identifiable in a three dimensional figure of a triple step. The three dimensional image is illustrated in FIGURE 17. The dropping effect and the sharp protrusions are a result of an inadequate expansion of the PZT ceramic. If the creeping effect had not been present, the step structures would be strictly uniform. Since, however, they are present, we view these 
GRAPH 3. Correction factor versus step height plot

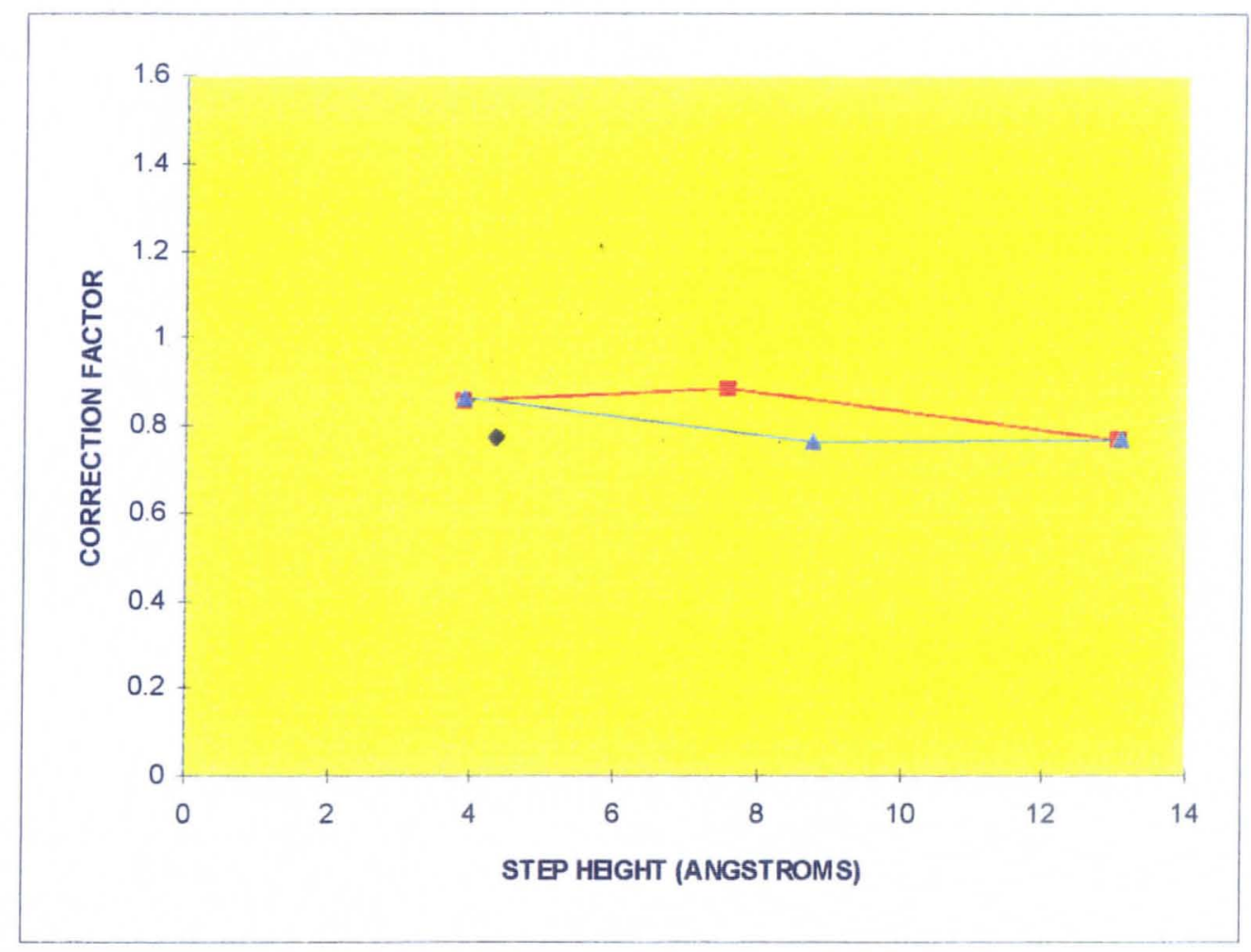

AVERAGE $=0.814 \pm 0.060$ 


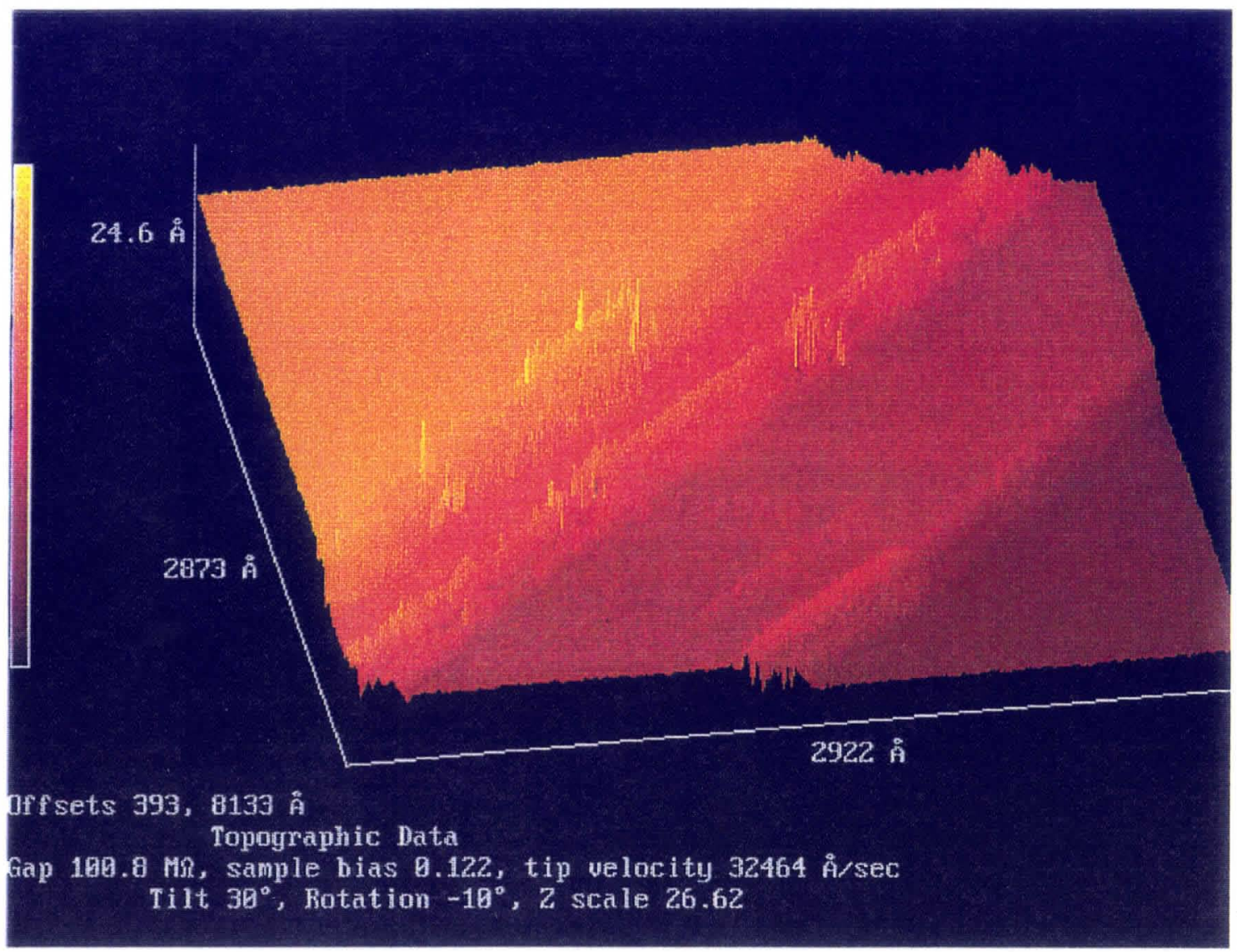

FIGURE 17. Uncalibrated 3D triple step I 
effects as a result of automatic changes in tunneling voltage. The voltage change accounts for piezo extensions during a constant current mode. Consequently, the average of the seven correction factors will set the linear limit on the final correction factor for the .topographic images found in this experiment. The limiting correction factor is found to be $F_{z}=0.814 \pm 0.060$.

This study then gives evidence that both horizontal and vertical measurements produced by the STM software STimage can be calibrated using a cross sectional method. Using the known distance between lattice sites will easily allow one to determine the calibration factor for horizontal directions. Equally important, an average of fifty $\delta z$ height measurements provides sufficient accuracy and precision to detail a correction factor for experimental image data in the vertical direction. One should always expect the division of this mean to be an integer number of the standard layer separation regardless of material. Clearly, knowing the structure of the sample is the key to understanding the methodology of these calibration techniques. 


\section{CHAPTER V}

\section{MULTIPLE TIP STRUCTURE : EXPERIMENT II}

\subsection{Structure Hypothesis}

Multiple tip imaging is a compound-tunneling phenomenon which usually occurs without welcome. Seeing disordered images on the monitor during scanning is not desired, since one usually has to retract the tip and electrochemically etch a new one. Each time this occurs it costs the researcher valuable time and effort. In the event that the researcher continues acquisition, he or she may find it difficult to differentiate between the alterations of topographs created by not having a single atom on a single apex. Also, it is important to be able to recognize features produced by a multiple tip which are not characteristic properties of the sample under study. The objective of this experiment is to identify the structure of a multiple tip using only topographic images. Multiple tips are found commonly, though to seek to produce one, trial and error of etching and scanning is unavoidable.

A graphite sample will be used in this study with a scanning surface area containing a crash hole sight. Holes can be made by making direct contact between the tip and the sample. After contact has been made, the tip is discarded and replaced by a new electrochemically etched tip. The sample surface is then carefully recleaved without removing the crash hole sight and repositioned in the sample apparatus. 


\subsection{Structure Review}

Research has indicated several different methods for determining the structure of a multiple tip. It is the purpose of this section to discuss those techniques. It may then be possible to utilize one or more of the examined approaches to determine the structure of the multiple tip in this system.

Paik, Kim, and Schuller suggest that the apex structure of the tip cannot be determined during experimentation, for the tip can be easily altered during scanning motion. ${ }^{27}$ It is possible, however, to determine the structure after scanning topographs in Atomic Force Microscopy (AFM) have been brought forth. This is accomplished by studying the modification of images that results from different tip structures and orientations. ${ }^{27}$ The group has examined triangular, rhombic, and seven atom hexagonal structured multi-tips for their unique density dependent amplitude and phase shift plots, which are a function of the tip orientation. ${ }^{27}$ It is seen that as the diameter of the tip or the angular orientation of tip structures increases the width and amplitude peaks decrease. ${ }^{27}$

If the orientation plots are used, one must first achieve atomic resolution with a single-atom tip. When multiple tips occur, the topographs can then be compared to the models the group has presented. Such an analysis can only be conducted with samples containing solid structures known prior to experiment. A good example of this is graphite. Graphite samples exhibit lattice rows, triangular arrays, and also honeycomb shaped formations. It is mentioned that for a specific double tip structure of platinum, when rotated through $\pi$ / 12 radians with respect to the $\mathbf{z}$-axis, the geometric image amplitudes will decrease. ${ }^{27}$ The resulting change for a sample of graphite is a honeycomb 
shape changing to a triangular array of ellipses to finally an image of lines. ${ }^{27}$ The geometrical structure can indeed be determined in this manor, considering that different atomic tips will have different resulting changes. Characteristics of nickel $(\mathrm{Ni})$, platinum, and tungsten tips are presented in their formalism.

A method presented by Sasaki and Tsukada is similar to the one previously discussed. $^{28}$ Also using AFM, the researchers state that orientation studies of multiple tip structure can help one to determine the structure of a sample which is unknown. ${ }^{28}$ Obviously, by using the same multiple tip to scan carbon-graphite and unknown surfaces, one can compare the topographs presented by each and determine the structure of the unknown sample.

In the interest of tip structure determination, like Paik's group they also consider effects of sample images made by altering the orientation, though images they observe are scanned only by carbon-diamond tips. Orientation plots and images of a graphite sample influence the conclusion that as one changes the structure of the tip in length at the apex, the topographic structures can be found to change. ${ }^{28}$ Thus, one can use topographs of graphite to similarly determine the structure of diamond multiple tips. ${ }^{28}$

Multiple tips are common occurrences to both AFM and STM experiments. STM researchers Park, Nogami, Mizes, and Quate have seen this problem more than most scientists in the community. ${ }^{29}$ These specialists have found that when a doubling of the number of atoms on the surface of the sample is illustrated on topographs, the scanning probe consists of a double tip structure. ${ }^{29}$ They have published images of silicon taken during acquisition. For this case, the contribution of tunneling intensity from the two tips 
is comparable. ${ }^{29}$ The doubling, however, is asymmetric on some of the plots. ${ }^{29}$ That is, doubling is present on half of the image and absent from the other half. They attribute the asymmetry to one of the tip asperities being closer than the other to the sample. ${ }^{29}$ Hence, one of the asperities moves out of the way during acquisition.

Further analysis showed that the effects of multiple tip imaging are voltage dependent. ${ }^{29}$ The arrangement of atoms exhibited by the double tip was different when reversing the polarity of the tip to negative and the sample polarity to positive ${ }^{29}$ When the bias is opposite, the electrons are tunneling from the sample to the tip. The difference in the images attained was found to be the result of the two containing two different atoms. ${ }^{29}$ One of the atoms on the tip was found to be a surface atom of silicon picked up sometime during scanning motion. It was agreed that if the two elements were both silicon or both tungsten, then the dependence on bias polarity would be the same. In this case, the change of the topographs would not be existent.

Along with Albrecht, the group continued their multiple tip research on graphite surfaces ${ }^{30}$ Moire patterns were observed in images where the interpretation of the top layer gave the appearance of being spliced and partially reoriented. ${ }^{30}$ The orientation mismatch occurs at angles of $6.5^{\circ}, 8.0^{\circ}$, and $19.0^{\circ}$ based on the known hexagonal lattice structure. $^{30}$

A solution proposed by the researchers indicating the mechanism which causes such mapping was one tip scans a particular realm while the other scans another realm. ${ }^{30}$ The superposition is distinctly similar to that of the images seen in the previous study. The 
region of orientation in the Moiré patterns was determined to set the limit of minimal separation between the double tips, on the order of $100 \AA^{30}$

Klitsner, Becker, and Vickers used a tungsten wire in their scanning tunneling microscope to observe topographical image changes from germanium by the dual polarity technique. ${ }^{31}$ The images were analyzed while sweeping the tunneling voltage from $-2 \mathrm{~V}$ to $+2 \mathrm{~V} .^{31}$ It was apparent that protrusions were visible in scans of negative bias, where electrons travel from sample to tip. ${ }^{31}$ The aspect, however, was seemingly unpresent in scans of positive bias, where electrons go from the tip to the sample. ${ }^{31}$

Examination of the phenomenon leads to the conclusion that the protrusion was the result of a double tip. The occupied state density topograph was increased in magnitude which detailed the protrusion defects. ${ }^{31}$ Using a reverse tunneling bias of -1.8 $\mathrm{V}$ the structure of the tip is projected onto the topograph. ${ }^{31}$ The image depicts a large protrusion of height $2.3 \AA .^{31}$ The measured height of the second protrusion was $1.9 \AA^{31}$ Cross sectional analysis showed that the central separation of the two protrusions was exactly $9 \AA^{31}$ The final conclusion the three researchers made was the taller peak was formed by the tip that was closest to the sample. ${ }^{31}$ Conversely, the shorter protrusion corresponds to the tip which scanned furthest from the gallium sample. ${ }^{31}$ The variation in height was found to be the distance from the end of the tungsten wire to the lower protrusion. This distance was determined to be $0.4 \AA .^{31}$

Loenan, Dijkkamp, Hoevan, Lenssinck, and Dielman studied silicon with an electrochemically etched tungsten wire. ${ }^{32}$ In the experiment, contact was made between the tip and the sample by direct insertion. Repetition of contact while changing the lateral 
position of the tip resulted in the production of a linear indentation. ${ }^{32}$ When the tip was extracted, the image was found to depict two identical lines instead of just one. ${ }^{32}$ The conclusion was the apex of the probe contained a double tip. ${ }^{32}$ Cross sectional analysis perpendicular to the direction of the indentation showed the ghost image is spaced at a distance of $8 \mathrm{~nm}$ from the real indentation. ${ }^{32}$ Double tip spacing was determined to be the distance from the trough of one indention to the other. The magnitude of the distance was exactly $8 \mathrm{~nm} \cdot{ }^{32}$ Further investigation was conducted to explain other features projected on the STM topograph.

On the same topograph with the indentations, four protruding structures of similar needle-like form were also visible. ${ }^{32}$ The placement of the structural pairs was solitary. One pair was fixed on the left and one was fixed on the right. ${ }^{32}$ Height variation plots were guided over these two separate sets. The profiles indicated that the structures on the left were of different height, yet both had double protrusions. ${ }^{32}$ For the structures on the right, the same characterization applied, but the widths and lengths of the structures were greater than those of the left hand side. In each case, the interrelated structures were exactly $8 \mathrm{~nm}$ apart. $^{32}$

The cross section of the smaller structures appeared to have the same shape as the indention cross section would have if it was simply turned upside down. ${ }^{32}$ Since the indentation profile matched the protrusion profile inversely, the ghostly structures must play a part in describing the form of the double tip. It was mentioned that each ghost structure had double protrusions. ${ }^{32}$ The protrusions of the left were far less pronounced than those of the right images. In response to this, the deduced cause is a double tip 
having one apex closer to the sample than the other. ${ }^{32}$ The longer tip contributed the most to current. This is visible in the left ghost images. During rastering movement, the swinging effect brings the lower apex closer and the higher apex further away. ${ }^{32}$ The result is a more equivalent unoccupied state density plot.

\subsection{Structure Experiment}

The second experiment undertaken in this thesis is devoted to determining the structure of a multiple tip based on topographic images. Connections and operations of the scanning tunneling microscope have been discussed in detail already. The images are attained using STimage software and a model 100 STM system.

Research has indicated that distinct structures on the surface of a sample with known form may assist the observer in identifying false projections. It is for this very reason that a crash hole was made. As the tungsten tip comes slightly into contact with the graphite sample, it generates an imprint of the shape of the tip. The smooth and resolved lattice structure of the carbon around the cavity insures that multiple tip effects will be noticed. It is impossible to accurately predict the arrangement of atoms on the apex of a tungsten wire from the electrochemical etching process. Consequently, tunneling probes had to be re-etched and tested in the system until imperfections were imaged.

Defects on images would verify that the system was infected by multiple tip construction. Therefore, it is a trivial task to analyze the alterations encountered during the experiment. 
With the probe in range of the sample and stable in a constant current mode of $1 \mathrm{nA}$, the first image is resolved. FIGURE 18 is a two dimensional topograph for the initial structures seen. The indentation made by the tip is clearly visible. Lattice resolution in the $1478 \AA \times 1459 \AA$ scanning area containing the aperture is observable in zoom as well. This appearance of the cavity confers a foot shaped pattern. The overall length is $1313 \pm 0.5 \AA$ according to scale. From this data alone, it is very possible that the probe's position was displaced as it crept into the sample. The view is better seen by the three dimensional topograph in FIGURE 19. Further scanning proves this hypothesis to be improbable. The three dimensional topograph in FIGURE 20 indicates the cavity is rather circular in form. A sizable lip on the edge and angled inclination of the hole allows one to speculate that the tip was rastering towards the left when contact was initialized.

A mysterious occurrence developed when the scanning continued. The unoccupied state plots exhibit cavity diameter changes over different scans. The first changes observed were slight. The cross section given in FIGURE 21 yields a length of $609 \pm 0.5 \AA$. The cross section in FIGURE 22 shows the distance to be $703 \pm 0.5 \AA$. Between two scans, there was a difference of $94 \pm 0.5 \AA$ in measurement for the hole's diameter. The change persisted as raster scanning continued. Since the bias is not high enough to alter the structure of the sample at $0.123 \mathrm{~V}$, the multiple tip effect is conclusive. ${ }^{33}$ A threshold potential of $3.25 \mathrm{~V}$ would be needed to do so. ${ }^{33}$

This occurrence developed into more unusual peculiarities. Exhibited in FIGURE 23 , there appears a shadow of similar size and shape aside the hole. Inspection of the 


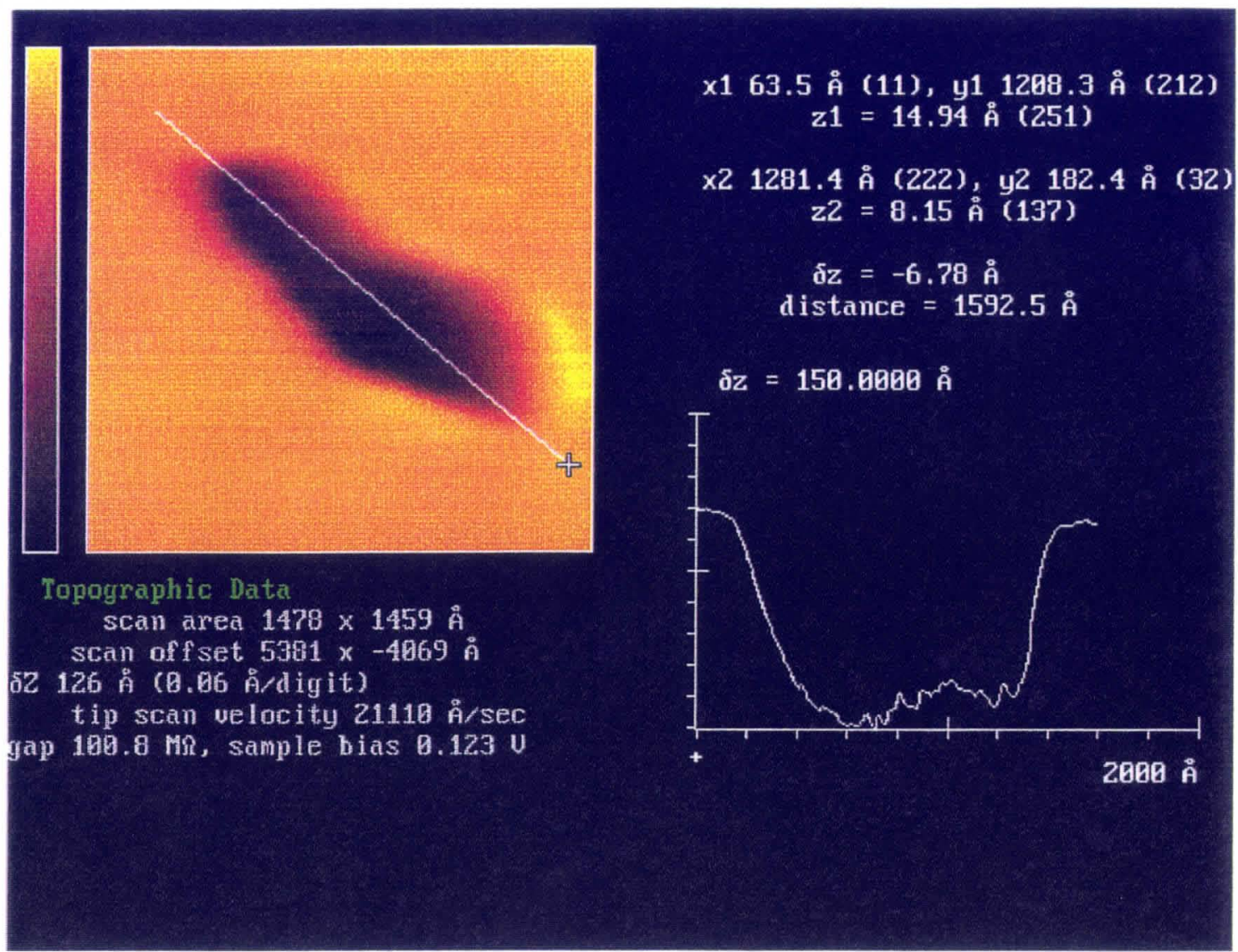

FIGURE 18. 2D 1313 angstrom wide crash hole sight first seen 


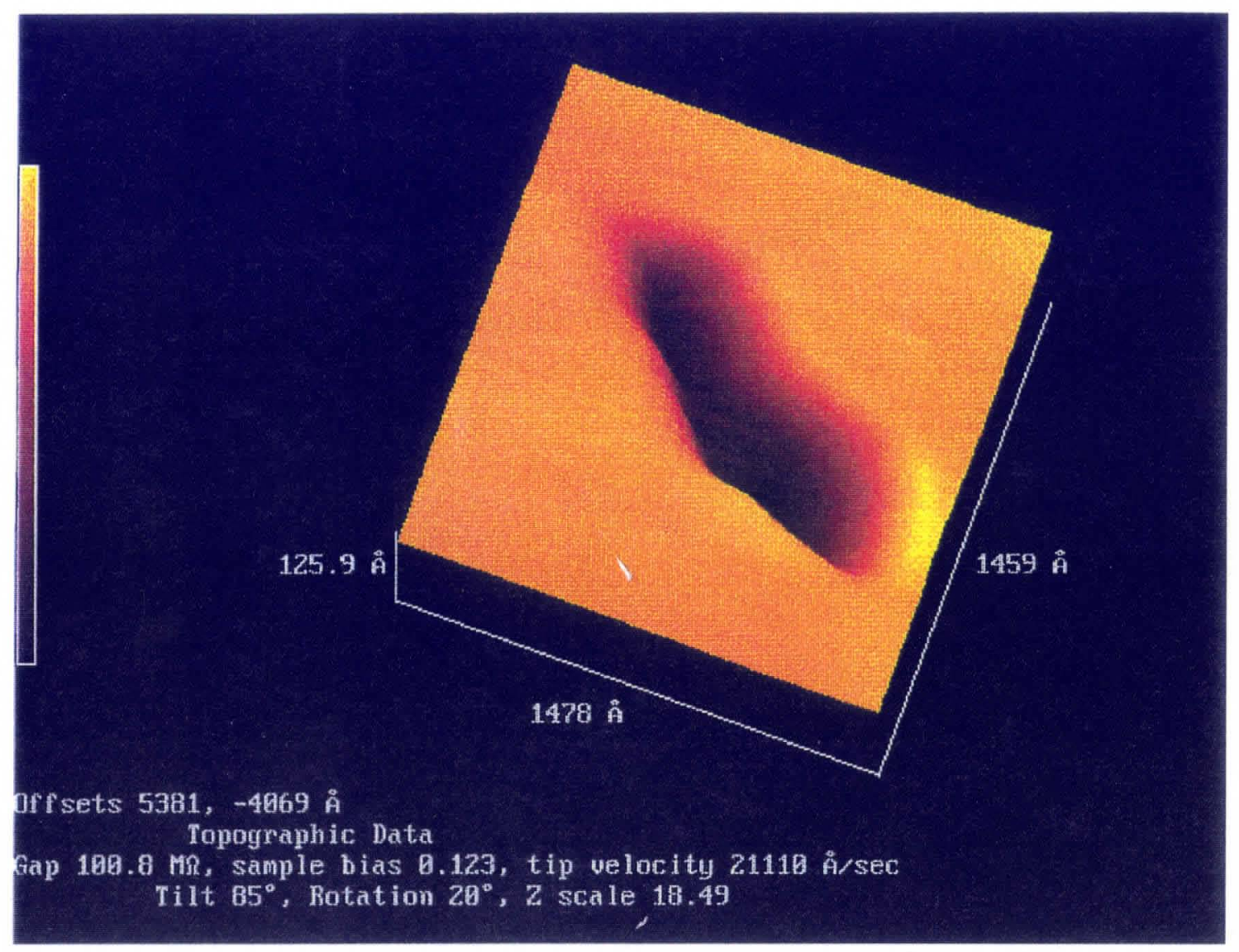

FIGURE 19. 3D 1313 angstrom wide crash hole sight first seen 


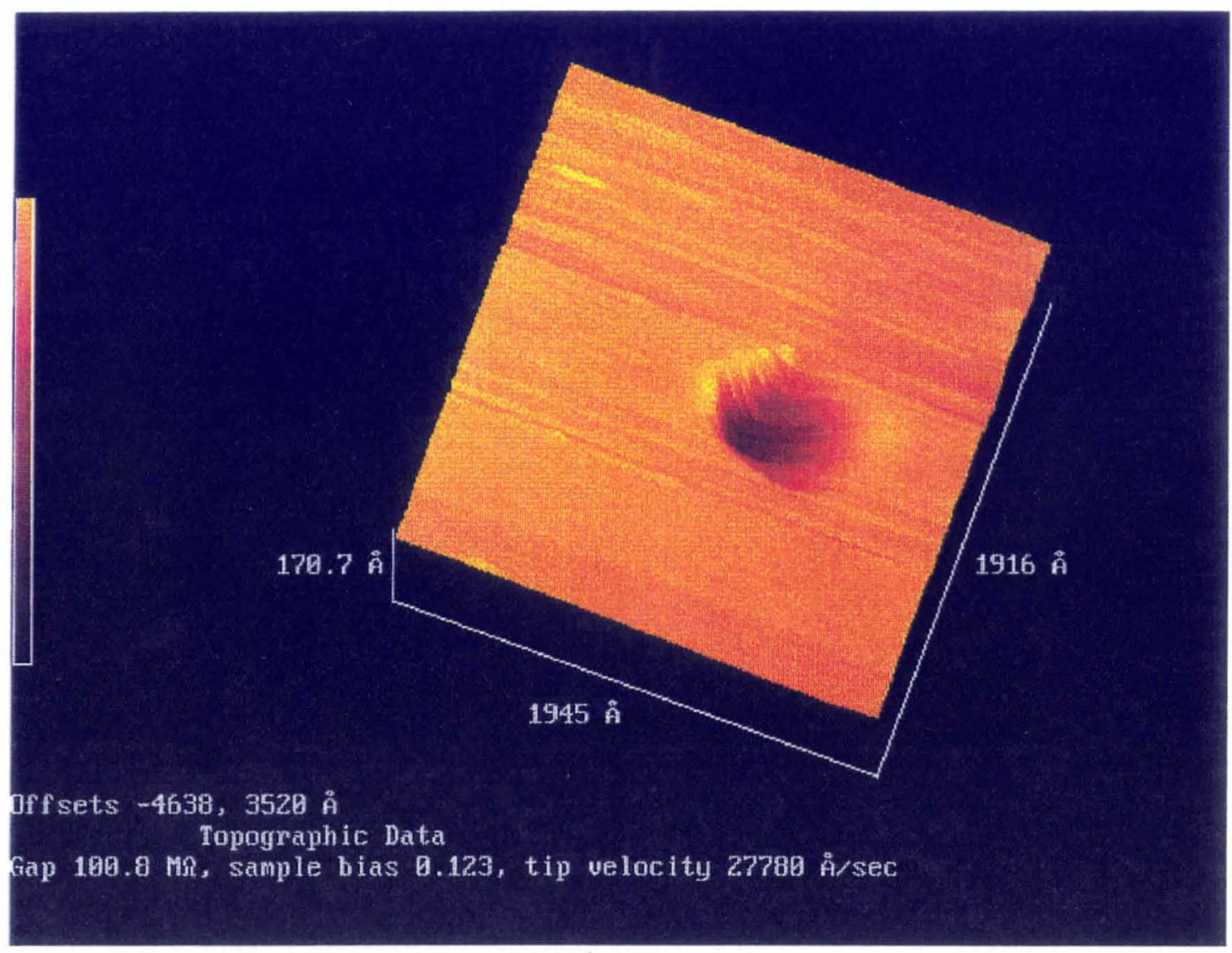

FIGURE 20. Circular cavity change is visible 


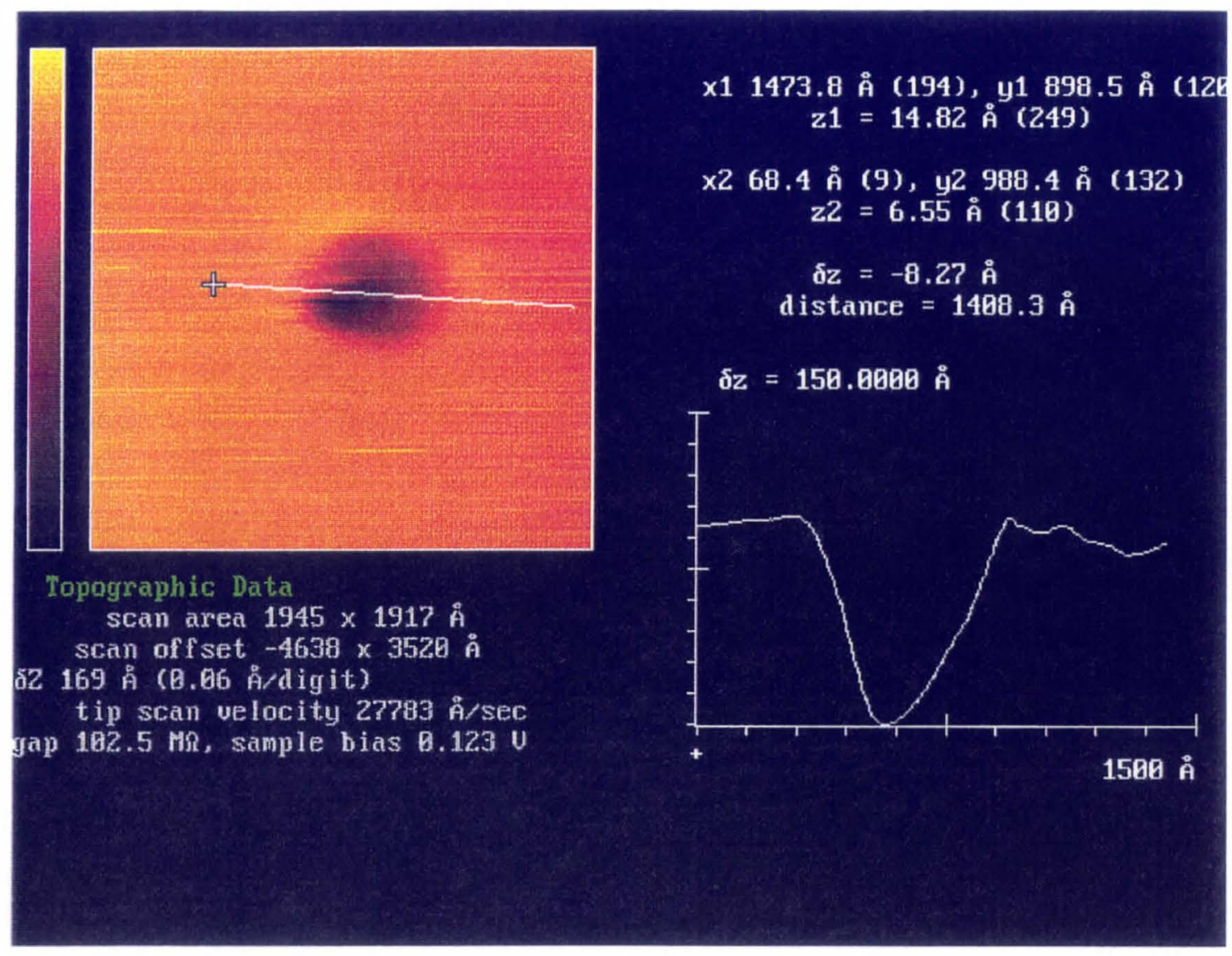

FIGURE 21. 609 angstrom wide cavity 


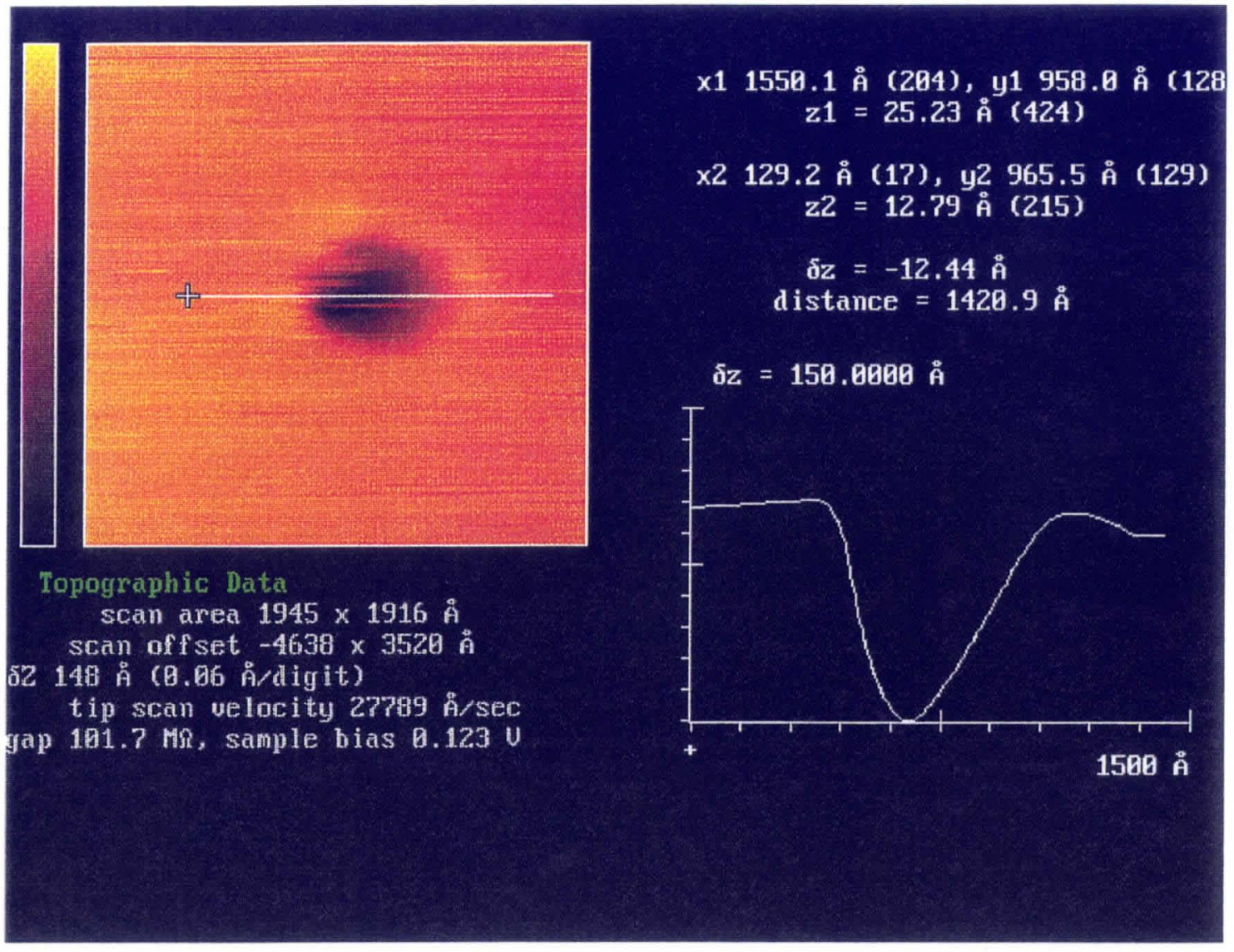

FIGURE 22. 703 angstrom wide cavity 


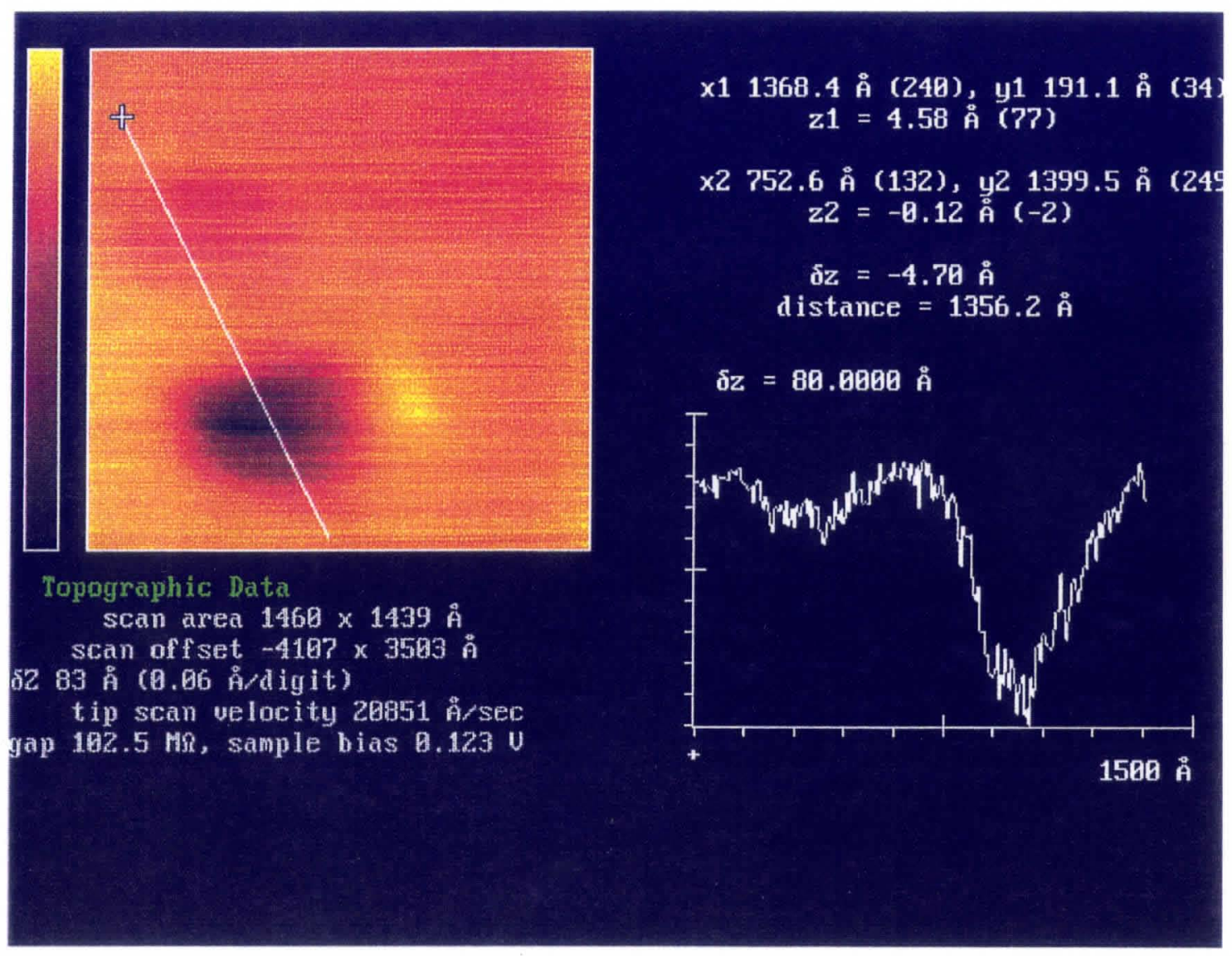

FIGURE 23. 703 angstrom wide initial hole with a 469 angstrom wide shadow 
amplitude variation results in finding an original indention which is $656 \pm 0.5 \AA$ in diameter and a smaller shadow indentation of diameter $469 \pm 0.5 \AA$. This dimension changes for each in the next scan ( see FIGURE 24). The initial cavity is momentarily 563 $\pm 0.5 \AA$ in width. The cross section of the newer cavity has a measurement now greater than the initial cavity at $591 \pm 0.5 \AA$. The change in form is not the only visible activity in the plots. The distance from the new cavity to the original site increases with more scans. The span is never found to decrease.

The shadowing nature is similar to the effects seen by Loenan's group. ${ }^{32}$ There are definite similarities with the puncture cavity's shadow illustrated in these topographs in comparison to their duplicate inscription lines on silicon. The twofold, unoccupied state images resemble the images studied by Klitsner's retinue. ${ }^{31}$ The height variations of both positive bias cross sections reveal a large dip and a smaller dip. It is conceivable for each of these cases that the compound tunneling effect was the result of the probe containing a double tip. It is likewise convincing that a double tip exists in this experiment as well. None of the research previously detailed, however, explains the nature of image pairs altering their spacing during acquisition.

On the point of continuation, the span between the new cavity and the original cavity ceased to change. Still, the diameter of the indentation was changing. If the diameter increased, the relative depth of the cavity increased as well. Conversely, the depth decreased with the diameter. Changes in depth can be seen from the unoccupied state plots. The hole on the left increases its depth in each scan to follow. As this cavity 




FIGURE 24. 563 angstrom wide initial hole with a 591 angstrom wide shadow 
becomes more developed, the original one mutates to a more depthless form. The transformation of these contours are implicitly visible in topographs displayed in FIGURES 23-25.

The theoretical cause of the shadow reproduction was made less complex by previous researchers. It is indisputable that the probe is scanning with a double tip. It is enlightening that some research has concluded whether or not the tips were equidistant on the probe. Furthermore, some have identified that the tips contribute to current differently, as a result of having unlike separation gaps. There is a resulting comparability in tunneling current when this happens. Hence, there will exist a superposition of electronic wavefunctions from the two sites, not to mention an image doubling. The interesting characteristic of those experiments is that the tips were stationary during scans. In contrast to discoveries from all previous researchers, the configuration of the tips is not stable on the apex of the probe during acquisition for this experiment.

Between all of the topographs presented, one can visualize the phenomenon as a cavity transforming slowly into another cavity. FIGURE 23 and FIGURE 24 introduce this transformation and yet another conspicuous phenomenon: the distance between the double tips increased between scans. A new conclusion can be drawn as to state that an atom or atoms at the apex of the furthermost tip moved during rastering motion. The distance traversed was so great that this asperity stopped at a distance closer to the sample than the original tip. 


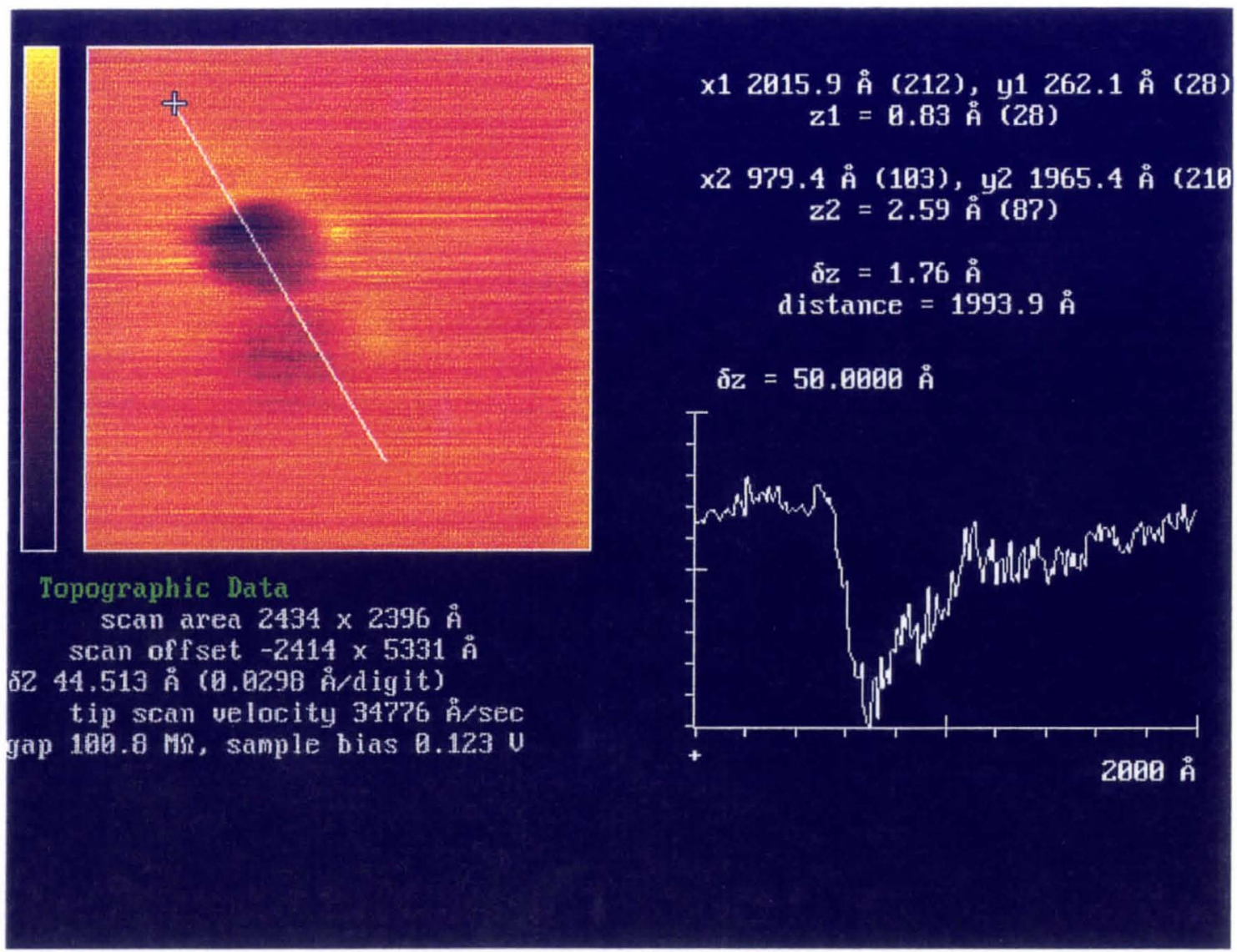

FIGURE 25. Shadow hole has transformed almost completely 
Separate studies previously discussed have indicated schemes of ascertaining the structures of their double tip probe ${ }^{29,31,32}$ A limit to the span can be set by resolving the distance of separation by cross sectional analysis. From the cross sectional topograph of FIGURE 24, the representative final location of the asperity displacement is the distance between the two troughs. This length is measured to be $628 \pm 0.5 \AA$. Tip heights were determined in a previous study by analyzing occupied state density plots for a double tip protrusion. ${ }^{31}$ The occupied plots looked just like the inverse of the unoccupied plots. It is then feasible to utilize the positive bias cross sections to indicate the length of the tips relative to each other. The vertical separation is $4.2 \pm 0.5 \AA$ between the two tips. Looking back at FIGURE 23, the tip to tip separation was $703 \pm 0.5 \AA$, and the vertical separation was $52 \pm 0.5 \AA$. It is remarkable that such a change can develop while imaging only a few times.

Most of the calibration possibilities for these images are impossible to achieve here. There is never atomic site resolution with large scanning areas of the order $2000 \AA x$ $2000 \AA$. With no atoms in view to utilize the cross sectional calibration method, any attempt to calibrate in the $\mathbf{x}-\mathbf{y}$ plane is impossible. The order to which measurements along this (001) direction are significant and can, however, be validated without calibration.

Based on the images retrieved in this experiment, it is conclusive that the phenomenon seen is a result of double tip imaging. Furthermore, the distance between the two tips was found to increase to $703 \pm 0.5 \AA$ during scans. The vertical separation 
between the original cavity and the shadow cavity change significantly as well. Originally, a single crash hole was imaged. The nature of the topographs depicting the presence of a new hole as a result of a second protrusion on the apex of the tip displacing its position on the tip. As such, the shadow is caused by a protrusion moving close enough to the graphite sample for tunneling to occur. When the cross section showed a vertical depth which is greater for the shadow hole, the extra protrusion moved closer to the sample than the original tip. When one tip is extremely close to the sample, for example the rightmost tip, the rightmost hole is much deeper than the leftmost hole. The converse is true when the leftmost tip is closer. When the distances of the tips are in near equilibrium the depths become shallow.

It is probable that the minute change in diameter during scanning is a result of inappropriate scaling. The voltage supplied for this experiment was not high enough for sample alteration. Hence, the width of the crash hole should not have been modified in any event.

With a conical shape pointing towards the sample, the extra protrusion should have came closer to the initial tip while scanning. Topographs illustrate this was not the case. The ghost hole was perceived to move away from the initial hole. A conclusion is drawn now to say that the two tips were coupled beforehand. As time continued a dwarfed form resulted. An abundance of atoms on the end of the tip were reoriented by contact caused by creeping or hysteresis of the piezoelectric tube. Atoms of the graphite were picked up from the tip while altering the position of tungsten atoms of the tip. Tungsten atoms were left on the sample surface. The rastering motion of the tip and the 
electric field were the basis for the phenomenon occurring then: atoms of the separated protrusion migrated away from the sample and up the side of the tip. FIGURE 26 shows this change during scanning.

One should keep in mind that the crash hole was produced in an experiment beforehand intentionally. The migration activity of atoms on the tip is indeed unexpected. Research indicating such double tip motion has not been discovered. It is only now that STM and AFM scientists will be able to clearly introduce the effect of image movement caused by atomic migration on the surface of the tip. In addition, double tip imaging defects have never been seen at distances of the order $500 \AA$. In this experiment we have seen the effect of a double tip at roughly $703 \AA$ in separation. 


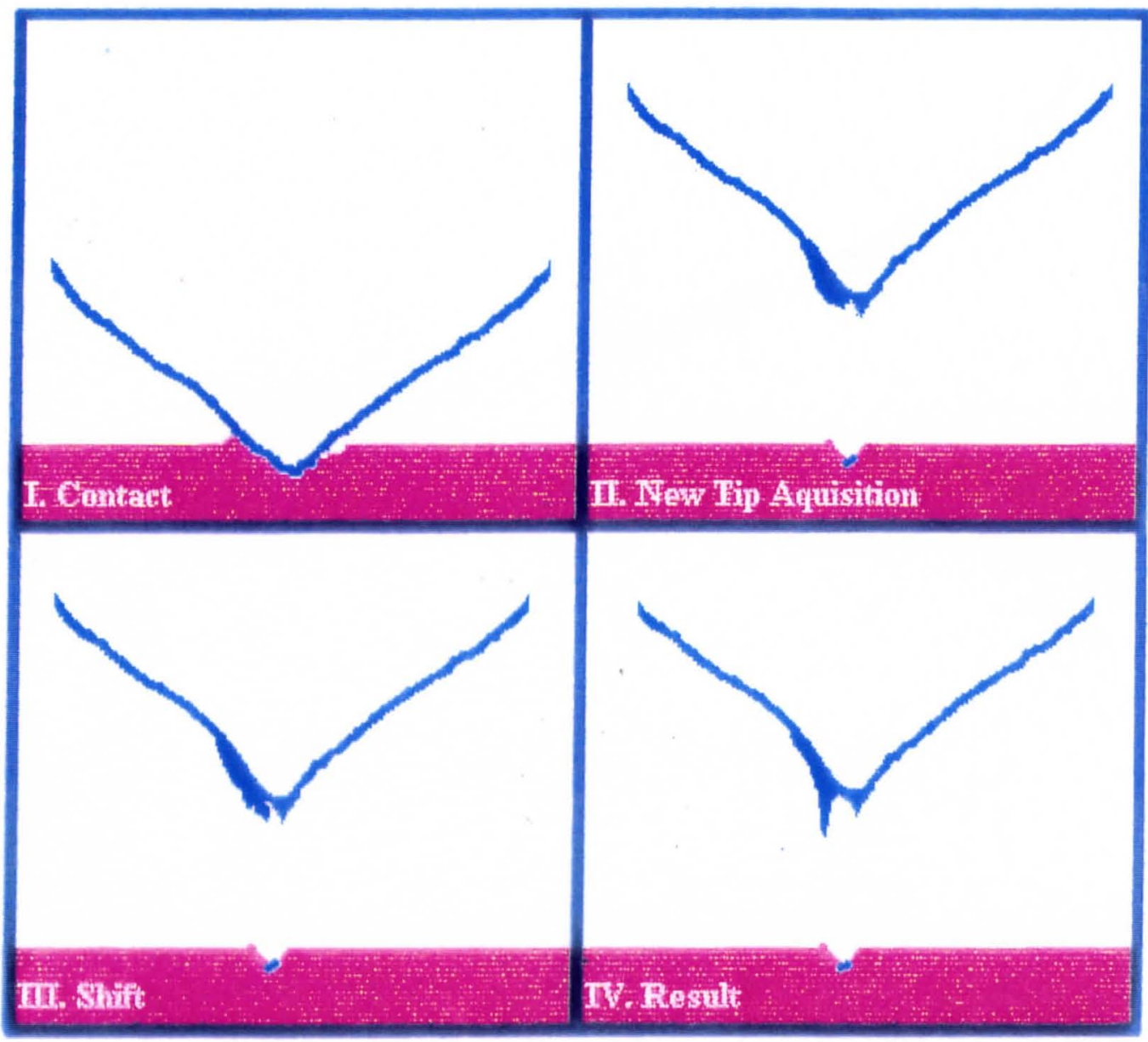

FIGURE 26. Transformation depiction for the double-tip 


\section{CHAPTER VI}

\section{RESULTS AND DISCUSSION}

\subsection{Analysis Conclusions}

Calibration techniques must be conducted separately for $\mathbf{x}-\mathbf{y}$ plane and $\mathbf{z}$ axial data. The simplest way to calibrate scaling in Miller planes (100) and (010) is to utilize the lattice constant for B-B atom separation. The distance between any two B atoms should be the distance between amplitudes of a cross sectional contour along the lattice direction. This distance is exactly $2.46 \AA$ for carbon-graphite.

For calibrations in the Miller plane (001), multiple layered structures such as steps provide the best data. The distance from lower levels must be an integer number of layers from the ground level. The layer separation constant $3.345 \AA$ should be used when dealing with graphite. As was the case with STimage, alternative types of software will also provide faulty scaling in the $\mathbf{z}$ direction. In the case that a linear or proportional relationship is not feasible for calibration, it would be worthwhile to determine a standard function by which one could adjust analytical topograph data.

In the calibration experiment, it was found that the correction factor needed to properly calibrate the $x-y$ plane was $F_{x, y}=0.64 \pm 0.03$. The limiting correction factor found for scaling normal to the sample surface is $F_{z}=0.814 \pm 0.060$. It is apparent from the previous studies that the analytical data is accurate to nearly $64 \%$ parallel to the sample and $81 \%$ orthogonally. Such calibration techniques should become very useful for STM and AFM researchers. 
The objective of determining the structure of a multiple-tip was fulfilled. The finding of a double-tip of separation $703 \AA$ was unexpected. Rare research is available on such widely spaced apii. Scientists should review the studies included to privide insight for image defects. Focus on the defects of topographic data have been researched, but in minor detail. Still, the findings only touch the scope of experimental thoery in multiple-tip imaging. It is conclusive from this research that images which illustrate duplication of sample structures are a result of double-tip imaging. It predicts the possibility that a triple-tip would be the cause of structural images in triplicate and so on. The newest finding is sample movement. The reformation of atomic sites have never before been seen under a STM when scanning by double-tip. An an STM novice, this physicist hopes that other defects such as these are studied and introduced to the surface science community. 


\section{REFERENCES}

G. Binnig, H. Rohrer, C. Gerber, and E. Weibel, Phys. Rev. Lett. 49, 57 (1982).

A. Cricenti, S. Selci, A. C. Felici, R. Generosi, E. Gori, W. Djaczenko, G. Chiarotti, Science 245, 1226 (1989).

David Bohm, Quantum Thoery (Prentice-Hall, N.J., 1951), pp. 264-266.

C. B. Duke, Solid State Physics: Tunneling in Solids (Academic Press, N.Y., 1969), Sup. 10, pp. 34-58.

John G. Simmons, J. Appl. Phys. 34, 1793-1800 (1963).

J. Frenkel, Phys. Rev. 36, 1604-1618 (1930).

N. Mingo, L. Jurczyszayn, F. J. Garcia-Vidal, R. Saiz-Pardo, P. L. de Andres, F.

Flores, S. Y. Wu, and W. More, Phys. Rev. B 54, 2225 (1996).

D. R. Lide, CRC Handbook of Chemistry and Physics (CRC Press, Fl, 1994), 75th ed., pp. (4-74), (8-23), (12-26, 113, 114).

Chunli Bai, Scanning Tunneling Microscopy and its Applications (Springer-

Verlag, N. Y., 1995), Ser. 32, pp. 15, 36.

Dag 155, Acheson, Port Huron, MI.

J. P. Ibe, P. P. Bey Jr., S. L. Brandow, R. A. Brizzolara, N. A. Burnham, D. P.

DiLella, K. P. Lee, C. R. K. Marrian, and R. J. Colton, J. Vac. Sci.

R. Zhang and D. G. Ivey, J. Vac. Sci. Technol. B 14, 2 (1996).

J. A. Stroscio and W. J. Kaiser, Scanning Tunneling Microscopy (Academic Press, CA, 1993), Vol. 27, pp. 51-55.

G. S. Kelsy, J. Electrochem. Soc. 124, 818 (1977). 
J. C. Kotz and K. F. Purcell, Chemistry and Chemical Reactivity, 2nd ed., 711713 (1987)

M. S. Nikolova, A. Natarajan, P. C. Searson, G. S. Chakarova, and J. M. Macaulay, J. Electrochem. Soc. 144, 455-460 (1997).

H-W. Fink, IBM J. Res. Develop. 30, 460-462 (1986).

Linear Motion Vacuum Feedthrough, BLM-275-1, 2.75 Del Seal Flange, MDC High Vacuum Products, Hayward, CA.

RHK Technology, Inc., Rochester Hills, MI 48309.

Stepper Driver Card, Forthright Electronics, 2851 Mendecino Drive, Prescott Valley, AZ.

G. Binnig and D. P. E. Smith, Rev. Sci. Instrum. 57, 1688-1689 (1986).

R. G. Carr, J. Micr. 152, 379 (1988).

W. B. Powers, Piezo Film Sensing Devices (Soc. Manufact. Ingeneers., Michigan) 1986.

Roald K. Wangsness, Electromagnetic Fields (John Wiley \& Sons, N. Y., 1986), 2nd ed., pp. 338-342.

Hiroshi Kaizuka, Rev. Sci. Instrum. 60, 3119-3122 (1989).

P. J. Ouseph, Thomas Poothackanal, and George Mathew, Phys. Lett. A 205, 6571 (1995).

Sun M Paik, Sihong Kim, and Ivan Schuller, Phys. Rev. B 44, 3272-3276 (1991).

Naruo Sasaki and Masaru Tsukada, Phys. Rev. B 52, $8471-8482$ (1995). 
Sang-il Park, J. Nogami, H. A. Mizes, and C. F. Quate, Phys. Rev. B 38, 42694272 (1988).

T. R. Albrecht, H. A. Mizes, J. Nogami, Sang-il Park, and C. F. Quate, Appl.

Phys. Lett. 52, 362-364 (1988)

T. Klitsner, R. S. Becker, and J. S. Vickers, Phys. Rev. B 41, 3837-3840 (1990).

32

E. J. van Loenen, D. Dijkkamp, A. J. Hoeven, J. M. Lenssinck, and J. Dielman, Appl. Phys. Lett. 56, 1755-1757 (1990).

33

K. Hane and S. Okuma, J. Appl. Phys. 75, 1228-1230 (1994). 


\section{VITA}

The author, Michael Stephen Gossman, is the son of Stephen Kent Gossman and Diana Lynne Adams. He was born to his parents on the twelfth day of January 1972 in New Albany, Indiana. He was blessed in having Jennifer Lynne Gossman as a younger sister.

On the second day of June 1990, the author graduated from Jeffersonville High School with concentrations in electronics and aerospace. He attended general courses at Indiana University Southeast from 1990 to 1993 . As an outstanding undergraduate, the author was allowed a Technical/Teaching Assistantship in the Physics Department for two years there, under the direction of Chairman Dr. Kyle Forinash. In the year 1993, he transferred to the University of Louisville in pursuit of this collegiate diploma. Still eager to learn out of the classroom, he was given a Research Assistantship for six months, under Chairman Dr. Joseph Chalmers. The author graduated on the eighteenth day of August 1995 with a Minor Degree in Mathematics and a Bachelor of Science of Physics.

The author was soon accepted into the Graduate School at the University of Louisville. The Physics Department rewarded him for his experience and knowledge in the field by providing him with a Graduate Teaching Assistantship. In recognition of high scholarship and leadership in Physics, he was elected to be a member of the $\Sigma \Pi \Sigma$ National Physics Honor Society, inducted as Vice-President into the Louisville Chapter of the Society of Physics Students, and served on the Graduate Student Council from 1996 through 1997. He will continue his education at Vanderbilt University where he intends to pursue a degree in Medical Physics. 